\title{
Process Modeling of Impedance Characteristics of Proton Exchange Membrane Fuel Cells
}

by

Seyed Mohammad Rezaei Niya

B.Sc., Sharif University of Technology, Iran, 2002

M.Sc., Amirkabir University of Technology, Iran, 2005

A THESIS SUBMITTED IN PARTIAL FULFILLMENT OF

THE REQUIREMENTS FOR THE DEGREE OF

DOCTOR OF PHILOSOPHY

in

THE COLLEGE OF GRADUATE STUDIES

(Mechanical Engineering)

THE UNIVERSITY OF BRITISH COLUMBIA

(Okanagan)

May 2015

(C) Seyed Mohammad Rezaei Niya, 2015 


\section{Abstract}

The impedance characteristics of proton exchange membrane (PEM) fuel cells are studied and analyzed in this thesis. The modeling approaches presented in literature are thoroughly reviewed and categorized as the measurement-modeling and process-modeling approaches. In the former category, a hypothetical equivalent circuit which has the impedance characteristics similar to measured impedances is presented. Since the equivalent circuit is not directly resulted from the physical and chemical properties of the PEM fuel cells, the majority of the measurementmodeling approaches lead to dubious conclusions. In the process-modeling approach, on the other hand, the governing equations of the fuel cell must analytically be solved to determine and the impedance. However, a few process-modeling approaches presented in literature have shown to be indirectly based on the same assumptions as the measurement-modeling approach, and hence, those can also lead to similar conclusions. Therefore, these process-modeling approaches are referred to as the semi-process models here.

In this thesis, the first complete process model for PEM fuel cells is presented which is not based on the above-mentioned assumptions. For each source of the losses in the fuel cell (i.e., the ohmic, activation and concentration overpotentials), a process model and equivalent circuit are obtained and compared against the impedance measurements reported in literature. The complete model (obtained by combining the models of the three losses) is then verified against the impedances measured in different operating conditions. Using the verified model, the measured Nyquist plots of the PEM fuel cells reported in literature are categorized. As a result, the dominant physical and chemical parameters controlling various arcs of the Nyquist plot are determined. Finally, the sensitivity analysis of the impedance characteristics of fuel cells is conducted using the verified model. As a result of this analysis, a minimum change in the operating conditions which results in statistically different Nyquist plots are determined.

Finally, as an application of the model presented here, the impedance of the cell in the anode and cathode starvation modes are studied. It is shown that the anode starvation cannot be recognized from the impedance measurements, as predicted by the model. 


\section{Preface}

This research was conducted in the Advanced Thermo-Fluidic Laboratory (ATFL) at University of British Columbia, Okanagan campus, under supervision of Dr. Mina Hoorfar. The results of this research have been published in several peer-reviewed journal articles and conference proceedings.

Chapter 1 includes the discussions published as a review journal article (S.M. Rezaei Niya, M. Hoorfar (2013) 'Study of proton exchange membrane fuel cells using electrochemical impedance spectroscopy technique - A review', Journal of Power Sources, Vol. 240, pp. 281-293) and also a journal article discussing the details of the process modeling approach (S.M. Rezaei Niya, M. Hoorfar (2015) 'Measurement, semi-process and process modeling of proton exchange membrane fuel cells', International Journal of Hydrogen Energy, Vol. 40, pp. 4868-4873). I conducted all the studies and discussions and prepared the manuscripts.

A version of Chapter 2 has been accepted as a conference presentation (S.M. Rezaei Niya, R.K. Phillips, M. Hoorfar (2015) 'Improvement of the process model for the ohmic loss of the proton exchange membrane fuel cell', Accepted for 227 th ECS meeting, May 24-28, Chicago, USA) and submitted to ECS transactions (S.M. Rezaei Niya, R.K. Phillips, M. Hoorfar, 'Improvement of the process model for the ohmic loss of the proton exchange membrane fuel cell', Submitted to ECS Transactions). While Ryan Phillips assisted me in the design of the required fuel cell hardware and also conducting the impedance measuring processes, I designed the experiments, performed all the analytical analysis and required programming, and prepared the manuscripts. The model presented in this chapter is in fact the modified and improved version of the ohmic loss model published before as a journal article (S.M. Rezaei Niya, M. Hoorfar (2014) 'Process modeling of the ohmic loss in proton exchange membrane fuel cells', Electrochimica Acta, Vol. 120, pp. 193203) and also in a conference proceeding (S.M. Rezaei Niya, M. Hoorfar (2013) 'Impedance characteristics of the membrane of proton exchange membrane fuel cell', ASME $11^{\text {th }}$ fuel cell science, engineering \& technology conference (ESFuelCell2013), July 14-19, Minneapolis, USA). I also conducted analytical analysis and programming and prepared the manuscripts for these publications. 
A version of Chapter 3 has been published as a journal article (S.M. Rezaei Niya, M. Hoorfar (2015) 'Process modeling of electrodes in proton exchange membrane fuel cells', Journal of Electroanalytical Chemistry, Vol. 747, pp. 112-122). The details of the assumptions used in this chapter have been verified and published in two conference proceedings (S.M. Rezaei Niya, M. Hoorfar (2014) 'Determination of activation losses in proton exchange membrane fuel cells', ASME $12^{\text {th }}$ fuel cell science, engineering \& technology conference (ESFuelCell2014), June 29July 2, Boston, USA; and S.M. Rezaei Niya, M. Hoorfar (2014) 'Simplification of Butler-Volmer equation for proton exchange membrane fuel cell electrodes', CSME international congress, June 1-4, Toronto, Canada). I conducted the analytical analysis and prepared the manuscripts.

A version of Chapter 4 has been submitted as a journal article (S.M. Rezaei Niya, R.K. Phillips, M. Hoorfar 'Process modeling of the impedance characteristics of proton exchange membrane fuel cells', Submitted to Journal of Power Sources). While Ryan Phillips assisted me in conducting the experiments, I designed the experiments, performed all the analytical analysis and required programming, and prepared the manuscript.

A version of Chapter 5 has been submitted as a journal article (S.M. Rezaei Niya, R.K. Phillips, M. Hoorfar 'Sensitivity analysis of the impedance characteristics of proton exchange membrane fuel cells', Submitted to Journal of Power Sources). The sensitivity analysis method presented in this chapter is the extended version of the method presented and published as a paper in ECS transactions and three conference proceedings (S.M. Rezaei Niya, P.D. Barry, M. Hoorfar (2014) 'Sensitivity analysis of impedance characteristics of a laminar flow-based fuel cell', ECS Transactions, Vol. 58, pp. 49-58; S.M. Rezaei Niya, M. Hoorfar (2012) 'Temperature sensitivity analysis of electrochemical impedance spectroscopy results in PEM fuel cells', ASME $10^{\text {th }}$ fuel cell science, engineering \& technology conference (ESFuelCell2012), July 23-26, San Diego, California, USA; and S.M. Rezaei Niya, M. Hoorfar (2012) 'Dependency of measured impedance of PEM fuel cells to temperature variations', $19^{\text {th }}$ World Hydrogen Energy Conference (WHEC), June 3-7, Toronto, Canada; S.M. Rezaei Niya, P.D. Barry, M. Hoorfar (2013) 'Sensitivity analysis of impedance characteristics of a laminar flow-based fuel cell', 224 ${ }^{\text {th }}$ ECS meeting, Oct. 27-Nov. 1, San Francisco, USA). While Paul Barry assisted me in performing the impedance measurement of the laminar flow-based fuel cell, I designed the experiments, performed all the analytical analysis and required programming, and prepared the manuscript. 
A version of Chapter 6 has been submitted as a journal article (S.M. Rezaei Niya, R.K. Phillips, M. Hoorfar (2015) 'Study of anode and cathode starvation effects on the impedance characteristics of proton exchange membrane fuel cells', Submitted to Journal of Power Sources). While Ryan Phillips assisted me in conducting the experiments, I designed the experiments, performed all the analytical analysis and required programming, and prepared the manuscript.

Finally, a number of side-line projects have been conducted which are not covered in this thesis. The impedance characteristics of a laminar flow-based fuel cell were studied, and a measurement model was presented which was published as a journal article (S.M. Rezaei Niya, P.D. Barry, M. Hoorfar (2014) 'Study of crossover and depletion effects in laminar flow-based fuel cells using electrochemical impedance spectroscopy', International Journal of Hydrogen Energy, Vol. 39, pp. 12112-12119). The electron leakage in the membrane of the PEM fuel cell was studied, and the results were published in a transaction article (S.M. Rezaei Niya, R.K. Phillips, M. Hoorfar (2014), 'Estimation of leakage current in proton exchange membrane fuel cells', ECS Transactions, Vol. 61, pp. 33-38) and a conference proceeding (S.M. Rezaei Niya, R.K. Phillips, M. Hoorfar (2014) 'Estimation of leakage current in proton exchange membrane fuel cells', $225^{\text {th }}$ ECS meeting, May 11-16, Orlando, USA). The constant phase element was also studied and the results were accepted in a conference proceeding (S.M. Rezaei Niya, R.K. Phillips, M. Hoorfar (2015) 'Dependency of the power of the constant phase element to working conditions in proton exchange membrane fuel cells', Accepted for ASME $13^{\text {th }}$ fuel cell science, engineering \& technology conference, June 28-July 2, California, USA). 


\section{Table of Contents}

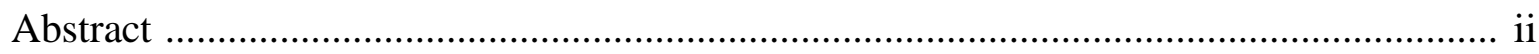

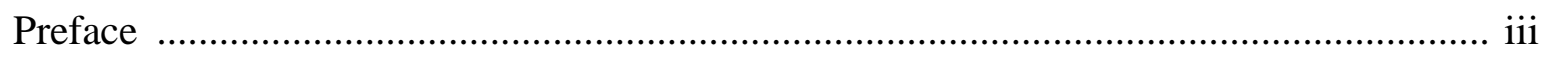

Table of Contents ...................................................................................................... vi

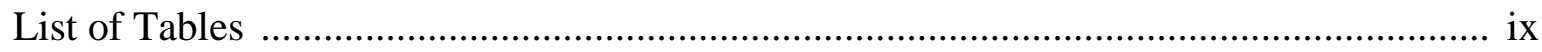

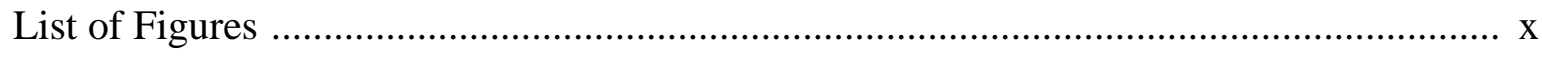

List of Symbols .............................................................................................. xiii

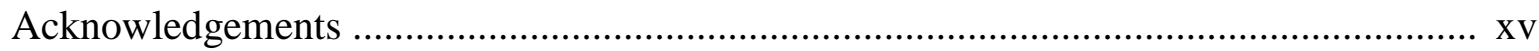

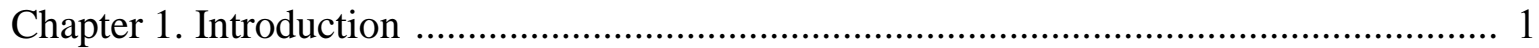

1.1 Applications of impedance spectroscopy in PEM fuel cell studies ................... 7

1.1.1 Temperature and humidity effects ................................................... 7

1.1.2 Sub-zero conditions ....................................................................... 8

1.1.3 Catalyst layer .............................................................................. 9

1.1.4 Gas diffusion and micro-porous layers ............................................ 10

1.1.5 Membrane ……………………………………………….... 12

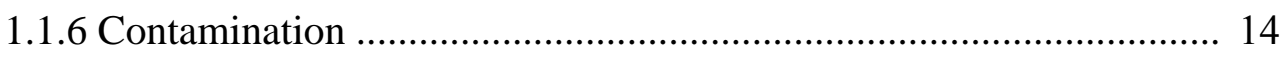

1.1.7 Aging and durability ................................................................... 16

1.1.8 Dehydration and flooding ……………………….......................... 16

1.1.9 Other phenomena diagnosed by EIS ............................................... 18

1.2 Measurement, semi-process and process modeling ……………………….... 19

1.2.1 Measurement modeling approach ……………………………….... 20

1.2.2 Process modeling approach ........................................................... 21

1.2.2.1 Procedure for obtaining the analytical solutions ............... 21

1.2.2.2 Importance of the equivalent circuit .................................. 22

1.2.2.3 Procedure for obtaining the equivalent circuit .................. 23

1.2.3 Semi-process modeling approach ................................................... 24

1.3 Motivations and objectives ...................................................................... 25

Chapter 2. Process model for ohmic loss .................................................................... 27

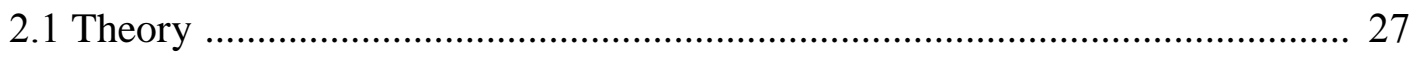

2.1.1 Water transport in the membrane .................................................. 30 
2.1.2 Water transport in the cathode catalyst and gas diffusion layers ..... 32

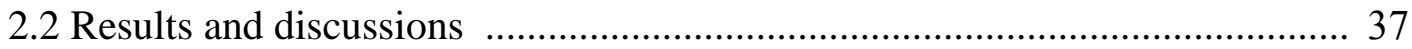

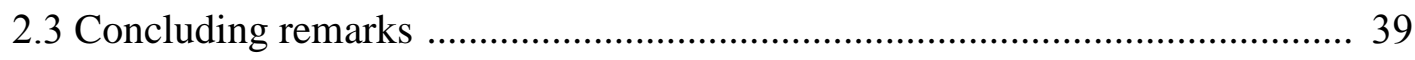

Chapter 3. Process modeling of electrodes ......................................................... 40

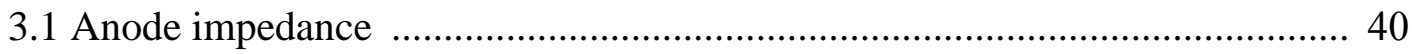

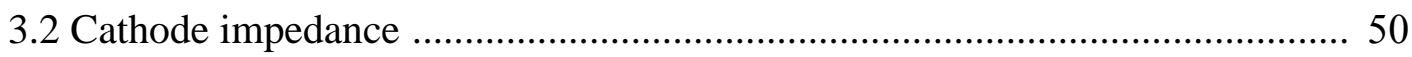

3.3 Validity of the Butler-Volmer equation estimations .................................. 56

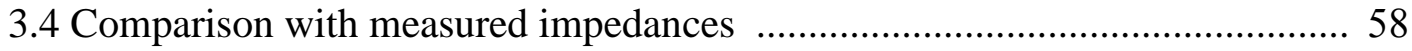

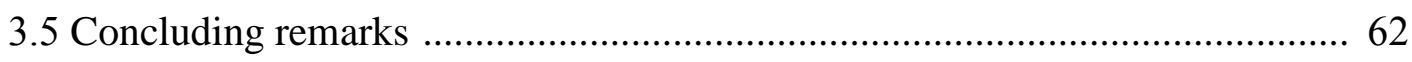

Chapter 4. Verification of the model f.................................................................... 63

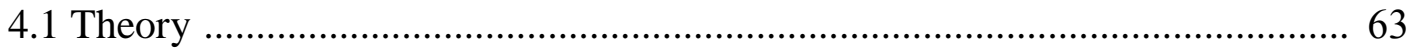

4.1.1 Activation and mass transport overpotentials ............................. 64

4.1.2 Ohmic overpotential ............................................................ 66

4.1.3 Overall impedance ............................................................ 67

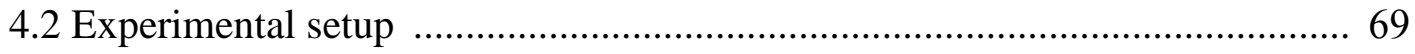

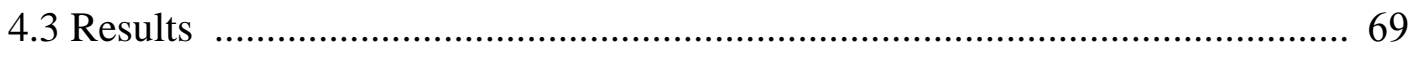

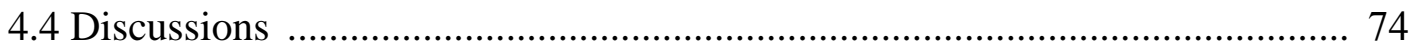

4.4.1 High frequency resistance ................................................ 75

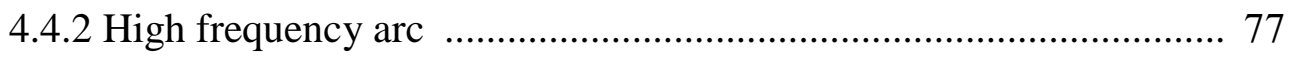

4.4.3 Mid-frequency arc ............................................................. 77

4.4.4 Low frequency arc ........................................................... 78

4.4.5 Inductive loop ................................................................... 80

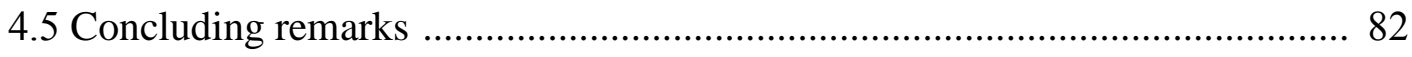

Chapter 5. Sensitivity analysis of the impedance characteristics ................................ 84

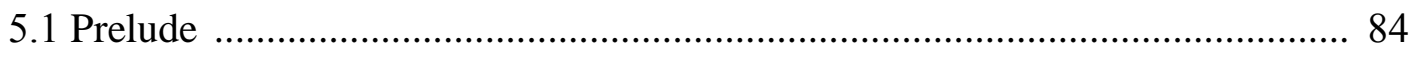

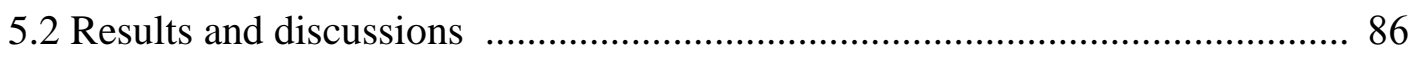

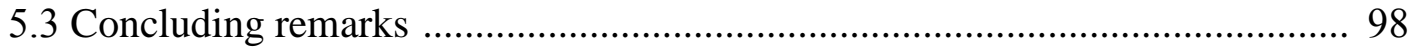

Chapter 6. Anode and cathode starvation effects ................................................. 100

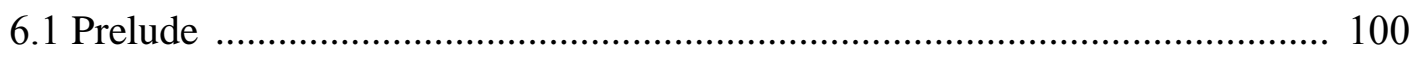

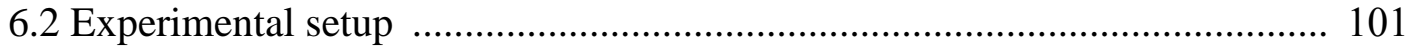

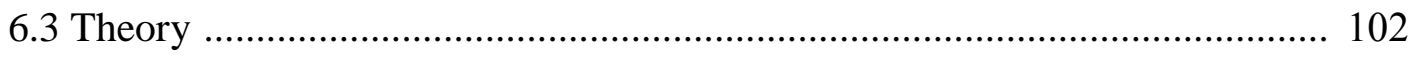

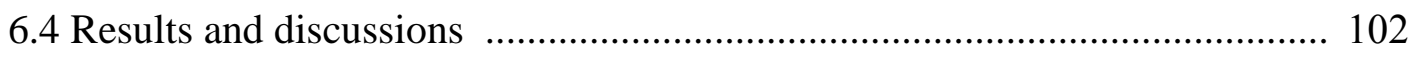

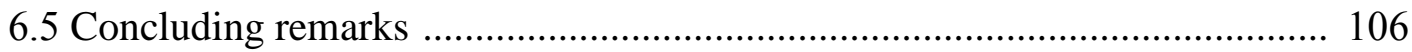


Chapter 7. Conclusions and future work .......................................................... 107

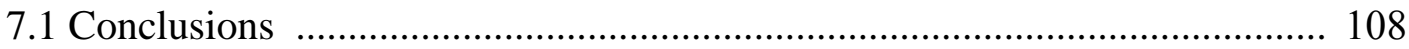

7.2 Proposed future work .................................................................. 108

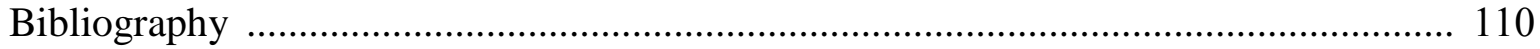




\section{List of Tables}

Table 3.1 Parameters used for calculating the elements of the anode equivalent circuit .. 48

Table 3.2 Parameters used for calculating the elements of the cathode equivalent circuit 54

Table 4.1 Values of parameters obtained from the best fit presented in Figure 4.4 ........ 70

Table 4.2 Physical dimensions of the cell and constants used in the model ................... 71

Table 4.3 The effect of different parameters on HFR ............................................. 76

Table 5.1 The calculated potential and current density indifference intervals ................ 90

Table 5.2 The calculated indifference intervals of the operating temperature and anode and cathode relative humidities ................................................................ 98 


\section{List of Figures}

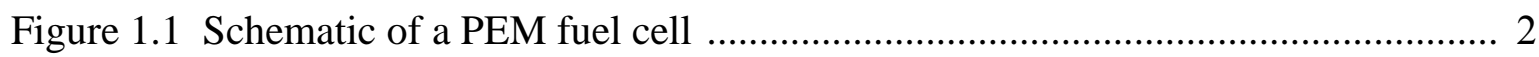

Figure 1.2 Randles equivalent circuit ......................................................................... 6

Figure 1.3 Conventional transmission line model ………………………................... 7

Figure 1.4 A typical Nyquist plot of a membrane ……………………………………. 13

Figure 2.1 The equivalent circuit of the ohmic loss …………................................. 36

Figure 2.2 Comparison between the curves obtained from the ohmic loss model and the measured impedances measured by Ref. [17] ............................................... 37

Figure 2.3 Comparison between the curves obtained from the analytical model and the measured impedances measured by Ref. [43] in the low frequency range .... 38

Figure 2.4 Comparison between the curves obtained from the analytical model and the measured impedances measured by Ref. [88] in the low frequency range .... 39

Figure 3.1 The schematic view of the anode-membrane interphase

Figure 3.2 The PEM fuel cell anode equivalent circuit; (a) the complete version, (b) the simplified version

Figure 3.3 The schematic view of the cathode-membrane interphase ………………..... 50

Figure 3.4 The PEM fuel cell cathode equivalent circuit ................................................ 53

Figure 3.5 (a) Comparison of the measured impedances presented by Malevich et al. [43] and the predicted impedances from the analytical solution; (b) The effect of the oxygen concentration correction is shown by asterisks

Figure 3.6 (a) Comparison of the measured impedances presented by Danzer and Hofer [17] and the predicted impedances from the analytical solution; (b) The effect of the oxygen concentration correction is shown by asterisks

Figure 3.7 Comparison of the measured impedances presented by Malevich et al. [31] and the predicted impedances from the analytical solution

Figure 4.1 The equivalent circuits for (a) anode (resulted from Equation (4.6)), and (b) cathode (Equation (4.7))

Figure 4.2 The equivalent circuit for the ohmic loss (Equation (4.10)) ........................... 67

Figure 4.3 The overall equivalent circuit of the PEM fuel cell

Figure 4.4 The measured and fitted Nyquist plots at $T=70^{\circ} \mathrm{C}, \mathrm{RH}_{A}=\mathrm{RH}_{C}=90 \%$, and $j=1.81 \mathrm{~A} \cdot \mathrm{cm}^{-2}$. The points corresponding to the frequencies of $10 \mathrm{~Hz}$ and $100 \mathrm{~Hz}$, are marked by ' +' and '* ', respectively

Figure 4.5 The measured and predicted impedances at (a) $T=65^{\circ} \mathrm{C}, \mathrm{RH}_{A}=\mathrm{RH}_{C}=90 \%$, and $j=2.11 \mathrm{~A} \cdot \mathrm{cm}^{-2}(0.7 \mathrm{~V})$; and (b) $T=75^{\circ} \mathrm{C}, \mathrm{RH}_{A}=\mathrm{RH}_{C}=90 \%$, and $j=2.02 \mathrm{~A} \cdot \mathrm{cm}^{-2}(0.7 \mathrm{~V})$. The points corresponding to the frequencies of $10 \mathrm{~Hz}$ 
and $100 \mathrm{~Hz}$, are marked by ' +' and ' *', respectively

Figure 4.6 The measured and predicted impedances at $T=70^{\circ} \mathrm{C}$ and $\mathrm{RH}_{A}=\mathrm{RH}_{C}=90 \%$.

The points corresponding to the frequencies of $10 \mathrm{~Hz}$ and $100 \mathrm{~Hz}$, are marked by ' + ' and ' $*$ ', respectively

Figure 4.7 The measured and predicted impedances at (a) $T=65^{\circ} \mathrm{C}, \mathrm{RH}_{A}=\mathrm{RH}_{C}=90 \%$, and $j=4.73 \mathrm{~A} \cdot \mathrm{cm}^{-2}(0.6 \mathrm{~V})$; (b) $T=75^{\circ} \mathrm{C}, \mathrm{RH}_{A}=\mathrm{RH}_{C}=90 \%$, and $j=4.54 \mathrm{~A} \cdot \mathrm{cm}^{-2}$ $(0.6 \mathrm{~V})$; (c) $T=70^{\circ} \mathrm{C}, \mathrm{RH}_{A}=90 \%, \mathrm{RH}_{C}=100 \%$, and $j=4.75 \mathrm{~A} \cdot \mathrm{cm}^{-2}(0.6 \mathrm{~V})$; and (d) $T=70^{\circ} \mathrm{C}, \mathrm{RH}_{A}=90 \%, \mathrm{RH}_{C}=80 \%$, and $j=4.38 \mathrm{~A} \cdot \mathrm{cm}^{-2}(0.6 \mathrm{~V})$. The points corresponding to the frequencies of $10 \mathrm{~Hz}$ and $100 \mathrm{~Hz}$, are marked by ' +' and ' $*$ ', respectively

Figure 4.8 The measured and predicted impedances at (a) $T=75^{\circ} \mathrm{C}, \mathrm{RH}_{A}=\mathrm{RH}_{C}=90 \%$, and $j=0.354 \mathrm{~A} \cdot \mathrm{cm}^{-2}(0.8 \mathrm{~V})$; (b) $T=70^{\circ} \mathrm{C}, \mathrm{RH}_{A}=90 \%, \mathrm{RH}_{C}=100 \%$ and $j=0.363 \mathrm{~A} \cdot \mathrm{cm}^{-2}(0.8 \mathrm{~V})$; (c) $T=70^{\circ} \mathrm{C}, \mathrm{RH}_{A}=80 \%, \mathrm{RH}_{C}=90 \%$, and $j=0.353 \mathrm{~A} \cdot \mathrm{cm}^{-2}(0.8 \mathrm{~V})$; and (d) $T=70^{\circ} \mathrm{C}, \mathrm{RH}_{A}=100 \%, \mathrm{RH}_{C}=90 \%$, and $j=0.339 \mathrm{~A} \cdot \mathrm{cm}^{-2}(0.8 \mathrm{~V})$. The points corresponding to the frequencies of $10 \mathrm{~Hz}$ and $100 \mathrm{~Hz}$, are marked by ' +' and ' * ', respectively

Figure 4.9 The schematic Nyquist plot of PEM fuel cell and the related blocks of the equivalent circuit obtained

Figure 4.10 The schematic of the MF arc variations in different current densities 78

Figure 4.11 The schematic of the Nyquist plots for the normal, dehydrated, and flooded modes

Figure 5.1 The polarization curves of the cell measured in different operating temperatures and anode and cathode relative humidities 86

Figure 5.2 The measured Nyquist plots in the temperature of $T=70{ }^{\circ} \mathrm{C}$, the anode relative humidity of $\mathrm{RH}_{A}=90 \%$, and the cathode relative humidity of $\mathrm{RH}_{C}=90 \%$. The error bars depict the deviations in three successive measurements. (a) Range of $0.85 \mathrm{~V}-0.7 \mathrm{~V}$; (b) Range of $0.7 \mathrm{~V}-0.55 \mathrm{~V} \ldots 87$

Figure 5.3 The calculated indifference intervals of the operating temperature $\left({ }^{\circ} \mathrm{C}\right)$ in different potentials; (a) $0.8 \mathrm{~V}$, (b) $0.7 \mathrm{~V}$, (c) $0.6 \mathrm{~V}$

Figure 5.4 The calculated indifference intervals of the anode relative humidity (\%) in different potentials; (a) $0.8 \mathrm{~V}$, (b) $0.7 \mathrm{~V}$, (c) $0.6 \mathrm{~V}$

Figure 5.5 The calculated indifference intervals of the cathode relative humidity (\%) in different potentials; (a) $0.8 \mathrm{~V}$, (b) $0.7 \mathrm{~V}$, (c) $0.6 \mathrm{~V}$ 96

Figure 6.1 The measured polarization curves of the cell fed with (a) the normal hydrogen and air stoichiometry ratio, (b) the mixture of $10 \%$ hydrogen and $90 \%$ helium in the anode and normal air in the cathode, and (c) the normal hydrogen stoichiometry ratio in the anode and the mixture of $2 \%$ oxygen and $98 \%$ nitrogen in the cathode

Figure 6.2 The measured and modeled Nyquist plots in the normal case. The points corresponding to the frequencies of $10 \mathrm{~Hz}$ and $100 \mathrm{~Hz}$, are marked by '+' and ' $*$ ', respectively 
Figure 6.3 The measured and modeled Nyquist plots in the starvation of the anode (the mixture of $10 \%$ hydrogen and $90 \%$ helium in the anode and the normal air stoichiometry ratio in the cathode) and the cathode (the normal hydrogen stoichiometry ratio in the anode and the mixture of $2 \%$ oxygen and $98 \%$ nitrogen in the cathode). Points corresponding to the frequencies of $10 \mathrm{~Hz}$ and $100 \mathrm{~Hz}$, are marked by '+' 'and '* ', respectively ............. 105

Figure 6.4 The comparison of the Nyquist plots in the normal and starved cases ....... 106 


\section{List of Symbols}

$C_{d l} \quad$ Magnitude of the double layer element

D Diffusion coefficient

$E_{\text {theor. }} \quad$ Theoretical potential

F Faraday's constant

$J \quad$ Water flux

$M_{\mathrm{M}} \quad$ Membrane equivalent weight

$R \quad$ Ideal gas constant

RH Relative humidity

$T \quad$ Temperature

$V \quad$ Fuel cell potential

Z Impedance

$Z_{\text {act. }} \quad$ Impedance of the activation overpotential

$Z_{\text {ohm. }} \quad$ Impedance of the ohmic overpotential

$Z_{\text {tot }} \quad$ Overall impedance

$Z_{\text {trans. }} \quad$ Impedance of the mass transport overpotential

$a \quad$ Defined parameter $(=0.5193 \exp (1268(1 / 303-1 / T))$

$b \quad$ Defined parameter $(=0.326 \exp (1268(1 / 303-1 / T))$

c Concentration

$c_{\mathrm{H}^{+}, \mathrm{s}} \quad$ Actual adsorbed proton concentration

$c_{\mathrm{H}_{2}, \mathrm{~s}} \quad$ Concentration of the adsorbed hydrogen gas

$c_{\mathrm{O}, \mathrm{s}} \quad$ Actual adsorbed oxygen atom concentration

$d \quad$ Double-layer thickness

$f \quad$ Flooding coefficient

$i \quad$ Imaginary unit $(\sqrt{-1})$

j Current density

$j_{\mathrm{H}^{+}} \quad$ Proton-transfer's current density 


$\begin{array}{ll}j_{0} & \text { Exchange current density } \\ l & \text { Thickness } \\ n & \text { Number of electrons } \\ n_{\mathrm{CPE}} & \text { Power of the constant phase element } \\ t & \text { Time } \\ \Gamma_{\mathrm{H}^{+}} & \text {Surface density of the protons } \\ \alpha & \text { Defined parameter }(=2.5 /(22 F)) \\ \alpha_{\mathrm{C}} & \text { Cathode transfer coefficient } \\ \beta & \text { Defined parameter }\left(=\rho_{\mathrm{M}} D_{\mathrm{M}} / M_{\mathrm{M}}\right) \\ \varepsilon_{s} & \text { Dielectric permittivity } \\ \eta & \text { Overpotential } \\ \eta_{c h} & \text { Channel efficiency } \\ \lambda & \text { Water content } \\ \rho_{\mathrm{M}} & \text { Membrane dry density } \\ \sigma & \text { Electrical Conductivity } \\ \omega & \text { Angular velocity } \\ ()^{\mathrm{w}} & \text { Water } \\ ()^{0} & \text { Reference value } \\ ()_{\mathrm{C}} & \text { Value prior to perturbation } \\ ()_{A} & \text { Anode } \\ ()_{C} & \text { Cathode } \\ ()_{\mathrm{CL}} & \text { Catalyst layer } \\ ()_{\mathrm{GDL}} & \text { Gas diffusion layer } \\ \eta^{*} & \end{array}$




\section{Acknowledgements}

My deepest gratitude goes to my dear supervisor, Dr. Mina Hoorfar, who helped and supported me during my $\mathrm{PhD}$ career at University of British Columbia. I want to deeply thank her for all her support, trust, passion and time. I had this privilege to work under the supervision of a passionate researcher and devoted teacher who helped me to acquire invaluable experiences.

I would also like to thank my thesis committee members, Dr. Homayoun Najjaran, Dr. Thomas Johnson, and Dr. Wilson Eberle for their insightful comments, suggestions and ideas.

I would like to thank Ryan Phillips for his help in conducting the experimental measuring during the study. Also, I would like to thank Paul Barry for his contribution in the experimental measurements.

Finally, I want to thank my smart, beautiful and lovely wife, Serveh, for all her support and love. 


\section{Chapter 1. Introduction ${ }^{1}$}

Global warming, environmental pollutions and oil crisis are just examples of large-scale phenomena directly related to conventional power generation systems and fossil fuels. There are a prodigious number of studies conducted on the development of innovative, inexpensive, clean and robust power generation systems to replace the current conventional systems. Among different alternative systems, fuel cells have shown a great potential to be considered as an alternative solution to the problems of current systems. Fuel cells have considerably high power densities and virtually no emission. Moreover, fuel cells do not have any moving parts, increasing their reliability and robustness for the long-term usage. In comparison to batteries, fuel cells have independent power (i.e., proportional to the fuel cell size) and capacitance (i.e., proportional to the reservoir size) characteristics which are important in a wide range of applications. Unlike batteries, fuel cells can also be recharged quickly by refuelling [1, 2].

Like any other systems, fuel cells also have limitations. The final cost of fuel cells compared to other systems is high. Hydrogen, which is the best fuel for low temperature fuel cells, has low volumetric energy density, and as a result, it is hard to store. Moreover, hydrogen has to have a very high-purity grade (especially in proton exchange membrane (PEM) fuel cells), and alternative fuels need reforming [2]. In addition, the robustness and durability of the fuel cells in start-stop cycles are questionable. The US department of energy (DOE) plan is to design a portable power fuel cell system $(<250 \mathrm{~W})$ with an energy density of $900 \mathrm{WhL}^{-1}$ by 2015 , a transportation fuel cell system with 5,000-hour durability by 2017, and a micro-CHP (Combined Heat and Power) fuel cell system (5 kW) with 60,000-hour durability and a medium-scale CHP fuel cell system (100 kW - 3 MW) with 80,000-hour durability by 2020 [3].

\footnotetext{
${ }^{1}$ Parts of Chapter 1 have been published in two journal articles. Reprinted with permission from [1, 202].
} 


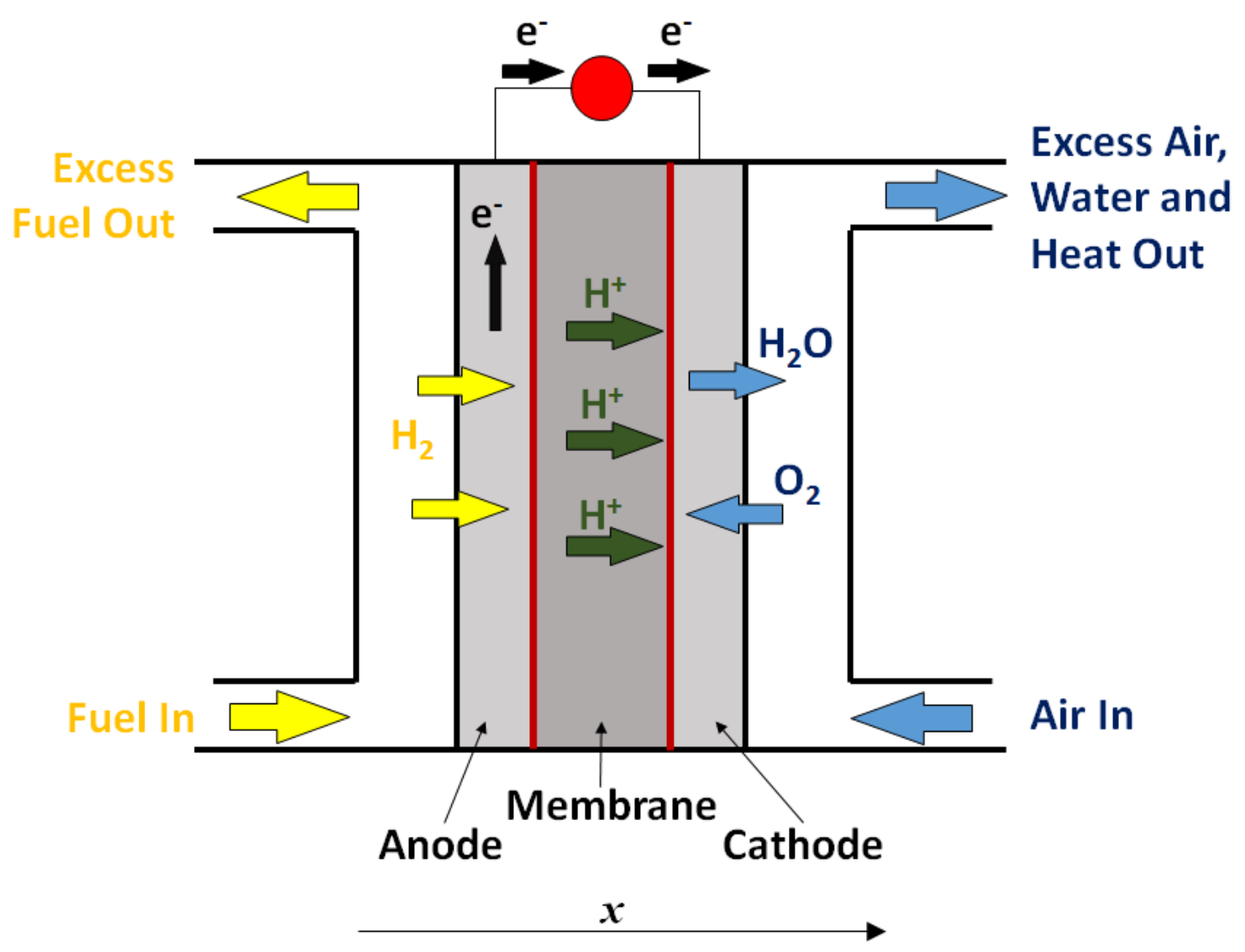

Figure 1.1 Schematic of a PEM fuel cell

Among various types of fuel cells, a proton exchange membrane (PEM) fuel cell has drawn much attention because of its low operating temperature and relatively high power density. The schematic of a PEM fuel cell is shown in Figure 1.1. In this system, the pure hydrogen is fed into the anode channels as fuel; while usually the air is fed into the cathode channels. The reactant gasses flow through the flow channels which can be in the form of parallel, serpentine, interdigitated or any innovative structures [e.g., 4]. Through diffusion and convection, the reactants travel through the gas diffusion layer (GDL) placed over the channels. The other side of the GDL is coated with a very thin layer of platinum making the catalyst layer (CL) at which the main electrochemical reactions occur. The reactions include the hydrogen oxidation reaction (HOR) in the anode and the oxygen reduction reaction (ORR) in the cathode, respectively [2].

$$
\mathrm{H}_{2} \rightarrow 2 \mathrm{H}^{+}+2 \mathrm{e}^{-}
$$




$$
\frac{1}{2} \mathrm{O}_{2}+2 \mathrm{H}^{+}+2 \mathrm{e}^{-} \rightarrow \mathrm{H}_{2} \mathrm{O}
$$

Water and heat are the by-products of these reactions. While the heat can be transferred and controlled by cooling the fuel cell, water management is a critical task in the fuel cell performance $[5,6]$. The gas diffusion layer (GDL) in the cathode side plays a vital role in transferring the excess water to the cathode channels as well as supplying the oxygen to the cathode catalyst layer.

As it is shown in Figure 1.1, the electrons transport via an external electrical connection; however, the $\mathrm{H}^{+}$ions (protons) must be transferred via the membrane. To facilitate proton transfer from the anode to the cathode, different materials have been presented as the membrane in different types of fuel cells. In proton exchange membrane (PEM) fuel cells, the persulfonated polytetrafluoroethylene (called as Nafion $®$ ) membrane is widely used. The ionic conductivity of Nafion changes linearly with its water content [2]. Thus, the membrane must be very well hydrated for proton transfer across the Nafion structure. The membrane dehydration happens when there is not enough water in the membrane. On the other hand, having excess water in the fuel cell network blocks the oxygen passages through the cathode gas diffusion layer: this phenomenon is referred to as flooding [2]. Therefore, there is a fine water balance that must be managed through the cell operation. However, finding and maintaining this balance is not a trivial task as it depends on many parameters (like the gas and cell temperature, gas humidity, flow channel geometry, GDL morphology, etc.).

There are different electrochemical methods developed to study and diagnose the performance of PEM fuel cells, especially for water management. These methods have been thoroughly reviewed by $\mathrm{Wu}$ et al. [7]. Among different techniques used, the polarization curve, current interruption, cyclic voltammetry and electrochemical impedance spectroscopy (EIS) are the most well-known electrochemical techniques in the fuel cell studies. The polarization curve is the most common method of presenting and discussing the PEM fuel cell performance [2, 7, 8]. In this technique, the cell potential is plotted against the cell current (or current density) in various operating conditions. Usually, the plot is divided into three (or more) parts. It is believed that each part presents a dominant performance loss. In the current interruption technique, the ohmic load is almost immediately released from the fuel cell and the potential change is plotted versus time. This method is often employed to investigate the ohmic losses in fuel cells [2, 7]. In the cyclic voltammetry technique, the potential increases linearly and sweeps back while the current 
is recorded. The resultant voltage-current plot has peaks in the potentials related to the electrochemical reactions of the system $[2,7]$.

Electrochemical impedance spectroscopy (EIS) is a well-established diagnostic and modeling method that has widely been used in electrochemistry [9-11] due to its flexibility (which enables covering a wide range of aspects of fuel cells) and accuracy. In this method, a harmonic perturbation is imposed to the system and the impedance of the system is measured in a wide range of frequencies. For this measurement, a frequency response analyzer (FRA) and a load bank are required. A sinusoidal wave is produced by the FRA and applied to the fuel cell via the load bank. Then, the system response is captured by FRA, and an impedance spectrum is calculated [8]. The measurements can be conducted in the galvanostatic or potentiostatic modes, perturbing the current or voltage by a harmonic wave, respectively. It has been reported that there is no considerable difference between the results obtained from the galvanostatic and potentiostatic modes [8]. The impedance measurement can also be conducted in both in-situ and ex-situ manners [8]. In the in-situ configuration, which has been widely used, the AC impedance of the fuel cell is measured as a whole; whereas, in the ex-situ measurement different components of the fuel cell are studied separately.

One of the most important parameters in the AC impedance measurement is the AC amplitude. It is clear that the larger the AC amplitudes the easier the separation of the measured response from noise. However, the amplitude is limited due to the linearity assumption: in essence, the impedance is estimated by measuring the changes in the potential over the changes in the current. This calculation can be accepted if the potential changes linearly with respect to the current. Since this is not true for PEM fuel cells and other electrochemical systems, the signal amplitude has to be small enough to satisfy the linearity assumption. Different studies [12-15] have indicated the AC signal amplitude equal to $5 \%$ of the DC performance current. For instance, the study conducted by Dale et al. [15] on the impedance spectra of a 2-kW stack for the selected AC amplitudes of 1\%, 5\%,7.5\%, 10\% and 15\% of the DC load shows that i) the spectrum is very scattered for low load currents, especially at a $1 \%$ AC signal amplitude for which it becomes difficult for FRA to distinguish between the response and noise; and ii) there is no considerable difference between the impedance plots in the amplitude range of $5 \%$ to $10 \%$.

The connecting wires can create significant magnetic fields affecting the impedance spectrum measurements [15]. This effect can be controlled and minimized by i) spirally winding the 
connecting wires, ii) making perfect connections, and iii) using equal length coaxial cables to connect to FRA. The inductive effect of the wires on the impedance spectra at high and intermediate frequencies has been frequently reported in the past $[15,16]$. In fact, it has become a trend in the literature to interpret the positive inductive impedances at high frequencies as the inductive effects of wires. Attempts have also been made [17] in modeling this effect by resistorinductor parallel connected elements in the equivalent circuit for high frequencies. Also, it is possible to use an open cell (as a calibration tool) to subtract the wires-related high frequency inductance effects from the measurements [18].

After measuring the fuel cell impedance in a certain frequency range, the data are plotted in the form of the Nyquist (the imaginary part of the impedance vs. the real part), the Bode phase (the impedance phase vs. frequency) or the Bode magnitude (the impedance magnitude vs. frequency) plots and then modeled using the measurement or process model approaches. In the measurement modeling approach, an equivalent circuit is presented by comparing the impedance characteristics of the circuit with those of the fuel cell measured and presented in the Nyquist (or Bode) plot. Despite the extensive use of this modeling approach, the elements of the equivalent circuits are not directly related to any physical properties of the cell, and hence no physicochemical conclusions can be presented based on the measurement-model equivalent circuits [1,9]. Furthermore, the presented equivalent circuits can be employed just in the neighborhood of the measured performance points and cannot be extended to other operating conditions (e.g., current densities, flow rates, humidity levels, etc.) [9, 19, 20]. The process models, on the other hand, are based on physical and chemical characteristics of the system. In these models, the governing equations are derived and analyzed, and the impedance characteristics of the system are determined. Then, the equivalent circuit is specified based on the impedance characteristics and the predicted impedance plots are compared against the measured values [9]. These two categories along with the new category clarified in this thesis (semi-process modeling approach) are discussed thoroughly in Section 1.3.

The most common equivalent circuit used in the measurement models is the Randles circuit. This circuit, which is originally proposed by Randles [21, 22], has been obtained based on the reaction of a metal ion in an aqueous solution using the process model. The original Randles circuit includes a frequency-dependent resistor and capacitor, which are parallel to a conventional capacitor, and a conventional resistor which is in series with the entire system. The Warburg element was introduced to the original circuit for the first time by Grahame [23]. He 
divided the electrochemical reactions into six different classes and presented various equivalent circuits. He presented the frequency-dependent characterizations of the resistor and capacitor which come from the linear diffusion equation in an anomalistic electrical element called Warburg. Figure 1.2 shows the Randles circuit including the Warburg element. The Randles equivalent circuit has not been determined based on the fuel cell governing equations. However, by replacing the conventional capacitor with another electrochemistry-based element, called the constant phase element (CPE), this circuit can capture majority of impedance measurements of the PEM fuel cell as shown in Ref. [24].

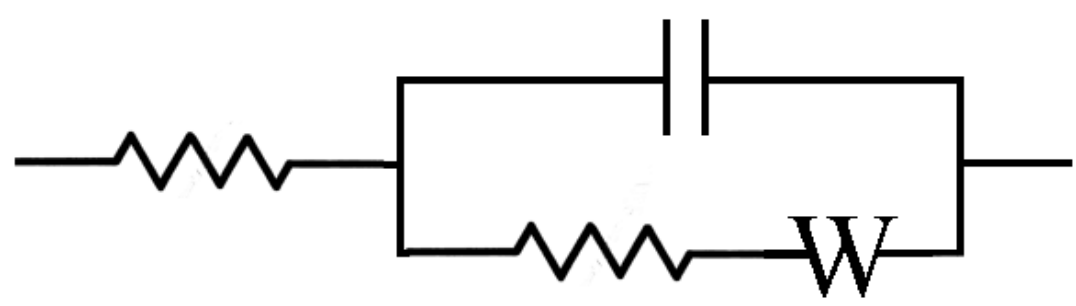

Figure 1.2 Randles equivalent circuit

There are modifications to the Randles equivalent circuit that can improve the predicted results. As mentioned above, the double layer capacitance can be replaced by a constant phase element (CPE) originated from the non-uniform distribution of the current density along the electrode surface as a result of surface non-homogeneity [10]. Considering the capacitor impedance as $Z=1 /(\mathrm{Ci} \omega)$, where $\omega$ is the angular frequency and $i$ is the imaginary unit, the impedance of a CPE element is defined as

$Z_{C P E}=\frac{1}{Q(i \omega)^{n}}$

where $n$ is called the CPE power and depends on physical properties $(0<n<1)$ and $Q$ represents the double layer capacitance. The CPE power is expected to remain constant during a wide range of operating conditions; otherwise, it will be an extra degree of freedom (in the measurement models) reducing the difference between the fitted curve and the measured impedances [24].

Another modification is the implementation of the transmission line model (TLM) to study the catalyst layer as porous media [25-30]. The model has been presented to capture the variations of 
ionic resistances inside the electrodes [27, 31], and assumes a network of cylindrical pores with uniform diameters in the catalyst layer [28]. In this method, the fuel cell (or a part of the cell) is modeled by a combination of electrical blocks. Each block contains normally a resistor-capacitor combination (Figure 1.3). It has been claimed that this equivalent circuit represents the governing equations when the number of blocks approaches to infinity [29, 32]. However, there are still debates regarding the consistency of the TLM with experimental results obtained at low relative humidity [25].

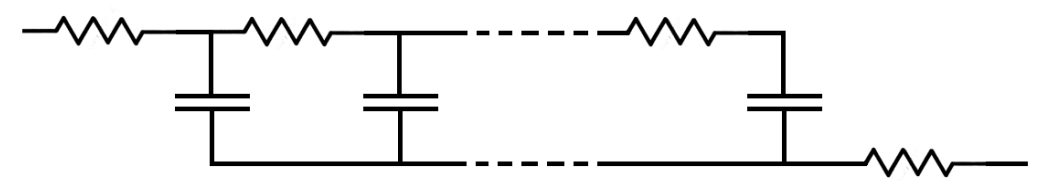

Figure 1.3 Conventional transmission line model (TLM)

\subsection{Applications of impedance spectroscopy in PEM fuel cell studies}

Electrochemical impedance spectroscopy (EIS) technique has been used widely to study and diagnose the PEM fuel cells. These studies are reviewed in the following sections:

\subsubsection{Temperature and humidity effects}

There is a wide range of studies conducted on the effect of temperature, relative humidity and gas flow rates on the fuel cell performance. Aaron et al. [33] studied the effects of temperature, air-side humidity and air flow rate on the performance of fuel cells using the EIS technique. The experiments have been performed in the temperature range of $30^{\circ} \mathrm{C}$ to $50^{\circ} \mathrm{C}$, the humidity range of $20 \%$ to $98 \%$ and the air stoichiometric ratios of 1.33 to 2.67 . They concluded that the air-side flow rate does not affect the impedance plots considerably as long as it is maintained at stoichiometric ratios greater than 1.2. However, the reproducibility of their results is debatable. They stated that the response in the second measurements showed the same trend, but the values were approximately $8 \%$ larger than those obtained in the first measurement.

Malevich et al. [34] conducted research on the effect of relative humidity on the active area in the impedance plots. They compared the Nyquist plots of $\mathrm{H}_{2} / \mathrm{H}_{2}, \mathrm{H}_{2} /$ Air, $\mathrm{H}_{2} / \mathrm{O}_{2}$ and $\mathrm{H}_{2} / \mathrm{N}_{2}$ 
feedings and then presented the physical/chemical interpretations for various parts of the Nyquist plot. They reported that decreasing the relative humidity of reactant streams increases the membrane resistance and reduces the active area. They suggested that the change in charge transfer resistance of the cathode because of relative humidity is due to the change in the active area. Their research has been extended to the study of the effects of relative humidity on membrane and catalyst layer resistances and microporous layer influences [35]. They concluded that the presence of micro porous layer in each of electrodes can have considerable effects on water management and humidity profile.

Danzer and Hofer [20] used EIS to study the dew point temperature in the humidifier, the excess ratio of hydrogen and oxygen and different operating points. They discussed about different equivalent circuits and found the equivalent circuit parameters via a nonlinear least square method. They stated that the ohmic resistance in constant humidification was not affected by current densities. In essence, the increase in the relative humidity shifts the impedance plot solely toward lower ohmic resistances due to the increase in the water content of the membrane.

Brunetto et al. [36] studied different relative humidity and flow rates using EIS and found the optimum stoichiometric coefficients. They designed their own hardware and software to measure the impedance of the fuel cell and presented empirical correlations for the electrolyte resistance based on the impedance results. Their measurements and calculations indicated that among different stoichiometric (ST) ratios the ST values of 3 and 4, for hydrogen and air respectively, produce a relatively high performance with low reactant fluxes (which results in a lower cost).

\subsubsection{Sub-zero conditions}

There are studies in the literature discussing about the fuel cell performance in sub-zero conditions. Hou et al. [37] measured the ionic resistance of the catalyst layer at four different current densities after eight freezing/thawing cycles. They reported that the catalyst layer ionic resistance decreases after cycles and the changes are not uniform. They studied the cold start process in sub-zero conditions in their subsequent paper [38] and concluded that the thin water film can cover pores among agglomerates and block the reactant gas. This topic was also studied by other research groups [39].

Mukandan et al. [40] studied the MEA (membrane electrode assembly) effect in the PEM fuel cell performance at sub-zero condition. They reported that the rate of the voltage decrease in the 
sub-freezing environment is because of the increment of the charge transfer resistance. Furthermore, they found that MEA is the dominant factor in ice formation in the catalyst layer, and the ice formation is more close to the cell edges and flow field turns.

\subsubsection{Catalyst layer}

Nitta et al. [41] evaluated the contact resistance between the gas diffusion layer and catalyst layer as a function of the compression using EIS. They claimed that this resistance is a considerable part of the total ohmic resistance of MEA and can severely affect the current distribution inside the cell. They estimated this resistance from the intersection of the high frequency imaginary impedance in the Nyquist plot with the real axis which is the only extracted impedance data that they employed in their paper.

Eastcott and Easton [42] studied a catalyst layer without sulfonic acid groups. They used cyclic voltammetry and electrochemical impedance spectroscopy to investigate the effect of $\mathrm{SiO}_{2}$ loading on the catalyst layer conductivity and active area. They concluded that $45 \% \mathrm{SiO}_{2}$ loading maximizes the active area although the fuel cell performance was lower than that achieved with the Nafion-based catalyst layers. In their subsequent paper [43], they studied the ceramic carbon electrodes and claimed it has similar performance to the Nafion-based electrodes.

Seo et al. [44] estimated the interfacial resistance between the membrane and catalyst layer by feeding $\mathrm{H}_{2} / \mathrm{H}_{2}$ to the fuel cell and then measuring the frequency-dependent impedance. Their presented equivalent circuit is an arbitrary measurement model which can be employed to model the measured impedances; however, this model is not based on any physical/chemical analysis, and hence the presented conclusions from the impedance measurements have not been verified. Cimenti et al. [25] used EIS to measure the catalyst layer proton resistance using $\mathrm{H}_{2} / \mathrm{N}_{2}$ and $\mathrm{H}_{2} / \mathrm{O}_{2}$ feeding methods. They modeled the catalyst layer with a transmission line model (TLM) and employed ZView software to fit the model to the measured impedances. They stated that TLM does not produce a good fit for dry conditions. They also found that the $\mathrm{H}_{2} / \mathrm{N}_{2}$ feeding method is capable of measuring the catalyst layer resistance at equilibrium and extracting microstructural information. This method is also preferred due to its low measuring time and simple data analysis. They also used this method to study the catalyst layer resistance dependency to relative humidity and concluded that decreasing the relative humidity increases the catalyst layer resistance in an exponential trend. 
Manzo et al. [45] studied the cathode catalyst layer and presented a model based on diffusion and current generation equations. Using the equivalent circuit proposed in [46], the anode and contact impedances were separated from the fuel cell impedance plot, and the cathode impedance was compared to the simulated results generated based on the governing equations. This separation of the fuel cell impedances into the anode, cathode and contact impedances is based on the assumption that the origin of the high frequency semicircle in the Nyquist plot is from the anode impedance. Using the same methodology, the same group studied the lowcurrent electrochemical mechanisms of the fuel cell [47] based on the assumption that mass transport limitations are negligible in low current densities. In both of their papers [45, 47], the theoretical formulations were transferred to the time domain, and spatial and temporal current distributions were calculated and discussed. Recently, Kulikovsky and Eikerling [48] presented a physical model for impedance of the cathode catalyst layer which took into account the voltage loss due to proton transport in the cathode catalyst layer. They claimed the Tafel slope, double layer capacitance, and cathode catalyst layer ionic conductivity can be determined by fitting the measured impedances to their model.

There is a group of published studies in literature regarding the catalyst layer studies which employed EIS besides other methods such as polarization curves, cyclic voltammetry (CV), transmission electron microscopy (TEM), scanning electron microscopy (SEM), X-ray diffraction (XRD), and $\mathrm{N}_{2}$ adsorption-desorption [49-58]. In all of these studies, the EIS is used to verify the results obtained from other methods rather than specifically discussing the measured impedances. Moreover, these studies often used simplifications in their EIS theory, and hence their discussions and the conclusions based on their assumptions are often questioned in literature $[8,9]$.

\subsubsection{Gas diffusion and micro-porous layers}

The EIS technique has also been used to study the effect of the gas diffusion layer on the cell performance. Bultel et al. [59] studied the effects of slow diffusion of oxygen in the gas diffusion layer (GDL) on the PEM fuel cell behaviour at various cell temperatures and relative humidities. They fed the cathode with a depleted oxidant concentration and also with different inert gases and concluded that the oxygen diffusion is a major concern and the oxygen diffusion coefficient in the gas diffusion layer is a governing parameter which can affect the fuel cell performance independent of the oxygen concentration. In the recent work conducted by Maidhily et al. [60], 
the GDL has been studied by measuring the impedances of the cathode and anode separately using $\mathrm{H}_{2} / \mathrm{H}_{2}$ and air/air feedings. Physical interpretations about different elements in the equivalent circuit were presented. However, the reported physical and chemical interpretations can be questioned due to the simplified assumptions employed. Another study conducted on the GDL using EIS is the work of Dotelli et al. [61] who used the combination of the polarization curves and impedance measurements to clarify the role of the GDL. In their study, where three different GDLs were used, five different impedance measurements have been obtained in each tested current densities, and the average values were presented as the final impedance spectra. They reported that the main difference between the tested GDLs is their contact resistances. They also confirmed that the presence of the micro porous layer (MPL) improves the performance of the GDL, especially at high current densities, mainly due to decreasing the contact resistance. They also claimed that increasing the compression reduces the fuel cell performance at high current densities.

Omati et al. [62] studied four different GDLs and their role in the fuel cell performance using both polarization curves and EIS. They presented a simple equivalent circuit and calculated different resistances based on the circuit. They reported that the GDL with the smallest thickness and weight produces the best performance. The same research group prepared a multi-wall carbon-nanotube-based gas diffusion medium and tested it using the fuel cell performance measurements and impedance characteristics [63]. Similar equivalent circuit was used. They found that adding carbon nanotubes increases the fuel cell performance. In their proposed equivalent circuit, the cell impedance has been divided into two parts showing the effect of the charge and mass transfer limitations; however, this conclusion has not been proven completely in literature.

The effects of the MPL on the performance and impedance behaviour of the fuel cell have also been studied by Malevich et al. [46]. The ohmic, charge and mass transfer resistances at different current densities were extracted from the impedance plots. They reported that the MPL decreases the mass transfer resistance without changing the charge transfer resistance, decreases the through-plane gas permeability of the porous layer, reduces water saturation in the porous layer, improves oxygen transport to the cathode catalyst layer, and consequently increases the fuel cell performance. In their subsequent papers [26, 30], the same research group measured the catalyst layer resistance and effective protonic conductivity by feeding $\mathrm{H}_{2} / \mathrm{N}_{2}$. They found that the assumption of a homogenous structure for the catalyst layer as well as modeling the catalyst 
layer with the uniform resistance and capacitance in the equivalent circuit are not accurate. They employed transmission line models (TLM) to capture the catalyst layer non-homogeneities which can be due to relative humidity in the measurement [64], the catalyst layer preparation method [64], and the level of ionomer impregnation in the catalyst layer [65].

Other studies on the effect of the GDL and MPL using EIS [66-69] have been based on arbitrary equivalent circuits. These circuits have been used to model the impedance plots and to explain the physicochemical properties. However, the presented equivalent circuits must be recognized as measurement models which can only be used to compare the impedances in different conditions and no physicochemical interpretation and conclusions can be presented based on the element values.

\subsubsection{Membrane}

The EIS technique has also been used to study the ionic conductivity of the membrane. The membrane ionic conductivity measurement using two-probe and four-probe techniques was compared by Mikhailenko et al. [70]. In two-probe technique, the response of the system is measured via the same electrodes to which the perturbation is applied. In the four-probe technique, on the other hand, measuring the system response and applying the perturbation to the system are achieved via two different pairs of electrodes [71]. They concluded that the twoprobe technique is less complicated, has a simpler geometry, and is less prone to the experimental errors. Also, it has been shown that the cell geometry and electrode arrangement has considerable effects on the frequency-dependent response. The ionic conductivity measurement has also been discussed by other groups [72], and it was shown that the ionic conductivity of their experimental membrane is similar to that of Nafion.

Soboleva et al. [18] determined the conductivity of various Nafion membranes by measuring the impedances in two perpendicular directions. To eliminate the inductive effects of the connecting wires in high frequencies, the impedance of the "opened cell" was measured and subtracted from the subsequent measurements. The typical Nyquist plot of a membrane is shown in Figure 1.4. To extract the through-plane membrane resistance from the Nyquist plot, two different methods in the literature are employed [18]. In the first method, the resistance is determined by extrapolating the linear portion of the low frequency part of the Nyquist plot to the real part axis ( $x$-axis). This point is assumed to be the through-plane resistance. In the second method that seems to be more accurate, however, the high frequency part of the impedance is modeled with a 
parallel combination of a resistor and a capacitor. The resistance is assumed to be the throughplane membrane resistance. Soboleva et al. [18] calculated the resistance via both methods and concluded that the deviations are less than $10 \%$.

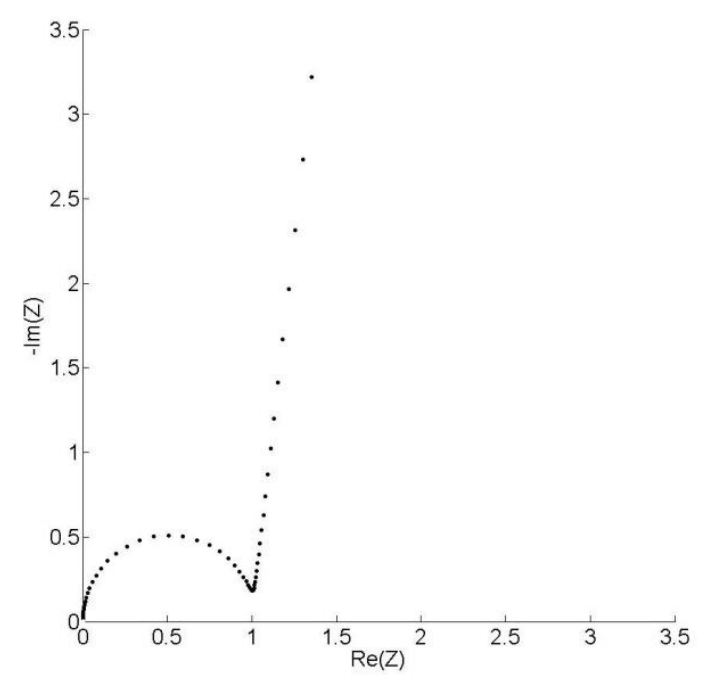

Figure 1.4 A typical Nyquist plot of a membrane

Liu et al. [29, 73] employed EIS to quantify the through-plane RH-dependent membrane resistance for various ionomer/carbon weight ratios and various operating conditions using $\mathrm{H}_{2} / \mathrm{N}_{2}$ feeding. They reported that the proton conductivity in tested electrodes starts to drop when the ionomer volume fraction becomes smaller than 13\%. A transmission line model (TLM) was developed to represent the governing equations [74]. Jang et al. [27] have presented a complex capacitance analysis for estimating the catalyst/ionomer interfacial capacitance and the ionic resistance. They concluded that the resistance is higher for MEAs with lower ionomer contents.

Legros et al. [75] employed ex-situ EIS and acoustic emission methods to estimate the water amount in the membrane. In the acoustic emission method, the sample is exposed to a produced wave. The elastic energy of the sample is determined by measuring the sample surface displacement using piezoelectric sensors attached to the sample surface. Based on the data, they reported that the acoustic emission technique is more sensitive to drying processes (compared to EIS). Huth et al. [76] estimated the proton transport of the membrane of a high temperature PEM fuel cell by feeding the pure nitrogen to the cathode and measuring the impedance. They presented the fuel cell Bode diagrams at different pressures and temperatures. They claimed that the proton transport is the limiting process when there is enough hydrogen supply at the anode. 
Navarro et al. [77] used a pore-filling electrolyte membrane fabricated based on plasmaactivated microporous polyethylene matrices to study the performance of the fuel cell. They employed EIS to measure the electrical response. They reported the Nyquist plots of single cells while feeding both electrodes with hydrogen and oxygen. In their results, the impedances of the connecting wires are dominant, especially in the hydrogen feeding plots. Tsampas et al. [78, 79] studied the proton transport mechanisms in the fuel cell membrane using EIS. They claimed there are two different mechanisms for proton conduction in the fully hydrated Nafion membranes: proton migration in the aqueous and proton transfer between adjacent sulfonate groups in narrow pores. They stated that at low hydrogen partial pressures the dominant proton transport mechanism is the second one which is the reason for the steady-state bistability performance (i.e. two different potential values corresponding to the same current density) in the fuel cells. They reported negative real part impedances with large imaginary components in the Nyquist plots for these performance conditions. Considering the fact that the real part of the impedance values in low frequencies are the negative of the slope of the polarization curves and the polarization curve has positive slope in the bistability points, it is reasonable to have negative real parts in the Nyquist plot. These negative real parts have also been reported before in metal corrosion [80, 81] and direct methanol fuel cells [82]. Furthermore they stated that the proton transport time constant can be as large as $10 \mathrm{sec}$, and hence it can be the limiting process in the fuel cell.

Lamy et al. [83] reviewed the effects of the electrochemical characteristics of the membrane on the fuel cell performance. They simulated the mass and charge transport inside the membrane and then theoretically studied the influence of the catalyst layer and GDL porosity on the impedance diagrams. As part of their study, they optimized the operating parameters which resulted in the fabrication of their new membrane electrode assembly.

Finally, it has to be reemphasized that there are other studies on the membrane which employed EIS besides other methods, like X-ray diffraction, transmission electron microscopy, scanning electron microscopy and cycle voltammetry. In these studies, the EIS results are only used to verify measurements of the other methods [84-86].

\subsubsection{Contamination}

The use of EIS in studying contamination of electrodes have been summarized by Yuan et al. [8] who have thoroughly reviewed the effects of $\mathrm{CO}, \mathrm{NH}_{3}$ and $\mathrm{H}_{2} \mathrm{~S}$ contaminants on the fuel cell. $\mathrm{Li}$ 
et al. [87] used EIS to study the effects of relative humidity, Pt loading, and toluene contaminations on the air side electrode. They reported that the contamination effects were amplified by increasing the current density, increasing the toluene concentration, decreasing the relative humidity, and decreasing the cathode-side Pt loading. Furthermore, they concluded that the contaminants have long-term effects on the fuel cell performance which cannot be fully recovered. They added the effect of the chloride contamination in their following study [88] and found out that the cell voltage experiences an initial sudden drop followed by a plateau due to chloride contamination. Martinez-Rodriguez et al. [89] also studied the effect of chlorinated impurities on the fuel cell performance. They reported that even $0.05 \mathrm{ppm}$ impurity decreases the cell performance rapidly for the voltage range above $0.2 \mathrm{~V}$ below which poisoning has no effect. Nakajima et al. [90] studied the effects of CO poisoning on the polarization curves and impedance plots. They fed the anode with a mixture of $\mathrm{CO}$ and $\mathrm{H}_{2}$ and conducted anode impedances and I-V measurements. They reported that that the output voltage depends on $\mathrm{CO}$ poisoning.

The nonlinear frequency response analyzer (NFRA) has been used to diagnose simultaneously the processes of membrane dehydration, fuel cell flooding and anode $\mathrm{CO}$ poisoning [91]. This method, which applies high amplitude sinusoidal perturbations to the system and measures the response, is highly sensitive to distinguish between phenomena having approximately the same time constants. For instance, it is claimed that $\mathrm{CO}$ poisoning and dehydration cannot be distinguished from the EIS results; whereas, they are totally distinct in NFRA. Kadyk et al. [92] studied the anodic $\mathrm{CO}$ poisoning in a symmetrical $\mathrm{H}_{2}$ feeding cell by the polarization curves, EIS and NFRA. They used a theoretical model from the literature and simulated the NFRA results. The experimental measurements and simulated results were in good agreement. They also employed the $\mathrm{H}_{2} / \mathrm{H}_{2}$ feeding system to study the spectra related to the beginning of $\mathrm{CO}$ poisoning in the anode during fuel cell operation and reported an additional arc in the Bode plot in low frequencies at $100 \mathrm{ppm}$.

Andreasen et al. [17] studied the effect of operating temperature, current density and CO and $\mathrm{CO}_{2}$ contaminants in the anode input gas on the performance of the high temperature PEM fuel cells. They reported that the contaminants affect the entire range of the frequency spectra. The results showed that the high frequency part of the Nyquist plot was affected mostly by poisoning. They repeated every measurement two times and presented the RMS values. They declared that the discrepancies between most measurement pairs were less than $2 \%$. It is necessary to 
reemphasize that there is no clear connection between the fuel cell governing equations and measurement models, and hence the physicochemical interpretations of the presented equivalent circuits need to be reinvestigated [88, 90, 93].

\subsubsection{Aging and durability}

Roy et al. [94] studied the membrane aging by different physical and electrochemical techniques including EIS. They reported that the low-frequency inductive features are related to the phenomenon of aging. They also reported that the high frequency part of the impedance is dependent of the active surface area. Nara et al. [95] studied the degradation of the cathode catalyst layer using EIS, transmission electron microscopy, field emission scanning electron microscopy, cyclic voltammetry, micro-Fourier transform infrared spectroscopy, and nitrogen adsorption-desorption measurements. They presented a new transmission line model (TLM) as the equivalent circuit for the cathode catalyst layer considering the distribution of the oxygen reduction reaction in the pores inside and outside the agglomerates (primary and secondary pores). The presented model agrees well with the measured impedances; however, this agreement can be the result of the high number of elements used in the proposed equivalent circuit which causes high degrees of freedom of the circuit.

Fouquet et al. [24] reported the state-of-health of a PEM fuel cell in a qualitative manner. They considered the state-of-health of the fuel cell based on the dehydration and flooding conditions. However, the state-of-health has to be defined in a more general manner to cover other aspects of fuel cell performance (poisoning, aging, etc.). There are also other studies on aging and degradation using EIS. However, these studies do not present a clear connection between the elements of the measurement-model-based equivalent circuits and the physical and chemical properties of the fuel cell [96-101].

\subsubsection{Dehydration and flooding}

The EIS technique has also been used to study flooding and dehydration in the fuel cell. One of the examples is the work of Fouquet et al. [24] who reported that flooding and dehydration in the PEM fuel cell cannot be distinguished from the polarization curves. They modified the Randles classic equivalent circuit by changing the double layer capacitance with a constant phase element (CPE) and found the best fit between the model and the measured impedances. They assumed the power in the definition of CPE to be constant to have a physical meaning and avoid an 
additional degree-of-freedom in data fitting algorithms. Based on the proposed equivalent circuit, they measured the membrane polarization and diffusion resistances and defined three subspaces that are related to the nominal, flooded and dry conditions. These subspaces can be used to predict the flooding and dehydration conditions from the impedance measurements.

Bautista et al. [102] presented a model to predict the impedance response and the polarization curve of the PEM fuel cell. They considered reactant and products mass transfer in the channels and water transport across the membrane. Using the simulated Nyquist plot, they studied the effect of the dew point temperature (humidity), membrane thickness, cathode gas backing porosity, and agglomerate thickness. They were able to distinguish between the flooding and dehydration effects using impedance responses.

Kurz et al. [103] used EIS to diagnose the flooding and dehydration phenomena. From the results, they concluded that the measurement of the imaginary part of the impedance at $0.5 \mathrm{~Hz}$ and real part of the impedance at $1 \mathrm{kHz}$ will be sufficient to distinguish between flooding and dehydration. They called these two points "Flooding Indication Value" and "High Frequency Resistance", respectively. Measuring these two impedances, they predicted flooding and drying nearly half an hour prior to the time required to be observed in the polarization curves. Based on the diagnosis, they presented an equation and a control parameter that can adapt the air flow rate of the tested fuel cell stack to prevent flooding and drying. The presented control algorithm can be modified for various operating conditions, temperatures, current densities and relative humidities.

Roy and Orazem [104] reported that flooding can be predicted based on the standard deviations of the measured impedances. They stated that the formation and removal of water droplets are stochastic processes, and hence, can be predicted by a technique oriented towards deviation measurements. They claimed that the cell impedance and impedance measurement deviations increase considerably during flooding. This increase is more at the real part of the impedance and low frequencies which is close to the flooding characteristic frequency. In their next study [105], they presented a graphical method (estimating the parameters graphically) to determine the constant phase element (CPE) coefficients in high frequencies. They stated that decreasing this capacitance is a sign of flooding and drying as well. They measured the impedances at different current densities, times, temperatures, back pressures, and for different flow channels and gas diffusion layers. 
Schneider et al. [106] studied the impedance responses of the dehydrated and fully humidified Nafion membrane using EIS and current step measurements. They reported that the low frequency inductive loop in the Nyquist plot is due to the change in conductivity of the dehydrated membrane and slow uptake and release of water in the low-humidity membrane. They reported that the time constant of the membrane hydration by the by-product water is around $25 \mathrm{sec}$.

Rodat et al. [107] studied flooding and drying of a fuel cell stack and individual cells using EIS in various operating conditions. However, there are some concerns about their frequency range and equivalent circuit. The considered frequency range $(3 \mathrm{~Hz}-5 \mathrm{kHz})$ cannot cover all the phenomena in the fuel cell. Kadyk et al. [91] distinguished flooding, membrane dehydration and $\mathrm{CO}$ poisoning simultaneously using the nonlinear frequency response analysis (NFRA) and concluded that dehydration and flooding could be diagnosed clearly from the linear response analysis of the EIS measurements.

\subsubsection{Other phenomena diagnosed by EIS}

There are other studies in which other aspects and elements of fuel cells have been monitored using EIS. These examples include the structure of the flow channels, mass transport in the cathode side, clamping effect and the stack performance. For instance, Kwon et al. [108] studied three different serpentine channels using EIS. The channels were modeled by computational fluid dynamics methods and then the resistances were compared by impedance measurements. They reported that their EIS-based conclusions are in good agreement with their previous experimental and modeling results.

Mainka et al. [109] analyzed mass transfer in the cathode of the PEM fuel cell. They claimed that the limiting layer for mass transport is the active layer, and the mass transport resistance of the GDL in a normal condition is negligible. They proposed that the Warburg element has to be in series with the membrane resistance and the only parallel elements have to be the double layer capacitance and charge transfer resistance. In their next papers [110, 111], they discussed about the accuracy of the constant oxygen concentration assumption at the gas channel/GDL interface. They considered the gas consumption along the channel which enabled them to capture the local variations of the impedance spectra and diffusion kinetics. 
Asghari et al. [112] studied the effect of the clamping torque (in terms of magnitude and nonuniformity) and the operating temperature on the fuel cell performance and break-in procedure (referred to as a condition providing enough hydration in the membrane and electrodes before the peak performance point) using EIS. They claimed that non-uniformity of the clamping torque has inductive characteristics at high frequencies and can increase the ohmic resistances observed in the Nyquist plots. They stated that the inductive effects may be due to the non-uniform current distribution especially within the GDL. They presented the optimum clamping torque based on the variations observed in different resistances. Furthermore, they claimed that increasing the operating temperature decreases the charge transfer resistance, and also the EIS method can be employed to monitor the break-in process.

The impedance measurement has also been conducted on the fuel cell stacks or one special single cell in the stack [e.g., 15, 16, 36, 103, 113-115]. There are remarkable studies on the fuel cell stack accessories or the effect of the cell position $[15,115]$. For instance, the work of Dale et al. [15] shows that the ohmic resistance depends on the cell location and the lowest ohmic resistance observed for the closest cell to the air inlet. They have also measured the capacitor or inductor effects at high frequencies and showed that the inductor effects occur for cells close to the hydrogen inlet. Finally, various accelerated conditioning methods for PEM fuel cells using different experimental techniques including EIS were compared by Yuan et al. [115].

\subsection{Measurement, semi-process and process modeling}

As mentioned before and reviewed above, despite the similarity that exists between the two ways of determining the equivalent circuit, the measurement and process models can produce quite different results in a sense that the circuit obtained from the governing equations in a process model has a physical meaning (presenting the phenomena that occur in the cell) and can be applied to a wide range of operating conditions; whereas the assumed equivalent circuit in a measurement model does not possess these properties. Despite this difference, measurement and process models are often not distinguished from each other in the literature, which results in reaching to dubious conclusions [e.g., 49-58, 66-69, 84-86, 88, 90, 93, 96-101]. In this section, these two modeling approaches are thoroughly discussed. The procedure for obtaining the equivalent circuit from the analytical solution and the importance of such a procedure, which distinguishes the process models from other analytical models, are also outlined. In addition, the 
new semi-process model category, presenting the equivalent circuit in a different way than the process models, is introduced and discussed.

\subsubsection{Measurement modeling approach}

Except for a few studies published in the past [e.g. 74, 80, 102, 109, 110, 117-124], all the equivalent-circuit-based studies conducted on PEM fuel cells can be categorized as the measurement models. In a measurement model, basically, the impedance of the fuel cell is first measured and then an equivalent circuit with the impedance characteristics similar to those of the measurements is assumed. There is a wide range of different impedance modeling packages (ECLab, ZView, etc.) which present different pre-built equivalent circuits that can be used to model the measured impedances. All of these models have to be classified as measurement models.

The concept of the measurement model was first presented by Agarwal et al. [9, 19] as a better way of quantifying the measured impedances compared to other regression methods (such as polynomial fitting) [9]. The significance of the measurement model (compared to other regression methods) is that the assumed equivalent circuit satisfies the Kramer-Kronig relations $[9,125]$ and simplifies the detection of stochastic errors [19], guarantying the accuracy of the measured impedances. Thus, the measurement models can be used as a tool for estimating the accuracy of the measured impedances. In fact, if the impedance of the assumed equivalent circuit fits well to the measured impedance, it can be concluded that the measured impedance satisfies the Kramer-Kronig relations (see the detailed discussion presented by Agarwal et al. [9, 19]). Despite this advantage of the measurement models over other regression methods, it is still not wise to perform any physical or chemical interpretations from the changes in the values of the elements of the assumed circuit $[1,9,19,120,126]$. In fact, there is no physical or theoretical relation between the elements of these hypothetical equivalent circuits and the physical and chemical properties of the PEM fuel cell. For instance, the values of the elements of the equivalent circuit assumed for the measurement model of a cell can change due to the change in the measured impedance of the cell occurring by replacing the membrane. In this case, no physical or theoretical interpretations should be concluded based on the change in the value of the elements of the equivalent circuit since it is not certain whether this change is due to the change in the membrane thickness, conductivity, or an unknown effect that might not be even related to the membrane (e.g., oxygen transfer to the catalyst layer). Thus, most physical discussions conducted this way could be considered as misinterpretations and need to be re- 
evaluated [1]. Although the measurement-model approach was not essentially presented for concluding these kinds of physical discussions, majority of different models were presented in literature as a result of this misinterpretation, which all can be categorized as measurement models. Despite this conflict, the measurement-model approach still presents a strong methodology for quantifying and studying the measured impedances. It can also be used as a stepping stone to derive the equivalent circuit from the process models [9].

\subsubsection{Process modeling approach}

In the process-model approach, the impedance of the cell is analytically determined by perturbing the governing equations which is finally converted to an equivalent circuit. The equivalent circuit has the same impedance characteristics as the impedance obtained analytically. The most famous example is the Randles process model determined based on a set of governing equations (i.e., the linear diffusion and Butler-Volmer equations), modeling the reaction of a metal ion in an aqueous solution [21]. Since the Randles equivalent circuit was not derived from measurement models, there is a clear mathematical relation between the physicochemical properties of the system and the elements of the equivalent circuit. Thus, the resulting equivalent circuit can be used for all similar systems and for all various operating conditions.

\subsubsection{Procedure for obtaining the analytical solutions}

The final goal of the analytical calculations in the process model is to find the analytical solution for the impedance of the cell $[11,21,127,128]$. The approach is similar to the procedure used in the measurement of the impedance of the cell. A small harmonic perturbation is applied to the system resulting in perturbing the parameters in the governing equations which are related to the cell characteristics. The perturbation parts are assumed to be small enabling the linearization of the equations in which the second order terms are neglected. The impedance can then be determined as the ratio of the perturbed part of the potential to the perturbed part of the current. It has to be mentioned that in this impedance relation there is no perturbed parameters. The time derivatives of the perturbed parts of the parameters in the equations are also replaced by the

perturbed part itself multiplied by $(i \omega)$, where $\omega$ is angular frequency and $i=\sqrt{-1}$. These terms result in the capacitive and inductive elements in the final equivalent circuits which will be discussed later in Section 1.2.2.3. 


\subsubsection{Importance of the equivalent circuit}

After obtaining the analytical relation for the impedance, the equivalent circuit can be determined. The equivalent circuit is not derived to solve or verify the analytical model. The sole purpose of deriving the equivalent circuit is to represent the equations in an alternative way. It can be considered as a transformation to another domain which makes the interpretations easier (conceptually, similar to Laplace or Fourier transforms). In addition, the derivation of the equivalent circuit has several advantages:

i) It is considerably easier to deal with an equivalent circuit rather than the final analytical solution. While the impedance equation does not highlight the physicochemical effects, the equivalent circuit can be interpreted physically and its elements can be labeled or even recognized from prior theoretical studies.

ii) The equivalent circuit is an excellent tool to combine the parameters included in the final analytical equation and to present them as components that are physically or chemically meaningful. As an example, if all the parameters related to a specific part of the cell (e.g., membrane) are just appeared in one element of the equivalent circuit, it can be concluded that it is only that specific element which is related to the membrane. Also, if all the physical and chemical parameters related to a specific phenomenon is appeared in an element or a block of elements, that part could be separated to study the phenomenon independently.

iii) The equivalent circuit elements and combinations are familiar to researchers in electrochemistry. For example, it is known that an $\mathrm{R}-\mathrm{C}$ parallel connection causes a semicircle in the Nyquist plot. So, if the process model is presented by an R-C parallel equivalent circuit, it can be concluded at first sight that the impedance of the analytical solution should be a semi-circle in the Nyquist plot, which facilitates the comparison between the analytical solution and the measured impedances. Clearly, no other combination of elements can make a semi-circle. Thus, if the process model is shown by another equivalent circuit (e.g., a series combination of elements), it is undoubtedly wrong. The paper published by Springer et al. [117] shows an example of this issue. In essence, if the impedance model is not solved analytically, and hence an equivalent circuit is not derived, it is not clear what impedance patterns in the Nyquist plots can be produced by the model and how the fuel cell parameters can be related to different parts of the predicted Nyquist plots. 
iv) The applicability and physical interpretations of a process model are directly linked to the presentation of the equivalent circuit. This can be clarified in the following example: assume that the impedance of an electrochemical reaction (modeled even by an extremely accurate process model that is not presented by an equivalent circuit) consists of two separate semi-circles (in the Nyquist plot) in the high and low frequency ranges. If one of the operating conditions of the measurement changes resulting in inflation or shrinkage of the high-frequency semi-circle, it would be extremely hard, if not impossible, to identify the parameter which causes this inflation or shrinkage. However, if the equivalent circuit is known, it can be observed that the resistance in the parallelconnected R-C elements related to this frequency range has been changed so that the parameter causing this change can readily be determined.

\subsubsection{Procedure for obtaining the equivalent circuit}

The procedure followed for converting the analytically determined impedance to an equivalent circuit is not a trivial task. To our best knowledge, there are no predefined mathematical steps to do this conversion. Although it is mostly based on innovative approaches taken in each specific solution, there are general points (see below) which can be used as guidelines:

- If the equation can be written as the summation of two blocks, those two blocks are connected in series.

- If a block does not include any $(i \omega)$ term, it can be considered as a resistance element in series with the remaining part [128].

- If a block $D$ in the equation is defined in a fashion that the equation can be reorganized as $\frac{D}{1+D c i \omega}$, the block $D$ is in parallel with the capacitance $c$.

- If the equation consists of $(i \omega)^{2}$ terms, it means that two capacitances, inductances or combinations of them are involved [128].

- Finally, it has to be mentioned that in some complex equations, more than one equivalent circuit could be possible. While all of the solutions are mathematically correct, only the solution for which all the resistive, capacitive and inductive elements have positive values, is physically meaningful. Moreover, the equivalent circuit which has elements satisfying previous theoretical studies (e.g. double layer 
capacitance) should be preferred. In general, the equivalent circuit should be selected wisely in each specific system and modeling purpose.

\subsubsection{Semi-process modeling approach}

There are a few published papers in the impedance modeling of PEM fuel cells which have to be considered in a separate category than the process modeling approaches [e.g. 74, 80, 102, 109, 110, 118-121, 124]. These models are categorized as semi-process models here. In this modeling category, the governing equations are first studied and solved analytically. Then, an equivalent circuit is "assumed" and the elements of this equivalent circuit are determined from the analytical solution. In other words, while the analytical calculations are similar to the processmodeling approach, the equivalent circuit is selected similar to the measurement-modeling approach. This equivalent circuit normally consists of two R-C parallel connections for electrodes which are in series with a resistance considered for the membrane. While the verification of the presented models based on the measured impedances is normally restricted to a limited number of cases, the assumed equivalent circuit imposes its own impedance characteristics on the model. For instance, the above mentioned equivalent circuit can only present two connected semi-circles in the Nyquist plot which are shifted on the real axis. While these two semi-circles can be convoluted to one semi-circle, the various characteristics of the measured impedances (such as three separate semi-circles [20,34, 46, 114] or inductive effects $[91,114]$ ) cannot be predicted or explained fundamentally. For PEM fuel cells, there is no complete process model similar to those presented for other electrochemical systems (e.g. [11, 21, 127]). Springer et al. [117] presented the most cited model for impedance characteristics of a PEM fuel cell. The set of equations were solved numerically in their solution process, and hence, no equivalent circuit was presented. As a result, the impedance patterns predictable by their model and also the relation between the fuel cell parameters and different parts of the resultant Nyquist plots are not clear. Similar to other studies [122, 123], Springer et al. compared their model predictions with the measured impedances for a wide range of operating conditions; however, it is still not accurate. All the other attempts towards the impedance modeling of PEM fuel cells $[74,80,102,109,110,118-121,124]$ have to be categorized as semi-process models as the equivalent circuits are not determined from the analytically calculated impedance. While the assumed equivalent circuits are normally based on the process models presented for similar electrochemical systems, the complex structure of the PEM fuel cell cannot precisely be captured using these models. Thus, some characteristics of the measured impedances cannot be covered. 


\subsection{Motivations and objectives}

As mentioned in the previous section, while different research groups analytically studied the impedance characteristics of PEM fuel cells, no complete process model has been published in literature. The process model and its resultant equivalent circuit can clarify the physical parameters governing each part of the measured impedances, and as a result, it can clearly explain what kind of information can be extracted from the impedances and what aspects of the working conditions are not covered by the measured impedances.

In this research, the first complete process model for PEM fuel cells is presented. The impedance of the cell is separated based on different losses of the cell. Each loss is studied separately and the corresponding process model and equivalent circuit is determined. The models are then combined and the complete process model of the PEM fuel cell is obtained and verified against the impedances measured in different operating conditions. Using the verified model, the measured Nyquist plots of the PEM fuel cells reported in literature are categorized. As a result, the dominant physical and chemical parameters controlling various arcs of the Nyquist plot are determined. The thesis is organized as follows:

The process model resulted from the ohmic loss of the PEM fuel cell is presented in Chapter 2. The analytical analysis and the corresponding equivalent circuit are discussed. The model predictions are then compared against the measured impedances reported in literature.

Chapter 3 covers the process modeling of the activation and mass transport losses. The required analytical analyses for the anode and cathode electrodes are presented separately and the equivalent circuits are obtained. Then, the predictions of the model are compared against the measured impedances reported in literature.

The obtained process models and equivalent circuits are combined in Chapter 4 to determine the complete equivalent circuit of the PEM fuel cell. The model is then verified using the cell impedances measured in different operating conditions using our fuel cell hardware and test station. Next, the Nyquist plot of the cell is divided into the separate sections and each section is thoroughly discussed and the governing parameters are presented.

The sensitivity of the impedance characteristics of the cell to the variations in the operating conditions are studied in Chapter 5. The effects of the current density on measured impedances are canceled using the model. It makes the sensitivity analysis possible which results in 
determining the required precision in the impedance measuring systems. This analysis along with the definition and calculation of the "indifference interval" are presented in this chapter.

To show the range of the applicability of the model, the anode and cathode starvation effects are discussed in Chapter 6. It is discussed that the model predicts limitations of the EIS on detecting the anode starvation which was not reported before in literature. However, measuring the impedance of the cell in the starved case completely confirms the predictions. These measured impedances and the model predictions are presented and discussed in Chapter 6.

Finally, Chapter 7 summarises the concluding remarks of this research and proposes the recommendations for future studies on this subject using the potentials of the model presented. 


\section{Chapter 2. Process model for ohmic loss ${ }^{1}$}

The impedance characteristics of the ohmic overpotential of the proton exchange membrane (PEM) fuel cells are studied analytically using the process modeling approach. The water transport in the membrane, the cathode catalyst layer, and gas diffusion layer are analyzed. The analytical relation of the impedance of the ohmic loss is determined and is converted to an equivalent circuit. The model predictions are then compared against the measured impedances reported in literature. It also shows that the membrane dehydration can be detected from the high frequency resistance of the cell.

\subsection{Theory}

The output voltage of a PEM fuel cell $(V)$ can be presented as [2]

$V=E_{\text {theor. }}-\eta_{\text {act. }}-\eta_{\text {ohm. }}-\eta_{\text {trans. }}$

In this equation, $V$ and $E_{\text {theor. }}$ are the real voltage output and the theoretically predicted voltage, respectively. $\eta_{a c t}, \eta_{\text {ohm }}$ and $\eta_{\text {trans }}$ present the activation losses due to reaction kinetics, the ohmic losses due to ionic and electronic conduction, and the concentration losses due to mass transport, respectively. To calculate the impedance, each of the losses has to be analytically determined as a function of the cell current. The ohmic loss is studied in this chapter, while the activation and transport losses are modeled in Chapter 3.

The ohmic overpotential of a PEM fuel cell can be estimated as

$\eta_{o}=j A\left(R_{e}+R_{i}\right)$

\footnotetext{
${ }^{1}$ Parts of Chapter 2 have been published in a journal article. Reprinted with permission from [128].
} 
where $j, A, R_{e}$ and $R_{i}$ denote the current density, the active area, the electronic and the ionic resistances, respectively. The electronic resistance $\left(R_{e}\right)$ is normally several orders of magnitude smaller than the ionic resistance $\left(R_{i}\right)$, and hence, it can be safely neglected $[2,128]$. Considering the triple phase boundary concept $[2,129]$, the ionic resistance is essentially the result of the proton transfer in the membrane and could be formulated based on the membrane conductivity $\left(\sigma_{m}\right)$ which is itself a linear function of the water content of the membrane $\left(\lambda_{m}\right)[2,130]$

$\sigma_{m}=a \lambda_{m}-b$

where

$$
\begin{aligned}
& a=0.5193 \exp \left[1268\left(\frac{1}{303}-\frac{1}{T}\right)\right] \\
& b=0.326 \exp \left[1268\left(\frac{1}{303}-\frac{1}{T}\right)\right]
\end{aligned}
$$

The membrane water content $\left(\lambda_{m}\right)$ is defined as the ratio of the number of molecules of water in the membrane to the number of charged sites. It can be formulated based on the water concentration as [2]

$$
\lambda_{m}=\frac{M_{m}}{\rho_{m}} c_{m}
$$

where $M_{m}, \rho_{m}$ and $c_{m}$ are the membrane equivalent weight, the membrane dry density and the water concentration in the membrane, respectively. The ohmic overpotential can therefore be written as

$$
\eta_{o}=\frac{l_{m}}{\sigma_{m}} j=\frac{l_{m}}{a \lambda_{m}-b} j
$$

To determine the impedance of the ohmic loss in different frequencies, the parameters are perturbed using a sinusoidal perturbation. The changes in the values of the parameters due to the perturbation are represented with the symbol $\sim\left(\right.$ e.g. $\left.\tilde{\lambda}_{m}\right)$, while the parameter values prior to applying the perturbation is shown by the symbol ${ }^{-}$(e.g. $\bar{\lambda}_{m}$ ). Clearly, when the perturbation is applied 
$\lambda_{m}=\bar{\lambda}_{m}+\tilde{\lambda}_{m}$

Moreover, as the perturbations are assumed to be harmonic, the time derivate of the perturbed parts (e.g. $\tilde{\lambda}_{m}$ ) can be written as

$\frac{\partial \tilde{\lambda}}{\partial t}=i \omega \tilde{\lambda}$

where $\omega$ is angular frequency and $i=\sqrt{-1}$ [128]. Considering the above definitions, Equation (2.7) can be rearranged as

$\bar{\eta}_{o}+\tilde{\eta}_{o}=\frac{l_{m}}{a\left(\bar{\lambda}_{m}+\tilde{\lambda}_{m}\right)-b}(\bar{j}+\tilde{j})$

Using the Taylor series expansion and small perturbation assumption and so neglecting the second and higher orders of the perturbed parameters, one can find

$\bar{\eta}_{o}+\tilde{\eta}_{o}=\frac{l_{m}}{a \bar{\lambda}_{m}-b} \bar{j}+\frac{l_{m}}{a \bar{\lambda}_{m}-b} \tilde{j}-\frac{l_{m}}{a \bar{\lambda}_{m}-b} \frac{a \bar{j}}{a \bar{\lambda}_{m}-b} \tilde{\lambda}_{m}$

Considering the following relations:

$\bar{\eta}_{o}=\frac{l_{m}}{\bar{\sigma}_{m}} \bar{j}$

$\bar{\sigma}_{m}=a \bar{\lambda}_{m}-b$

the perturbed part of the ohmic overpotential $\left(\tilde{\eta}_{o}\right)$, can be determined as

$\tilde{\eta}_{o}=\frac{l_{m}}{\bar{\sigma}_{m}} \tilde{j}-\frac{l_{m} a \bar{j}}{\bar{\sigma}_{m}^{2}} \tilde{\lambda}_{m}$

As a result, the impedance of the ohmic loss $\left(Z_{o}\right)$ can be calculated as

$Z_{o}=\frac{\tilde{\eta}_{o}}{\tilde{j}}=\frac{l_{m}}{\bar{\sigma}_{m}}-\frac{l_{m} a \bar{j}}{\bar{\sigma}_{m}^{2}} \frac{\tilde{\lambda}_{m}}{\tilde{j}}$

As Equation (2.15) demonstrates, the impedance of the ohmic overpotential consists of an ordinary resistance with the value of $l_{m} / \bar{\sigma}_{m}$ and another term which is a function of $\tilde{\lambda}_{m} / \tilde{j}_{j}$. This 
term is calculated in the following sections and the final impedance equation and equivalent circuit are determined.

\subsubsection{Water transport in the membrane}

Assuming a one-dimensional water flux in the membrane, the conservation of mass results in

$$
J_{m}=l_{m} \frac{\partial c_{m}}{\partial t}
$$

where $J_{m}$ is the water flux in the membrane. Considering the water flux caused by the proton transfer in the membrane and back diffusion $[2,128]$ and using Equation (2.6), one can show

$$
\frac{l_{m} \rho_{m}}{M_{m}} \frac{\partial \lambda_{m}}{\partial t}=-\alpha j \lambda_{m}+\beta \frac{\lambda_{c l}-\lambda_{m}}{l_{m} / 2}
$$

Here, $\alpha$ and $\beta$ are $[2,128]$

$$
\begin{aligned}
& \alpha=\frac{2.5}{22 F} \\
& \beta=\frac{\rho_{m}}{M_{m}} D_{m}
\end{aligned}
$$

where $D_{m}$ represents the membrane diffusion coefficient, and $F$ is Faraday's constant (96485 $\mathrm{C} \cdot \mathrm{mol}^{-1}$ ) [2]. In Equation (2.17), $\lambda_{c l}$ is called the water content of the cathode catalyst layer and defined as

$$
\lambda_{c l}=c_{c l} \frac{M_{m}}{\rho_{m}}
$$

It has to be mentioned that while $\lambda_{m}$ has a physical meaning and represents the ratio of the number of the water molecules to the number of $\mathrm{SO}_{3}^{-} \mathrm{H}^{+}$sites [2]; there is no physical interpretation for $\lambda_{c l}$. In fact, it was defined similar to the membrane water content to simplify the deriving process. As water production in the PEM fuel cell occurs in the cathode, the water transport in the cathode catalyst and gas diffusion layers are considered and modeled here. It is clear that the model can be improved by adding the anode water transport modeling; however, the agreement between the predicted impedances of this model with the measured impedances - 
which is discussed in Section 2.2 - shows that the anode water transport does not have dominant effects.

Equations (2.16) and (2.17) are also based on the assumption that the water concentration in the membrane is spatially constant. In fact, Equation (2.17) has to be corrected as

$\frac{\rho_{m}}{M_{m}} \frac{\partial \lambda_{m}}{\partial t}=-\alpha j \frac{\partial \lambda_{m}}{\partial x}+\beta \frac{\partial^{2} \lambda_{m}}{\partial x^{2}}$

In the steady state condition (prior to applying the perturbation), the left hand side of this equation vanishes, and as a result, the water content of the membrane can be calculated as

$\bar{\lambda}_{m}(x)=\left(\bar{\lambda}_{c l}+\frac{\bar{J}_{m}}{\alpha \bar{j}}\right) \exp (x \alpha \bar{j} / \beta)-\frac{\bar{J}_{m}}{\alpha \bar{j}}$

where $\bar{J}_{m}$ is the net water flux from the membrane to the cathode catalyst layer. Considering this distribution, the average value of the water content in the membrane can be determined as

$$
\bar{\lambda}_{m, a v g}=\frac{\bar{\lambda}_{c l}+\bar{J}_{m} /(\alpha \bar{j})}{l_{m} \alpha \bar{j} / \beta}\left(\exp \left(l_{m} \alpha \bar{j} / \beta\right)-1\right)-\frac{\bar{J}_{m}}{\alpha \bar{j}}
$$

To estimate the significance of the spatial distribution of the membrane water content, the point $(x=\zeta)$ in the membrane at which the membrane water content becomes equal to the average water content presented in Equation (2.23) is considered. In other words, $\zeta$ is determined such that

$$
\bar{\lambda}_{m}(x=\zeta)=\bar{\lambda}_{m, a v g}
$$

It can be shown that

$$
\frac{\zeta}{l_{m}}=\frac{\ln \left(\exp \left(l_{m} \alpha \bar{j} / \beta\right)-1\right)-\ln \left(l_{m} \alpha \bar{j} / \beta\right)}{l_{m} \alpha \bar{j} / \beta}
$$

As the water content distribution is exponential (Equation (2.22)), the closer the above ratio $\left(\zeta / l_{m}\right)$ to the extreme values of 0 and 1 , the more significant the spatial water distribution. In other words, the $\left|\frac{\zeta}{l_{m}}-0.5\right|$ value can be used to estimate the significance of the spatial distribution. It can be shown that this value is considerably small and the assumption of the constant water content in the membrane is valid unless when the current density is high and 
simultaneously the water diffusion coefficient of the membrane is extremely low. In normal cases, the error caused by neglecting the spatial distribution of the water content of the membrane is less than $2 \%$.

Considering Equations (2.8) and (2.9), the perturbed part of the water content of the membrane $\left(\tilde{\lambda}_{m}\right)$, can be determined from Equation (2.17) as

$\frac{l_{m} \rho_{m}}{M_{m}}\left(i \omega \tilde{\lambda}_{m}\right)=-\alpha \bar{j} \tilde{\lambda}_{m}-\alpha \bar{\lambda}_{m} \tilde{j}+\frac{2 \beta}{l_{m}} \tilde{\lambda}_{c l}-\frac{2 \beta}{l_{m}} \tilde{\lambda}_{m}$

which can be rearranged as

$\left[\frac{l_{m} \rho_{m}}{M_{m}} i \omega+\left(\alpha \bar{j}+\frac{2 \beta}{l_{m}}\right)\right] \tilde{\lambda}_{m}=-\alpha \bar{\lambda}_{m} \tilde{j}+\frac{2 \beta}{l_{m}} \tilde{\lambda}_{c l}$

To find $\tilde{\lambda}_{m}$ from this equation, $\tilde{\lambda}_{c l}$ has to be determined first. This parameter is estimated in the following section.

\subsubsection{Water transport in the cathode catalyst and gas diffusion layers}

The water concentration in the cathode catalyst layer can be estimated as

$\frac{\partial c_{c l}}{\partial t}=\frac{j}{2 F l_{c l}}-\frac{F_{\mathrm{GDL}}}{l_{c l}}+\frac{J_{m}}{l_{c l}}$

where $F_{\mathrm{GDL}}$ and $l_{c l}$ denote the water flux in the cathode gas diffusion layer and the thickness of the cathode catalyst layer, respectively. The first term in the right hand side of the Equation (2.28) comes from water production due to the chemical reaction. The second and third terms represent the water flux from the catalyst layer to the gas diffusion layer and the membrane, respectively. Using Equations (2.9), (2.16) and (2.20), the perturbed part of the water content of the cathode catalyst layer $\left(\tilde{\lambda}_{c l}\right)$ can be determined as

$\frac{\rho_{m}}{M_{m}} i \omega \tilde{\lambda}_{c l}=\frac{1}{2 F l_{c l}} \tilde{j}-\frac{1}{l_{c l}} \tilde{F}_{\mathrm{GDL}}+\frac{l_{m} \rho_{m}}{l_{c l} M_{m}} i \omega \tilde{\lambda}_{m}$

The water flux in the cathode gas diffusion layer can be modeled using the effective diffusion coefficient concept which includes both diffusion and capillary motion phenomena [128, 131133] as 


$$
F_{\mathrm{GDL}}=D_{\mathrm{GDL}}^{w} \frac{c_{c l}-c_{c h}}{l_{\mathrm{GDL}}}
$$

In this equation, $D_{\mathrm{GDL}}^{w}, l_{\mathrm{GDL}}$ and $c_{c h}$ stand for the effective water diffusion coefficient, thickness of the cathode gas diffusion layer, and the water concentration in the cathode flow channel, respectively. The efficiency of the water removal of the channel can be defined as

$\eta_{c h}=\frac{c_{c l}-c_{c h}}{c_{c l}}$

This efficiency is considered to be equal to one unless the channel is clogged. Using this definition and the definition presented in Equation (2.20), the water flux of the gas diffusion layer in the cathode side can be estimated as

$$
F_{\mathrm{GDL}}=\frac{D_{\mathrm{GDL}}^{w} \eta_{c h} \rho_{m}}{M_{m} l_{\mathrm{GDL}}} \lambda_{c l}
$$

As a result, the perturbed part can be written as

$$
\tilde{F}_{\mathrm{GDL}}=\frac{D_{\mathrm{GDL}}^{w} \eta_{c h} \rho_{m}}{M_{m} l_{\mathrm{GDL}}} \tilde{\lambda}_{c l}
$$

Substituting Equation (2.33) into the Equation (2.29) results in

$$
\left[i \omega+\frac{D_{\mathrm{GDL}}^{w} \eta_{c h}}{\left(l_{c l}+f l_{\mathrm{GDL}}\right) l_{\mathrm{GDL}}}\right] \tilde{\lambda}_{c l}=\frac{M_{m}}{2 F\left(l_{c l}+f l_{\mathrm{GDL}}\right) \rho_{m}} \tilde{j}+\frac{l_{m}}{l_{c l}+f l_{\mathrm{GDL}}} i \omega \tilde{\lambda}_{m}
$$

Before inserting the above equation in the membrane water content equation (Equation (2.27)) and calculating the ohmic loss impedance, there are two points that have to be clarified. First, the catalyst layer thickness $\left(l_{c l}\right)$ is replaced here by the $\left(l_{c l}+f l_{\mathrm{GDL}}\right)$ term. The catalyst layer thickness $\left(l_{c l}\right)$ is assumed to be the thickness of the volume which has the water concentration of $c_{c l}$. However, when the water amount in the catalyst layer increases considerably (which is the case in high current densities), the concentration of water in the parts of the gas diffusion layer against the catalyst layer could be the same as the water concentration in the catalyst layer. As a result, this thickness needs to be modified and increased slightly in high current densities. The flooding parameter $(f)$ is defined here as the part of the gas diffusion layer in which the water concentration is equal to the catalyst layer water concentration. Similar pattern has to be considered for the effective diffusion coefficient of the water transport in the gas diffusion layer 
$\left(D_{\mathrm{GDL}}^{w}\right)$. The diffusion equation (Equation (2.30)) assumes that the cross-sectional area of the transport phenomenon is remained constant regardless of the concentration of the fluid diffused. Thus, the fluid flux increases linearly with the increase in the gradient of the fluid concentration. However, as the gradient of the water concentration increases in a porous medium, the number of the active transport paths for water in the porous structure increases. In other words, the "effective" cross-sectional area changes as the gradient of the water concentration changes. As a result, the effective diffusion coefficient $\left(D_{\mathrm{GDL}}^{w}\right.$ ) has also to be modified for different ranges of the current density corresponding to the water concentration in the catalyst layer. Thus, in this model, $D_{\mathrm{GDL}}^{w}$ is changed when the current density is changed considerably.

Inserting Equation (2.34) into the Equation (2.27), the perturbed part of the membrane water content $\left(\tilde{\lambda}_{m}\right)$ can be determined as

$$
\left[\frac{l_{m} \rho_{m}}{M_{m}} i \omega+\left(\alpha \bar{j}+\frac{2 \beta}{l_{m}}\right)-\frac{2 \beta}{l_{m}} \frac{\frac{l_{m}}{l_{c l}+f l_{\mathrm{GDL}}} i \omega+\frac{D_{\mathrm{GDL}}^{w} \eta_{c h}}{\left(l_{c l}+f l_{\mathrm{GDL}}\right) l_{\mathrm{GDL}}}}{i \omega} \tilde{\lambda}_{m}=\left[-\alpha \bar{\lambda}_{m}+\frac{2 \beta}{l_{m}} \frac{\frac{M_{m}}{2 F\left(l_{c l}+f l_{\mathrm{GDL}}\right) \rho_{m}}}{i \omega+\frac{D_{\mathrm{GDL}}^{w} \eta_{c h}}{\left(l_{c l}+f l_{\mathrm{GDL}}\right) l_{\mathrm{GDL}}}}\right] \tilde{j}\right.
$$

Combining Equations (2.35) and (2.15), the impedance of the ohmic loss can be calculated as

$$
Z_{o}=\frac{l_{m}}{\bar{\sigma}_{m}}-\frac{l_{m} a \bar{j}}{\bar{\sigma}_{m}^{2}} \frac{(M+U W)+U i \omega}{X(i \omega)^{2}+(X W+Y-N) i \omega+Y W}
$$

where

$$
\begin{aligned}
& U=-\alpha \bar{\lambda}_{m} \\
& M=\frac{\beta M_{m}}{l_{m} F f l_{c l} \rho_{m}} \\
& W=\frac{D_{\mathrm{GDL}}^{w} \eta_{c h}}{f l_{c l} l_{\mathrm{GDL}}} \\
& X=\frac{l_{m} \rho_{m}}{M_{m}} \\
& Y=\alpha \bar{j}+\frac{2 \beta}{l_{m}}
\end{aligned}
$$




$$
N=\frac{2 \beta}{f l_{c l}}
$$

To find the electrical circuit equivalent to the Equation (2.36), the circuit equivalent to the second part in the right hand side of the equation has to be determined. As the first part is not a function of frequency $(\omega)$, it is equivalent to an ordinary resistance which is in series connection to the block of elements equivalent to the second part (see Chapter 1).

To determine the electrical circuit equivalent to the second part in the right hand side of the Equation (2.36), several points have to be considered. First, this part can be rearranged and written as $\frac{1+O_{1} i \omega}{O_{2}(i \omega)^{2}+O_{3} i \omega+O_{4}}$, which clearly shows that the second part has four degrees of freedom. In other words, it is equivalent to an electrical circuit which has four elements. These four elements could be the combination of resistances, capacitances and inductances and/or electrochemistry-specific elements such as Warburg and constant phase elements (CPE) which are not considered here for simplicity. It is clear that the frequency-dependent terms (i.e. $O_{1}, O_{2}$ and $\mathrm{O}_{3}$ terms) are resulted from the frequency-dependent elements (i.e., capacitors and inductors). Since the highest degree polynomial between the numerator and denominator is two $\left(\mathrm{O}_{2}(\mathrm{i} \omega)^{2}\right)$, the equivalent circuit has to include two non-mergeable capacitive or inductive elements. The non-mergeable elements are defined here as the elements which cannot be replaced by one similar element with a different value. As an example, two parallel capacitors cannot be considered as non-mergeable elements as they can be replaced by an equivalent capacitor. Finally, it has to be noted that the numerator does not have the $(i \omega)^{2}$ term which must also to be considered in determining the equivalent circuit.

Considering the discussions presented above, it can be shown that the block of elements (including $R_{1}, R_{2}, C$ and $L$ elements) shown in Figure 2.1 has the similar impedance characteristics as that in Equation (2.36). In essence, the impedance of this circuit can be determined as

$$
Z_{\text {circuit }}=R_{0}+\frac{R_{1} R_{2}+R_{1} L(i \omega)}{\left(R_{1}+R_{2}\right)+\left(R_{1} R_{2} C+L\right)(i \omega)+R_{1} L C(i \omega)^{2}}
$$




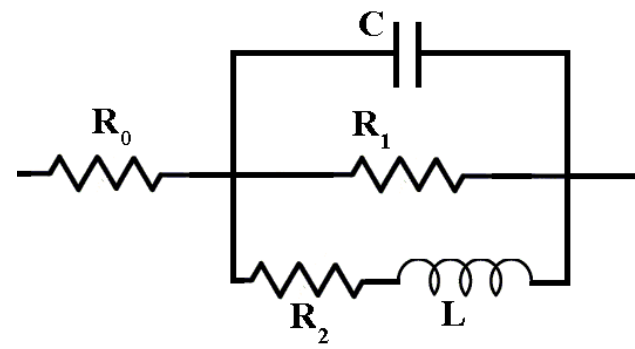

Figure 2.1 The equivalent circuit of the ohmic loss

As it can be seen in Equation (2.43), the second part of the impedance relation has the same characteristics observed in Equation (2.36). By equating the Equations (2.36) and (2.43), a set of non-linear equations is obtained [128] which results in

$$
C=\frac{X}{U}
$$

$R_{1}=\frac{U^{2}}{U Y-(U N+X M)}$

$R_{2}=\frac{U^{2}(M+U W)}{-U M Y+(M+U W)(U N+X M)}$

$$
L=\frac{U^{3}}{-U M Y+(M+U W)(U N+X M)}
$$

Finally, using the relations presented in Equations (2.37-2.42), the values of the presented equivalent circuit can be determined as a function of the fuel cell parameters.

$$
R_{0}=\frac{l_{m}}{\bar{\sigma}_{m}}
$$

$$
C=\frac{\bar{\sigma}_{m}^{2}}{l_{m} a \bar{j}} \frac{l_{m} \rho_{m}}{\alpha \bar{\lambda}_{m} M_{m}}
$$

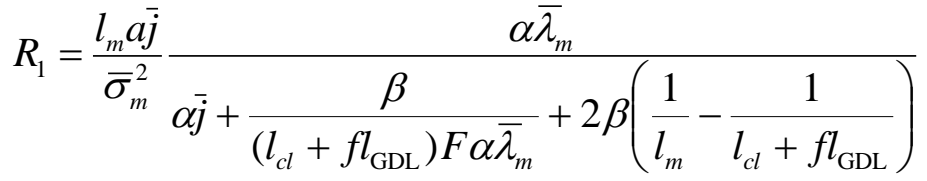




$$
\begin{aligned}
& R_{2}=\frac{l_{m} a \bar{j}}{\bar{\sigma}_{m}^{2}} \frac{\alpha \bar{\lambda}_{m}}{\frac{2 \beta}{l_{c l}+f l_{\mathrm{GDL}}}\left(1-\frac{1}{2 F \alpha \bar{\lambda}_{m}}\right)-\frac{\alpha \bar{j}+\frac{2 \beta}{l_{m}}}{1-\frac{D_{\mathrm{GDL}}^{w} \eta_{c h} F \rho_{m} l_{m} \alpha \bar{\lambda}_{m}}{l_{\mathrm{GDL}} \beta M_{m}}}} \\
& L=\frac{l_{m} a \bar{j}}{\bar{\sigma}_{m}^{2}} \frac{\frac{\alpha^{2} \bar{\lambda}_{m}^{2} l_{m} F\left(l_{c l}+f l_{\mathrm{GDL}}\right) \rho_{m}}{\beta M_{m}}}{\left(\alpha \bar{j}+\frac{2 \beta}{l_{m}}\right)-\frac{2 \beta}{l_{c l}+f l_{\mathrm{GDL}}}\left(1-\frac{1}{2 F \alpha \bar{\lambda}_{m}}\right)\left(1-\frac{D_{\mathrm{GDL}}^{w} \eta_{c h} F \rho_{m} l_{m} \alpha \bar{\lambda}_{m}}{l_{\mathrm{GDL}} \beta M_{m}}\right)}
\end{aligned}
$$

\subsection{Results and discussions}

In this section, the presented equivalent circuit and the elements are compared against the measured fuel cell Nyquist plots presented in literature $[20,46,91]$. The results are shown in Figures 2.2, 2.3 and 2.4. Since this chapter focuses on the ohmic loss, the comparisons are conducted between the low frequency (LF) arcs of the experiments and those obtained through the model. The complete model (including the ohmic loss along with the activation and mass transport losses) is compared against the impedances measured in our lab and the results are analyzed in Chapter 4.

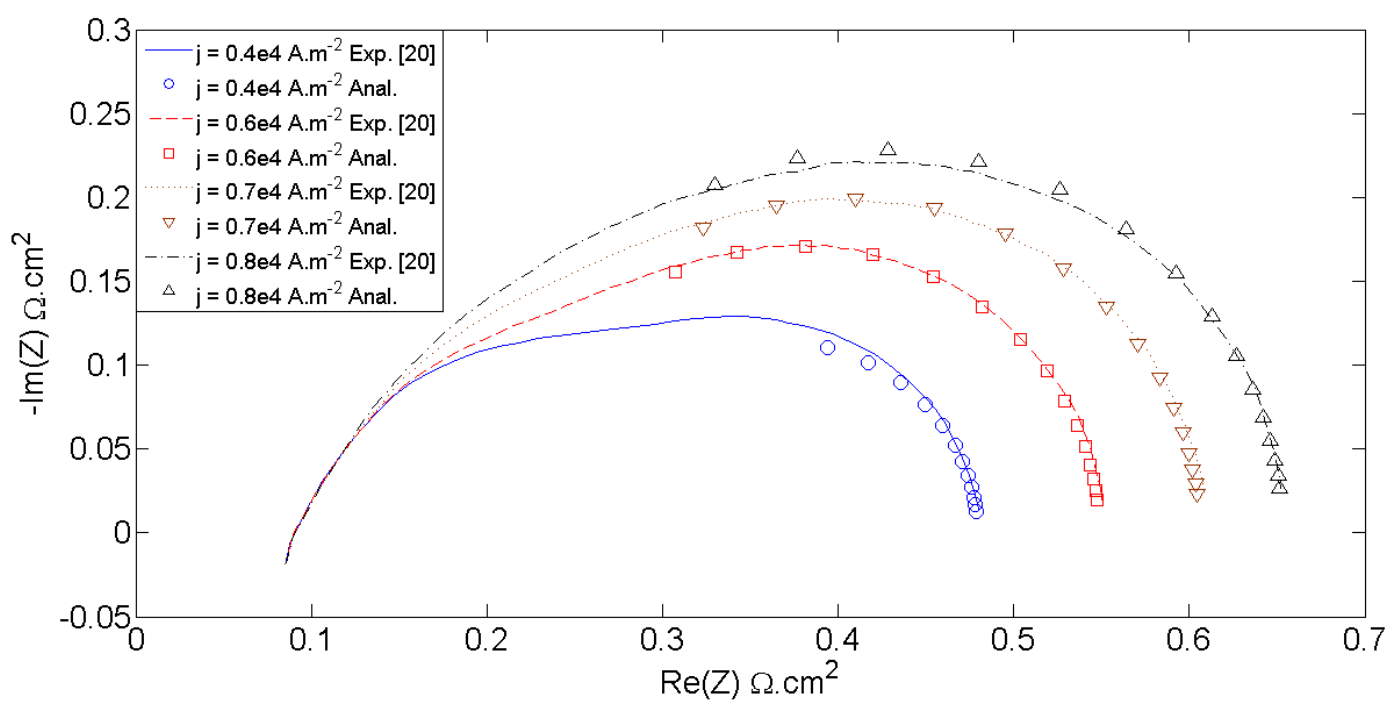

Figure 2.2 Comparison between the curves obtained from the ohmic loss model and the measured impedances measured by Ref. [20] 
Danzer and Hofer [20] measured the impedances of a five-cell stack with an active surface area of $100 \mathrm{~cm}^{2}$ at the cell temperature of $55^{\circ} \mathrm{C}$. The SGL 10BB was used as the gas diffusion layer. The measurements were conducted within the frequency range of $0.2 \mathrm{~Hz}$ to $10 \mathrm{kHz}$ and at the current densities of $0.4 \mathrm{~A} \cdot \mathrm{cm}^{-2}, 0.6 \mathrm{~A} \cdot \mathrm{cm}^{-2}, 0.7 \mathrm{~A} \cdot \mathrm{cm}^{-2}$ and $0.8 \mathrm{~A} \cdot \mathrm{cm}^{-2}$. Figure 2.2 compares their measured Nyquist plots with those obtained through the analytical model. The model predictions are in great agreement with the measured impedances (with the discrepancy less than $2 \%$ ). Malevich et al. [46] reported the Nyquist plots for the frequency range of $0.1 \mathrm{~Hz}-60 \mathrm{kHz}$. The cell contained Nafion 112 with a $35-\mu \mathrm{m}$ catalyst layer and SGL, and was operated at $60{ }^{\circ} \mathrm{C}$. Figure 2.3 compares the predictions from the analytical model and the measured impedances in the low frequency range. The arc diameter and the time constants obtained from the theoretical and experimental curves are in great agreement (with the discrepancy less than $2 \%$ ). Finally, the measured impedances of a $26-\mathrm{cm}^{2}$ cell operated at temperature of $70{ }^{\circ} \mathrm{C}$ [91] are compared against the results from the model (see Figure 2.4). The cell contained Nafion 1035 and Toray TGP-H-060. The measurements were conducted within the frequency range of $10 \mathrm{mHz}$ to $1 \mathrm{kHz}$ (with 5 points per decade) for the current densities of $0.231 \mathrm{~A} . \mathrm{cm}^{-2}$ and $0.346 \mathrm{~A} . \mathrm{cm}^{-2}$. As the measurements cover low frequencies, the inductive loop captured in the measured impedances is in good agreement with the model in terms of the general trend, arc diameters and time constants (with the discrepancy less than 5\%).

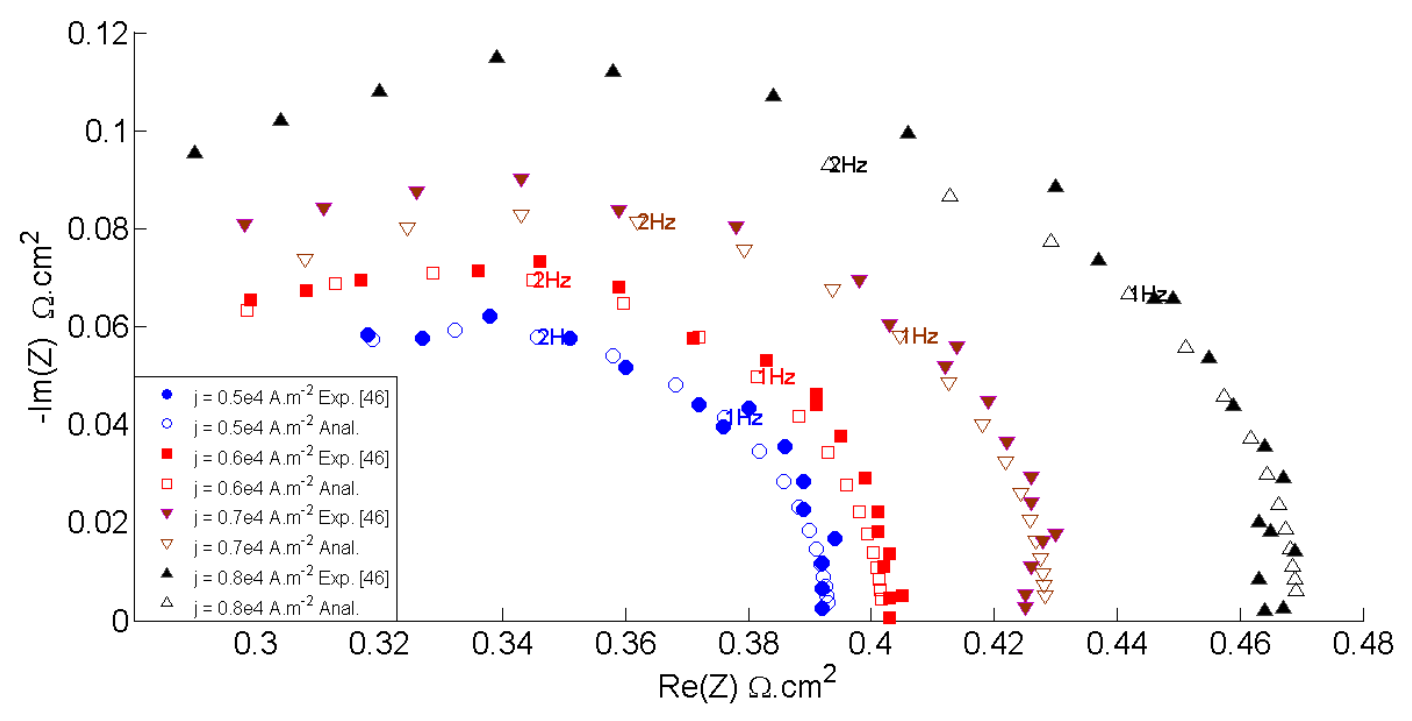

Figure 2.3 Comparison between the points obtained from the analytical model and the measured impedances (measured by [46]) in the low frequency range 


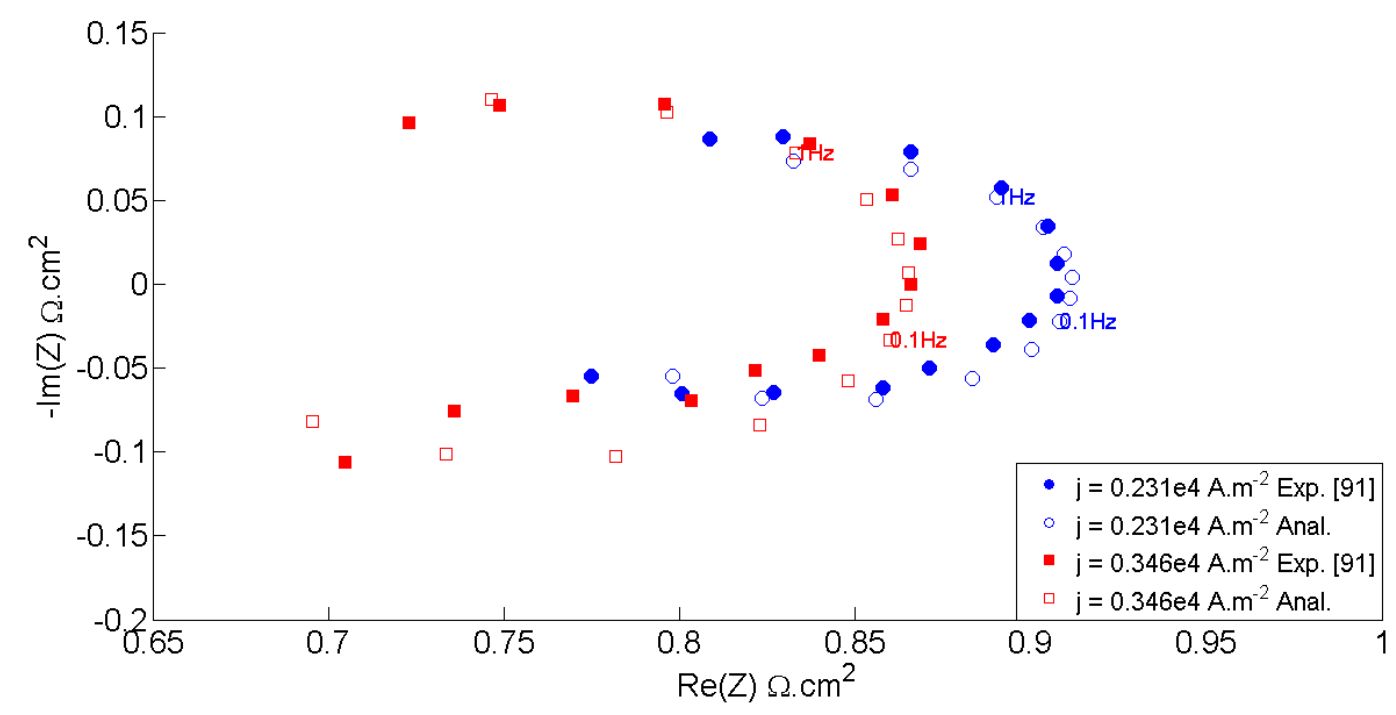

Figure 2.4 Comparison between the points obtained from the analytical model and the measured impedances (measured by [91]) in the low frequency range

\subsection{Concluding remarks}

The process modeling of the ohmic overpotential in the proton exchange membrane (PEM) fuel cells was discussed in this chapter. The impedance of the PEM fuel cell was divided into the impedance of the ohmic, activation and mass transport overpotentials as the main losses of the cell. Then, the impedance of the ohmic loss was analytically determined using the water transport equations for the membrane and the cathode catalyst and gas diffusion layers. Next, the impedance relation was converted into an equivalent circuit. Finally, the predicted impedance was compared against the measured impedances of the cells reported in literature. The agreement between the measured and predicted impedances for different operating conditions was excellent. 


\section{Chapter 3. Process modeling of electrodes ${ }^{1}$}

The impedance characteristics of the activation and mass transport overpotentials in a proton exchange membrane fuel cell are determined analytically using a process model. The anode and cathode electrodes are studied separately. The governing equations are solved analytically and the resultant impedances and hence the equivalent circuits are extracted. It is shown that the high frequency arc is the result of the anode impedance and is not a function of the current density. On the other hand, the mid-frequency arc consisting of two convoluted depressed semi-circles is the result of the cathode impedance. The solution clarifies the reason for an initial decrease followed by an increase in the diameter of the mid-frequency arc as the current density increases. The results also show that cathode starvation can be studied clearly from the mid frequency arc, while the anode starvation cannot be distinguished from the impedance measurements.

\subsection{Anode impedance}

Figure 3.1 shows the schematic view of the anode-membrane interphase. The hydrogen gas $\left(\mathrm{H}_{2}\right)$ is diffused through the anode gas diffusion layer and adsorbed on an empty site (A) at the Pt surface and dissociated into the hydrogen atoms $\left(\mathrm{H}_{\mathrm{ads}}\right)$ in a rapid reaction [134]

$1 / 2 \mathrm{H}_{2}+\mathrm{A} \rightarrow \mathrm{H}_{\mathrm{ads}}$

The adsorbed hydrogen atom is then discharged into a proton $\left(\mathrm{H}^{+}\right)$and electron $\left(\mathrm{e}^{-}\right)$according to the following electrochemical reaction:

$\mathrm{H}_{\mathrm{ads}} \rightarrow \mathrm{H}^{+}+\mathrm{e}^{-}$

${ }^{1}$ Parts of Chapter 3 have been published in a journal article. Reprinted with permission from [203]. 


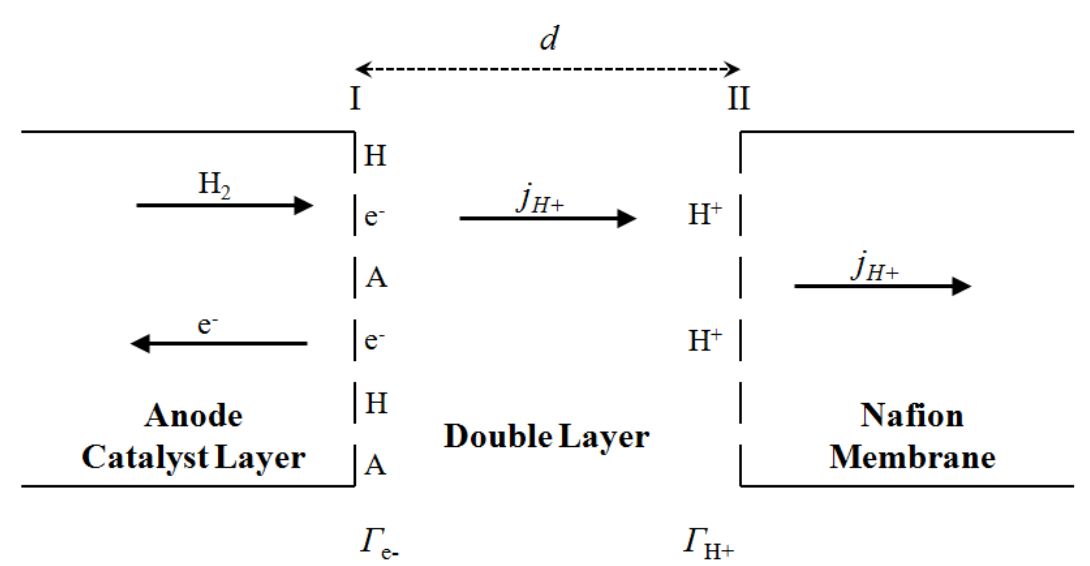

Figure 3.1 The schematic view of the anode-membrane interphase

The proton $\left(\mathrm{H}^{+}\right)$is then displaced to the Nafion surface (II) and causes a surplus surface density of a positive charge at the plane II $\left(\Gamma_{\mathrm{H}^{+}}\right)$. Similarly, the remaining electron causes a surplus surface density of a negative charge at the plane $\mathrm{I}\left(\Gamma_{\mathrm{e}^{-}}\right)$. These coupled charges are the origin of the formation of the double layer capacitance which occurs in a layer with a thickness of $d$ (with the order of magnitude of $1 \mathrm{~nm}$ [134]). The total resultant electrical current $\left(j_{t o t}\right)$ presented in reaction (3.2) is the summation of the proton transfer current $\left(j_{H^{+}}\right)$and the displacement current [135]

$j_{t o t}=j_{\mathrm{H}^{+}}+\varepsilon_{s} \frac{\partial E_{s}}{\partial t}$

where $\varepsilon_{s}$ and $E_{s}$ are the dielectric permittivity and the electric field strength at the interphase, respectively. On the other hand, the anode overpotential $\left(\eta_{A}\right)$ can be written as [134]

$\eta_{A}=\varphi_{\mathrm{I}}-\varphi_{\mathrm{II}}-\mu_{\mathrm{H}^{+}} / F$

where $\varphi_{\mathrm{I}}, \varphi_{\mathrm{II}}$, and $F$ are the electric potentials in the layers I and II, and the Faraday constant (96485 C. $\left.\mathrm{mol}^{-1}[2]\right)$, respectively. $\mu_{\mathrm{H}^{+}}$is the chemical potential of transferring protons $[2,134]$. The electric potential difference $\left(\varphi_{\mathrm{I}}-\varphi_{\text {II }}\right)$ can be determined from the electric field strength $\left(E_{s}\right)$ using the parallel plate assumption for the plates I and II (this parallel plate assumption is revisited in the discussions related to the constant phase element (CPE))

$\varphi_{\mathrm{I}}-\varphi_{\mathrm{II}}=d \cdot E_{s}$ 
where $d$ is the thickness of the so-called interphase layer (Figure 3.1). Also, the chemical potential can be determined as [2]

$$
\mu_{\mathrm{H}^{+}}=\mu_{\mathrm{H}^{+}}^{0}+R T \ln \frac{\Gamma_{\mathrm{H}^{+}}}{\Gamma_{\mathrm{H}^{+}}^{0}}
$$

where $\mu_{\mathrm{H}^{+}}^{0}, R, T, \Gamma_{\mathrm{H}^{+}}$and $\Gamma_{\mathrm{H}^{+}}^{0}$ present the reference chemical potential at the standard state condition, the ideal gas constant $\left(8.314 \mathrm{~J} \cdot \mathrm{mol}^{-1} \cdot \mathrm{K}^{-1}[2]\right)$, temperature, the surface density of the protons at the plane II, and the surface density of the protons at the standard state condition, respectively. Neglecting the electric field in the membrane [134], the surface density of the protons at the plane II $\left(\Gamma_{\mathrm{H}^{+}}\right)$can be written as

$$
\Gamma_{\mathrm{H}^{+}}=-\varepsilon_{s} E_{s}
$$

It has to be mentioned that the above assumption (i.e., neglecting the electric field in the membrane) will be revisited in the discussions related to the constant phase element (CPE).

To specify the impedance characteristics of the electrode, the parameters are perturbed and the impedance is calculated. The perturbed parts of the parameters are presented with the symbol , e.g., $\tilde{\Gamma}_{\mathrm{H}^{+}}$; while the steady-state parts which show the values of the parameters prior to perturbation are shown with the symbol ${ }^{-}$, e.g., $\bar{\mu}_{\mathrm{H}^{+}}$. Using these notations, Equation (3.7) can be represented as

$$
\bar{\Gamma}_{\mathrm{H}^{+}}+\tilde{\Gamma}_{\mathrm{H}^{+}}=-\varepsilon_{s}\left(\bar{E}_{s}+\tilde{E}_{s}\right)
$$

Considering the validity of Equation (3.7) in the steady-state condition $\left(\bar{\Gamma}_{\mathrm{H}^{+}}=-\varepsilon_{s} \bar{E}_{s}\right)$, the perturbed part of the proton surface density $\left(\tilde{\Gamma}_{\mathrm{H}^{+}}\right)$can be determined as

$$
\tilde{\Gamma}_{\mathrm{H}^{+}}=-\varepsilon_{s} \tilde{E}_{s}
$$

Based on the assumption of a small perturbation, the perturbed parts of the above-mentioned parameters from Equations (3.3)-(3.6) can be determined as

$$
\begin{aligned}
& \tilde{j}_{t o t}=\tilde{j}_{\mathrm{H}^{+}}+\varepsilon_{s}(i \omega) \tilde{E}_{s} \\
& \tilde{\eta}_{A}=\tilde{\varphi}_{\mathrm{I}}-\tilde{\varphi}_{\mathrm{II}}-\tilde{\mu}_{\mathrm{H}^{+}} / F \\
& \tilde{\varphi}_{\mathrm{I}}-\tilde{\varphi}_{\mathrm{II}}=d \cdot \tilde{E}_{s}
\end{aligned}
$$


$\tilde{\mu}_{\mathrm{H}^{+}}=R T \frac{\tilde{\Gamma}_{\mathrm{H}^{+}}}{\bar{\Gamma}_{\mathrm{H}^{+}}}$

where $\omega$ is the angular velocity and $i=\sqrt{-1}$. Using Equations (3.9)-(3.13), one can find

$\tilde{\eta}_{A}=\left(d+\frac{R T \varepsilon_{s}}{F \bar{\Gamma}_{\mathrm{H}^{+}}}\right) \tilde{E}_{s}$

The anode electrode impedance $\left(Z_{A}\right)$ can then be determined as

$Z_{A}=\frac{\tilde{\eta}_{A}}{\widetilde{j}_{t o t}}=\left(d+\frac{R T \varepsilon_{s}}{F \bar{\Gamma}_{\mathrm{H}^{+}}}\right) \frac{\widetilde{E}_{s}}{\widetilde{\tilde{j}}_{t o t}}$

The electrical current $\left(j_{\mathrm{H}^{+}}\right)$can be determined from the Butler-Volmer equation [2]

$j_{\mathrm{H}^{+}}=j_{0, A}\left(\frac{c_{\mathrm{H}_{2}}}{c_{\mathrm{H}_{2}}^{0}} \exp \left(\frac{\alpha_{A} n F \eta_{A}}{R T}\right)-\left(\frac{c_{\mathrm{H}^{+}, s}}{c_{\mathrm{H}^{+}, \mathrm{s}}^{0}}\right)^{2} \exp \left(\frac{-\left(1-\alpha_{A}\right) n F \eta_{A}}{R T}\right)\right)$

where $j_{0, A}, \alpha_{A}$ and $n$ are the anode exchange current density, the anode transfer coefficient and the number of electrons transferred due to reaction $(n=2)$, respectively. Also, $c_{\mathrm{H}_{2}}, c_{\mathrm{H}^{+}, \mathrm{s}}, c_{\mathrm{H}_{2}}^{0}$ and $c_{\mathrm{H}^{+}, \mathrm{s}}^{0}$ are the actual hydrogen gas concentration, the actual adsorbed hydrogen atom concentration, and their reference values, respectively [2] (see Section 3.3). It has been shown that the assumption of a very low overpotential for the anode of the PEM fuel cell is highly accurate in the entire range of fuel cell performance $[136,137]$. Considering the value of $\alpha_{A}=0.5[138-140]$ and assuming the temperature of $T=60{ }^{\circ} \mathrm{C}(333 \mathrm{~K})$, the $\frac{\alpha_{A} n F}{R T}$ term will be around $35 \mathrm{~V}^{-1}$. The anode to cathode activation overpotential ratio is stated to be around 1/2000 in the literature [141] (see Section 3.3). If this ratio becomes even considerably larger in practice (even as large as 1/100) and the activation overpotential even becomes $1 \mathrm{~V}$ in worst cases, then the anode overpotential will still be less than $0.01 \mathrm{~V}$ which produces an error of less than $2 \%$ due to the linearization of Butler-Volmer equation [136]. This verifies that we can linearize (with a minimum error) the exponential terms.

$j_{\mathrm{H}^{+}}=j_{0, A}\left(\left(\frac{c_{\mathrm{H}_{2}}}{c_{\mathrm{H}_{2}}^{0}}-\left(\frac{c_{\mathrm{H}^{+}, s}}{c_{\mathrm{H}^{+}, \mathrm{s}}^{0}}\right)^{2}\right)\left(1+\frac{\alpha_{A} n F \eta_{A}}{R T}\right)+\left(\frac{c_{\mathrm{H}^{+}, s}}{c_{\mathrm{H}^{+}, \mathrm{s}}^{0}}\right)^{2} \frac{n F \eta_{A}}{R T}\right)$ 
The term of $\left(\frac{c_{\mathrm{H}_{2}}}{c_{\mathrm{H}_{2}}^{0}}-\left(\frac{c_{\mathrm{H}^{+}, s}}{c_{\mathrm{H}^{+}, \mathrm{s}}^{0}}\right)^{2}\right)$ can be neglected (see the discussions in section 3.3). Therefore, the electrical current can be simplified as

$$
j_{\mathrm{H}^{+}}=j_{0, A}\left(\frac{c_{\mathrm{H}^{+}, s}}{c_{\mathrm{H}^{+}, \mathrm{s}}^{0}}\right)^{2} \frac{n F \eta_{A}}{R T}
$$

The perturbed electrical current can then be determined as

$$
\tilde{j}_{\mathrm{H}^{+}}=j_{0, A}\left(\frac{\bar{c}_{\mathrm{H}^{+}, s}}{c_{\mathrm{H}^{+}, \mathrm{s}}^{0}}\right)^{2} \frac{n F}{R T} \tilde{\eta}_{A}+j_{0, A} \frac{2 \bar{c}_{\mathrm{H}^{+}, s}}{c_{\mathrm{H}^{+}, \mathrm{s}}^{0}} \frac{n F \bar{\eta}_{A}}{R T} \tilde{c}_{\mathrm{H}^{+}, s}
$$

Assuming an infinite rate of reaction for hydrogen adsorption (Equation (3.1)) [134], the concentration of the adsorbed hydrogen atom can be estimated from the electrical current and the hydrogen diffusion rate.

$$
\frac{\partial c_{\mathrm{H}^{+} \mathrm{s}}}{\partial t}=-\frac{j_{\mathrm{H}^{+}}}{F}+2 D_{\mathrm{GDL}, \mathrm{A}} \frac{\partial c_{\mathrm{H}_{2}}}{\partial x}
$$

where $D_{\mathrm{GDL}, \mathrm{A}}$ and $c_{\mathrm{H}_{2}}$ present the anode gas diffusion layer (GDL) diffusivity and the concentration of the adsorbed hydrogen gas, respectively. In the above equation, the first term on the right hand side depicts the proton consumption rate in the cathode which is equivalent to the proton transfer rate from the anode catalyst layer towards the membrane. The second term determines the hydrogen diffusion rate from the anode GDL to the anode catalyst layer. While the diffusion term models the diffusion in the GDL, the diffusion coefficient can be considered as the "effective" diffusion coefficient covering also the gas diffusion in the catalyst layer. Clearly in this case, this effective diffusion coefficient has to be corrected based on the porous structures of the catalyst and gas diffusion layers. It also has to be remembered that $c_{\mathrm{H}^{+}, \mathrm{s}}$, the parameter in the left hand side of Equation (3.20), is a surface concentration (with the unit of $\mathrm{mol} \cdot \mathrm{m}^{-2}$ ), while the $c_{\mathrm{H}_{2}}$ parameter (in the right hand side) is a volumetric concentration (with the unit of $\left.\mathrm{mol} \cdot \mathrm{m}^{-3}\right)$. Finally, it has to be emphasized that the diffusion term in the above equation is clearly different than the diffusion term used by Randles [21], as he solved the reaction on a micro-electrode in an aqueous solution which is fundamentally different from the gas diffusion in the fuel cell electrodes. 
By estimating the concentration gradient of the adsorbed hydrogen gas based on the concentrations of the hydrogen gas in the anode channel $\left(c_{\mathrm{H}_{2}, \mathrm{ch}}\right)$ and catalyst layer $\left(c_{\mathrm{H}_{2}, \mathrm{cl}}\right)$ $\left(\frac{\partial c_{\mathrm{H}_{2}}}{\partial x}=\frac{c_{\mathrm{H}_{2}, \mathrm{ch}}-c_{\mathrm{H}_{2}, \mathrm{cl}}}{l_{\mathrm{GDL}, \mathrm{A}}}\right.$, where $l_{\mathrm{GDL}, \mathrm{A}}$ presents the thickness of the GDL in the anode $)$ and also assuming the infinite rate of reaction for hydrogen adsorption for determining the concentration of the hydrogen gas at the catalyst layer $\left(c_{\mathrm{H}_{2}, \mathrm{cl}}=\frac{1}{2} \frac{c_{\mathrm{H}^{+}, \mathrm{s}}}{l_{\mathrm{CL}, \mathrm{A}}}\right.$, where $l_{\mathrm{CL}, \mathrm{A}}$ presents the thickness of the catalyst layer in the anode), the perturbed part of the concentration of the adsorbed hydrogen atom can be calculated based on

$$
\tilde{c}_{\mathrm{H}^{+}, \mathrm{s}}=\frac{-\tilde{j}_{\mathrm{H}^{+}}}{F\left(i \omega+\frac{D_{\mathrm{GDL}, \mathrm{A}}}{l_{\mathrm{GDL}, \mathrm{A}} l_{\mathrm{CL}, \mathrm{A}}}\right)}
$$

Combining Equations (3.19) and (3.21), one can find

$$
\left(1+j_{0, A} \frac{2 \bar{c}_{\mathrm{H}^{+}, s}}{c_{\mathrm{H}^{+}, \mathrm{s}}^{0}{ }^{2}} \frac{n F \bar{\eta}_{\mathrm{A}} /(R T)}{F\left(i \omega+\frac{D_{\mathrm{GDL}, \mathrm{A}}}{l_{\mathrm{GDL}, \mathrm{A}} l_{\mathrm{CL}, \mathrm{A}}}\right)}\right) \tilde{j}_{\mathrm{H}^{+}}=j_{0, A}\left(\frac{\bar{c}_{\mathrm{H}^{+}, s}}{c_{\mathrm{H}^{+}, \mathrm{s}}^{0}}\right)^{2} \frac{n F}{R T} \tilde{\eta}_{A}
$$

Using Equations (3.10), (3.15) and (3.22), it can be shown that

$$
1=\frac{j_{0, A}\left(\frac{\bar{c}_{\mathrm{H}^{+}, s}}{c_{\mathrm{H}^{+}, \mathrm{s}}^{0}}\right)^{2} \frac{n F}{R T}}{1+j_{0, A} \frac{2 \bar{c}_{\mathrm{H}^{+}, s}}{c_{\mathrm{H}^{+}, \mathrm{s}}^{0}{ }^{2}} \frac{n F \bar{\eta}_{A} /(R T)}{F\left(i \omega+\frac{D_{\mathrm{GDL}, \mathrm{A}}}{l_{\mathrm{GDL}, \mathrm{A}} l_{\mathrm{CL}, \mathrm{A}}}\right)}} Z_{A}+\varepsilon_{s}(i \omega) \frac{1}{d+\frac{R T \varepsilon_{s}}{F \bar{\Gamma}_{\mathrm{H}^{+}}}} Z_{A}
$$

Finally, the anode electrode impedance can be determined as

$$
Z_{A}=\frac{\frac{i \omega+B_{A}+C_{A}}{A_{A} i \omega+A_{A} C_{A}}}{1+D_{A} i \omega\left(\frac{i \omega+B_{A}+C_{A}}{A_{A} i \omega+A_{A} C_{A}}\right)}
$$

where 


$$
\begin{aligned}
& A_{A}=j_{0, A}\left(\frac{\overline{\mathrm{H}}_{\mathrm{H}^{+}, s}}{c_{\mathrm{H}^{+}, \mathrm{s}}^{0}}\right)^{2} \frac{n F}{R T} \\
& B_{A}=j_{0, A} \frac{2 \bar{c}_{\mathrm{H}^{+}, s}}{c_{\mathrm{H}^{+}, \mathrm{s}}^{0}} \frac{n \bar{\eta}_{A}}{R T} \\
& C_{A}=\frac{D_{\mathrm{GDL}, \mathrm{A}}}{l_{\mathrm{GDL}, \mathrm{A}} l_{\mathrm{CL}, \mathrm{A}}} \\
& D_{A}=\frac{\varepsilon_{s}}{d+\frac{R T \varepsilon_{s}}{F \bar{\Gamma}_{\mathrm{H}^{+}}}}
\end{aligned}
$$

The impedance in Equation (3.24) is the impedance of a circuit consisting of a capacitance $D_{A}$ (double-layer capacitance) which is in parallel with a block of $Z_{p, A}$ presented as

$$
Z_{p, A}=\frac{i \omega+B_{A}+C_{A}}{A_{A} i \omega+A_{A} C_{A}}
$$

The effects of the porous structure of the electrode are studied in the next steps by replacing the double-layer capacitance with the constant phase element (CPE). However, there is an important point regarding the parallel plate assumption made for the electrode: the double-layer element is in parallel connection to the remaining block of the elements represented by $Z_{p, A}$ (Equation

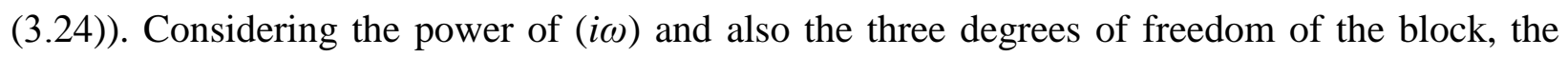
equivalent impedance of $Z_{p, A}$ can be obtained from a circuit containing a parallel-connected resistance and capacitance which are connected to another resistance in series. Therefore, the complete anode equivalent circuit can be presented as the combination of the elements shown in Figure 3.2.a. These elements can be obtained as

$$
\begin{aligned}
& C_{d l, A}=D_{A}=\frac{\varepsilon_{s}}{d+\frac{R T \varepsilon_{s}}{F \bar{\Gamma}_{\mathrm{H}^{+}}}} \\
& R_{1, A}=\frac{1}{A_{A}}=\frac{1}{j_{0, A}}\left(\frac{c_{\mathrm{H}^{+}, \mathrm{s}}^{0}}{\bar{c}_{\mathrm{H}^{+}, s}}\right)^{2} \frac{R T}{n F} \\
& R_{2, A}=\frac{B_{A}}{A_{A} C_{A}}=\frac{l_{\mathrm{GDL}, \mathrm{A}} l_{\mathrm{CL}, \mathrm{A}}}{D_{\mathrm{GDL}, \mathrm{A}}} \frac{R T}{n F^{2}}\left(\frac{c_{\mathrm{H}^{+}, \mathrm{s}}^{0}}{\bar{c}_{\mathrm{H}^{+}, s}}\right)^{3} \frac{2 \bar{j}}{j_{0, A}}
\end{aligned}
$$




$$
C_{2, A}=\frac{A_{A}}{B_{A}}=\frac{n F^{2}}{R T}\left(\frac{\bar{c}_{\mathrm{H}^{+}, s}}{c_{\mathrm{H}^{+}, \mathrm{s}}^{0}}\right)^{3} \frac{j_{0, A}}{2 \bar{j}}
$$

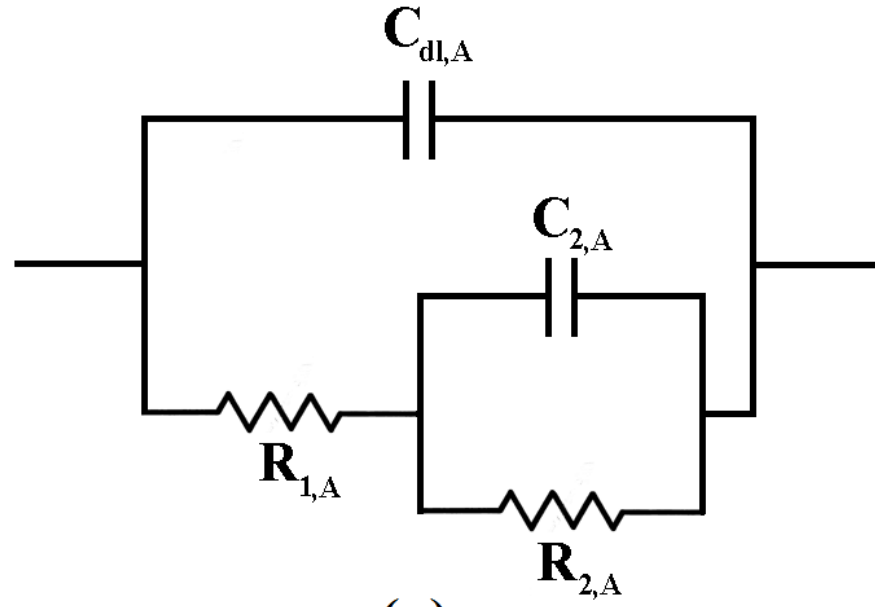

(a)

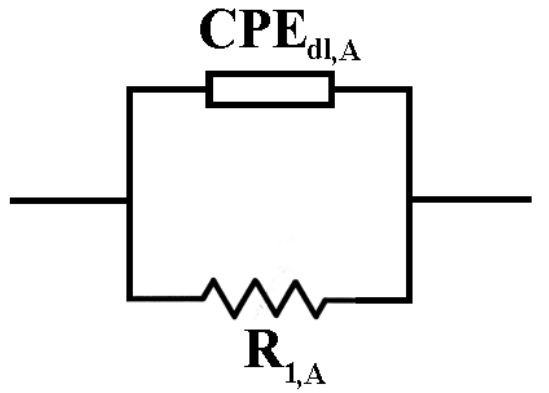

(b)

Figure 3.2 The PEM fuel cell anode equivalent circuit; (a) the complete version, (b) the simplified version

To compare this model with the measured impedances and estimate the values of the presented elements, the values of the parameters presented in Table 3.1 are considered. Based on the value of hydrogen gas diffusivity [142] and the corrections required for compensating the effect of the characteristics of the porous media on diffusion [143, 144], the anode GDL diffusivity is assumed as $1 \mathrm{e}-5 \mathrm{~m}^{2} \cdot \mathrm{s}^{-1}$. The values reported for the anode exchange current density $\left(j_{0, A}\right)$ in literature are considerably diverse [138, 145-154]. Although the values in the order of 1e-3 $\mathrm{mA} \cdot \mathrm{cm}^{-2}$ (mostly, $1.35 \mathrm{e}-3 \mathrm{~mA} \cdot \mathrm{cm}^{-2}$ ) were repeatedly reported [138, 145, 147, 148, 151-154], Gasteiger et al. [149, 150] stated that the correct value would be considerably bigger (in some cases, even up to $600 \mathrm{~mA} \cdot \mathrm{cm}^{-2}$ ) if the ohmic and mass transport losses are considered. There are also reported values which lie between these two limits [2, 146]. In essence, the exchange current density is proportional to the electrode's real surface area [155], and hence depends on the characteristics of the porous structure of the electrode. Here, the anode exchange current density is considered to be in the range of $100-300 \mathrm{~mA} \cdot \mathrm{cm}^{-2}$ and is obtained for each case presented in the verification section (Section 3.4) based on the best fit. The anode double layer capacitance is assumed to be $20 \mathrm{~F} \cdot \mathrm{m}^{-2}$ which has been obtained based on the correction factor 
[156-159] used to compensate for the effect of the surface roughness of the electrode (see the detailed discussion in the following paragraph). The proton concentration ratio of $\bar{c}_{\mathrm{H}^{+}, s} / c_{\mathrm{H}^{+}, \mathrm{s}}^{0}$ is assumed to be 1.0 (see Section 3.3).

Table 3.1 Parameters used for calculating the elements of the anode equivalent circuit

\begin{tabular}{llcr} 
Property & Variable & Dimension & Value \\
\hline Anode GDL thickness & $l_{\mathrm{GDL}, \mathrm{A}}$ & $\mathrm{m}$ & $4 \mathrm{e}-4$ \\
\hline Anode CL thickness & $l_{\mathrm{CL}, \mathrm{A}}$ & $\mathrm{m}$ & $1 \mathrm{e}-5$ \\
\hline Anode GDL diffusivity & $D_{\mathrm{GDL}, \mathrm{A}}$ & $\mathrm{m}^{2} \mathrm{~s}^{-1}$ & $1 \mathrm{e}-5$ \\
\hline Anode exchange current density & $j_{0, A}$ & $\mathrm{~A} \mathrm{~m}^{-2}$ & 1350 \\
\hline Current density & $\bar{j}$ & $\mathrm{~A} \mathrm{~m}^{-2}$ & $0.3 \mathrm{e} 4$ \\
\hline Anode double layer capacitance & $C_{d l, A}$ & $\mathrm{~F} \mathrm{~m}^{-2}$ & 20 \\
\hline Proton concentration ratio & $\bar{c}_{\mathrm{H}+, \mathrm{s}} / c_{\mathrm{H}+\mathrm{s}}^{0}$ & - & 1 \\
\hline Temperature & $T$ & ${ }^{\circ} \mathrm{C}$ & 60
\end{tabular}

Considering the parameters presented in Table 3.1, one can show that the value of the resistance $R_{2, A}$ is six orders of magnitude smaller than the value of $R_{1, A}$. Also, the value of the capacitance $C_{2, A}$ is five orders of magnitude larger than that of the double layer capacitance. Thus, the loop of $R_{2, A}-C_{2, A}$ can be neglected, resulting in the final equivalent circuit presented in Figure 3.2.b. As it can be seen in this figure, the double layer capacitance is also replaced with a constant phase element (CPE) (as suggested in [155]) which has the impedance in the form

$Z_{\mathrm{CPE}, A}=\frac{1}{C_{d l, A}(i \omega)^{n_{\mathrm{CPE}, A}}}$

where $n_{C P E, A}$ is the CPE exponent. As it has been discussed thoroughly in the literature [155], the porous solid electrodes contradict the parallel-plate assumption employed here in two ways: first, due to the porous structure, the real surface area is considerably larger than the superficial surface area of the electrode. As a result, the double layer capacitance value is normally corrected using the roughness factor obtained based on the ratio of the real to the superficial surface area $[118,160,161]$. Second, the porous electrode causes the related semi-circle in the measured Nyquist plots to be depressed which was practically best fitted using the above- 
mentioned constant phase element (CPE) [162]. Although there is no well-justified microscopic theory behind CPEs [155], there were attempts towards relating the CPE power ( $n_{C P E}$ ) to physicochemical properties of the electrode (e.g., the fractal dimension of the electrode [163]) which are still debatable $[155,164]$. It was recently shown that the CPE could be the result of different time constants for the processes happening in a porous electrode [165-167]. However, this still does not present the physical interpretation for the element. As all the parameters related to the parallel plate assumption (i.e., the dielectric permittivity at the interphase $\left(\varepsilon_{s}\right)$, the double layer thickness $(d)$, and the steady-state part of the proton surface density $\left(\bar{\Gamma}_{\mathrm{H}^{+}}\right)$) are only appeared in the double layer capacitance (Equation (3.30)), this is the only element related to the so-called parallel plate assumption, and as a result, it can be replaced with the CPE without affecting the analysis related to the Butler-Volmer equation (Equation (3.29)) as discussed before in literature $[10,155]$. It has to be mentioned that the assumption of neglecting the electric field in the membrane is also removed as the resultant double layer capacitance is finally replaced by a CPE.

Considering the parameters presented in Table 3.1, the Nyquist plot of the anode equivalent circuit is a semi-circle with the diameter of around $0.11 \Omega \cdot \mathrm{cm}^{2}$ and the peak frequency of around $1000 \mathrm{~Hz}$. The measured Nyquist plots reported in the PEM fuel cell [20, 34, 46, 114] show a semi-circle with the same diameter and time constant. This semi-circle is often referred to as the high frequency (HF) arc [34]. The impedance measurements reported in the literature demonstrate that the HF arc is the only semi-circle observed in the case of $\mathrm{H}_{2} / \mathrm{H}_{2}$ feeding (fuel cell operates with only $\mathrm{H}_{2}$ at both cathode and anode electrodes) [34] and do not change as the current density changes [20,46,114]. The presented equivalent circuit in Figure 3.2.b and the calculated elements (Equations (3.31) and (3.34)) confirm the same conclusions. As the HF arc comes from the anode impedance, it is reasonable to be the only arc captured in the case of $\mathrm{H}_{2} / \mathrm{H}_{2}$ feeding. Moreover, as the $R_{1, A}$ element value is independent of the current density $(\bar{j})$, the HF arc obtained based on the presented equivalent circuit is expected to be independent of the current density, as it is the case shown in the measured impedances.

The relation between the anode impedance and the HF arc can be questioned as the anode overpotential was reported negligible in literature $[34,141]$ (this assumption was also used here in the Butler-Volmer equation and deriving process of Equation (3.18)). However, the HF arc 
shows a considerable resistance which is not negligible compared to other parts of the Nyquist plot. This argument is thoroughly discussed in Section 3.3.

Finally, it has to be mentioned that anode starvation cannot be captured by the high frequency arc obtained from the equivalent circuit presented in Figure 3.2.b. In essence, one can suggest that anode starvation might be modeled via changing the anode GDL diffusion coefficient ( $\left.D_{\mathrm{GDL}, \mathrm{A}}\right)$, as a decrease in the diffusion coefficient results in a lower hydrogen concentration on the anode catalyst layer which can be presented as one mode of anode starvation. Regardless of the source of starvation (whether it is flooding or low diffusion coefficient), the final result would be a low hydrogen concentration on the catalyst layer which is equivalent to assuming a low GDL diffusion coefficient. In fact, the assumption of a low diffusion coefficient can indirectly model anode flooding effects. Even if this is accepted, the change of the anode GDL diffusion coefficient does not affect the predicted impedances as the related element in the anode equivalent circuit (i.e. $R_{2, A}$ ) is orders of magnitude smaller than $R_{1, A}$, and the $C_{2, A}$ capacitance is extremely large (its impedance is negligible) resulting in neglecting the loop of $R_{2, A}-C_{2, A}$ in the final equivalent circuit. Nevertheless, this discussion needs to be verified by conducting experiments in controlled conditions which will be presented and discussed in Chapter 6 .

\subsection{Cathode impedance}

Similar to the anode, the schematic view of the cathode-membrane interphase is shown in Figure 3.3. The oxygen gas is diffused through the cathode GDL and adsorbed on the surface of platinum $(\mathrm{Pt})$, producing water.

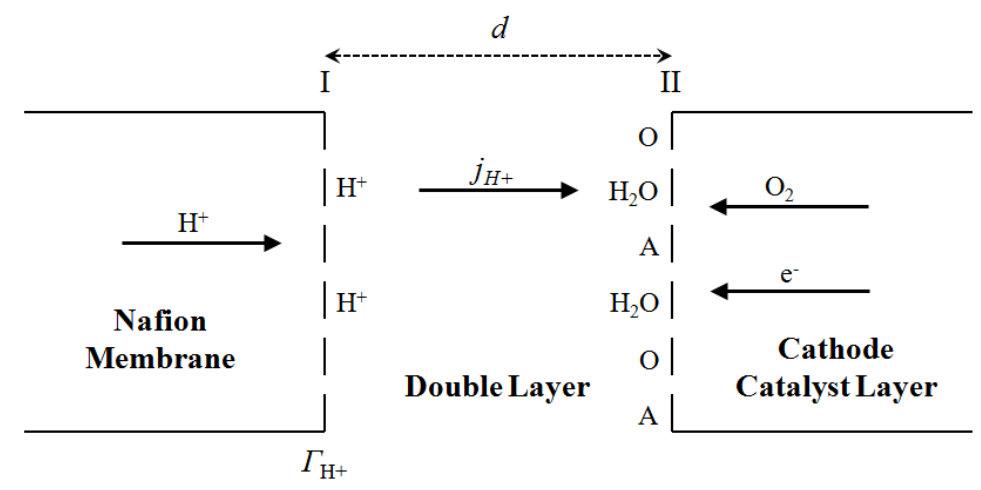

Figure 3.3 The schematic view of the cathode-membrane interphase 
$\mathrm{O}_{\mathrm{s}}+2 \mathrm{H}^{+}+2 \mathrm{e}^{-} \rightarrow \mathrm{H}_{2} \mathrm{O}$

Equations (3.3)-(3.13) can also be used for the cathode. Thus, the cathode electrode impedance $\left(Z_{C}\right)$ can be calculated as

$Z_{C}=\frac{\tilde{\eta}_{C}}{\widetilde{j}_{t o t}}=\left(d+\frac{R T \varepsilon_{s}}{F \bar{\Gamma}_{\mathrm{H}^{+}}}\right) \frac{\tilde{E}_{s}}{\widetilde{\tilde{j}}_{t o t}}$

The electrical current density $\left(j_{\mathrm{C}}\right)$ can be determined using the Butler-Volmer equation [2] for the cathode

$j_{\mathrm{C}}=j_{0, C}\left(\frac{c_{O, s}}{c_{O, s}^{0}}\left(\frac{c_{\mathrm{H}^{+}, s}}{c_{\mathrm{H}^{+}, s}^{0}}\right)^{2} \exp \left(\frac{\alpha_{C} n F \eta_{C}}{R T}\right)-\frac{c_{\mathrm{H}_{2} \mathrm{O}}}{c_{\mathrm{H}_{2} \mathrm{O}}^{0}} \exp \left(\frac{-\left(1-\alpha_{C}\right) n F \eta_{C}}{R T}\right)\right)$

where $c_{\mathrm{H}_{2} \mathrm{O}}, c_{O, s}, c_{\mathrm{H}_{2} \mathrm{O}}^{0}$ and $c_{O, s}^{0}$ are the actual water concentration, the actual adsorbed oxygen atom concentration, and their reference values, respectively. The Butler-Volmer equation can be numerically inversed using a non-linear least square method, and the cathode overpotential can be determined as a numerical function of the ratio of the current density to the exchange current density [136]. Using this method, it can be shown [136, 137] that the error of the Tafel approximation (neglecting the second term on the right hand side of the Equation (3.37)) for the current ratio of 10 is less than $0.3 \%$ which drastically drops as the current density increases. Here, the current ratio is defined as the ratio of the current density to the exchange current density (i.e., $j_{\mathrm{C}} / j_{0, C}$ ). Since the exchange current density of the cathode is very low $\left(j_{0, C}=\mathrm{O}(1 \mathrm{e}-9)\right)[168-172]$, regardless of the current density, the second term of the above equation can safely be neglected (since the current ratio will be much more than 10). Also, the ratio of $c_{\mathrm{H}^{+}, s} / c_{\mathrm{H}^{+}, \mathrm{s}}^{0}$ can be assumed as unity as the exchange current density of the proton formation in the anode electrode is at least seven orders of magnitude greater than the exchange current density of the cathode reaction. Therefore, the Butler-Volmer equation for the cathode can be simplified as

$$
j_{\mathrm{C}}=j_{0, C} \frac{c_{O, s}}{c_{O, s}^{0}} \exp \left(\frac{\alpha_{C} n F \eta_{C}}{R T}\right)
$$

The perturbed part of the current can then be determined as 


$$
\tilde{j}_{\mathrm{C}}=j_{0, C} \frac{\bar{c}_{O, s}}{c_{O, s}^{0}} \exp \left(\frac{\alpha_{C} n F \bar{\eta}_{C}}{R T}\right) \frac{\alpha_{C} n F}{R T} \tilde{\eta}_{C}+\frac{j_{0, C}}{c_{O, s}^{0}} \exp \left(\frac{\alpha_{C} n F \bar{\eta}_{C}}{R T}\right) \tilde{c}_{O, s}
$$

The adsorbed oxygen concentration can be determined by considering the electron consumption (current density) and the diffusion rate

$\frac{\partial c_{O, s}}{\partial t}=-\frac{j_{C}}{n F}+2 D_{\mathrm{GDL}, \mathrm{C}} \frac{\partial c_{\mathrm{O} 2}}{\partial x}$

The above equation shows that the concentration of the absorbed oxygen changes because of consumption (or current and water production) and the oxygen diffusion (in the cathode GDL) towards the cathode catalyst layer. Here again, $c_{O, s}$ parameter is a surface concentration (with the unit of $\mathrm{mol} \cdot \mathrm{m}^{-2}$ ), while the $c_{\mathrm{O}_{2}}$ parameter represents a volumetric concentration (with the unit of $\mathrm{mol} \cdot \mathrm{m}^{-3}$ ). Using the same method employed for the anode, one can find

$$
\tilde{c}_{O, s}=\frac{-\tilde{j}_{C}}{n F\left(i \omega+\frac{D_{\mathrm{GDL}, \mathrm{C}}}{l_{\mathrm{GDL}, \mathrm{C}} l_{\mathrm{CL}, \mathrm{C}}}\right)}
$$

Combining Equations (3.41) and (3.39), the following relation can be obtained

$$
\left(1+\frac{j_{0, C}}{c_{O, s}^{0}} \frac{\exp \left(\frac{\alpha_{C} n F \bar{\eta}_{C}}{R T}\right)}{n F\left(i \omega+\frac{D_{\mathrm{GDL}, \mathrm{C}}}{l_{\mathrm{GDL}, \mathrm{C}} l_{\mathrm{CL}, \mathrm{C}}}\right)}\right) \tilde{j}_{C}=j_{0, C} \frac{\bar{c}_{O, s}}{c_{O, s}^{0}} \exp \left(\frac{\alpha_{C} n F \bar{\eta}_{C}}{R T}\right) \frac{\alpha_{C} n F}{R T} \tilde{\eta}_{C}
$$

Considering the displacement current, the cathode impedance can be obtained from

$$
1=\frac{j_{0, C} \frac{\bar{c}_{O, s}}{c_{O, s}^{0}} \exp \left(\frac{\alpha_{C} n F \bar{\eta}_{C}}{R T}\right) \frac{\alpha_{C} n F}{R T}}{1+\frac{j_{0, C}}{c_{O, s}^{0}} \frac{\exp \left(\frac{\alpha_{C} n F \bar{\eta}_{C}}{R T}\right)}{n F\left(i \omega+\frac{D_{\mathrm{GDL}, \mathrm{C}}}{l_{\mathrm{GDL}, \mathrm{C}} l_{\mathrm{CL}, \mathrm{C}}}\right)}} Z_{C}+\varepsilon_{s}(i \omega) \frac{1}{d+\frac{R T \varepsilon_{s}}{F \bar{\Gamma}_{\mathrm{H}^{+}}}} Z_{C}
$$

Rearranging the above relation results in the following impedance for the cathode 


$$
Z_{C}=\frac{\frac{i \omega+B_{C}+C_{C}}{A_{C} i \omega+A_{C} C_{C}}}{1+D_{C} i \omega\left(\frac{i \omega+B_{C}+C_{C}}{A_{C} i \omega+A_{C} C_{C}}\right)}
$$

where

$$
\begin{aligned}
A_{C} & =j_{0, C} \frac{\bar{c}_{O, s}}{c_{O, s}^{0}} \exp \left(\frac{\alpha_{C} n F \bar{\eta}_{C}}{R T}\right) \frac{\alpha_{C} n F}{R T} \\
B_{C} & =\frac{j_{0, C}}{n F c_{O, s}^{0}} \exp \left(\frac{\alpha_{C} n F \bar{\eta}_{C}}{R T}\right) \\
C_{C} & =\frac{D_{\mathrm{GDL}, \mathrm{C}}}{l_{\mathrm{GDL}, \mathrm{C}} l_{\mathrm{CL}, \mathrm{C}}} \\
D_{C} & =\frac{\varepsilon_{s}}{d+\frac{R T \varepsilon_{s}}{F \bar{\Gamma}_{\mathrm{H}^{+}}}}
\end{aligned}
$$

As the cathode impedance has a similar format to that of the anode, the equivalent circuits expected to be similar (see Figure 3.4). However, the elements have different definitions, as presented below

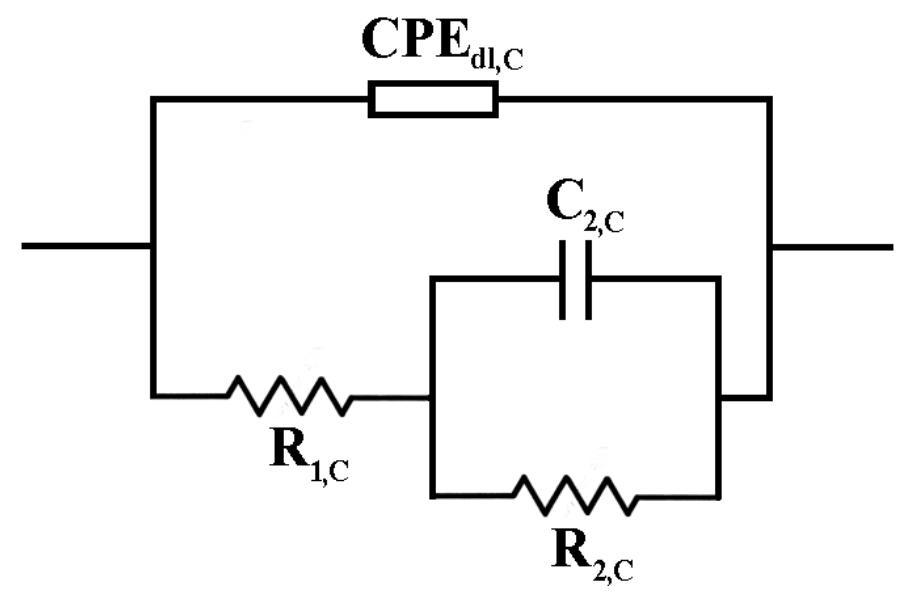

Figure 3.4 The PEM fuel cell cathode equivalent circuit

$$
C_{d l, C}=D_{C}=\frac{\varepsilon_{s}}{d+\frac{R T \varepsilon_{s}}{F \bar{\Gamma}_{\mathrm{H}^{+}}}}
$$




$$
\begin{aligned}
R_{1, C} & =\frac{1}{A_{C}}=\frac{R T}{\alpha_{C} n F \bar{j}} \\
R_{2, C} & =\frac{B_{C}}{A_{C} C_{C}}=\frac{l_{\mathrm{GDL}, \mathrm{C}} l_{\mathrm{CL}, \mathrm{C}}}{D_{\mathrm{GDL}, \mathrm{C}}} \frac{R T}{\alpha_{C} n^{2} F^{2} \bar{c}_{O, s}} \\
C_{2, C} & =\frac{A_{C}}{B_{C}}=\frac{\alpha_{C} n^{2} F^{2} \bar{c}_{O, s}}{R T}
\end{aligned}
$$

The double layer capacitance is replaced with a constant phase element to capture the electrode inhomogeneity effects

$$
Z_{\mathrm{CPE}, C}=\frac{1}{C_{d l, C}(i \omega)^{n_{\mathrm{CPE}, C}}}
$$

The values presented in Table 3.2 are used to study the equivalent circuit. The value of effective oxygen diffusivity in the cathode is considered to be smaller than the value of hydrogen diffusivity in the anode (see the values reported in $[2,110]$ ). The cathode double layer is considered to be $200 \mathrm{~F} \cdot \mathrm{m}^{-2}[117,172,173]$. The cathode transfer coefficient is assumed to be 0.47 (as reported in $[168,170,174,175])$. The adsorbed oxygen concentration $\left(\bar{c}_{O, s}\right)$ is assumed to be $5 \mathrm{e}-5 \mathrm{~mol} \cdot \mathrm{m}^{-2}$. This parameter has not been measured or reported in the literature. However, the discussions presented in Section 3.4 show that this assumed value is close to the values obtained from the best fit between the analytical and measured impedances.

Table 3.2 Parameters used for calculating the elements of the cathode equivalent circuit

\begin{tabular}{llcr} 
Property & Variable & Dimension & Value \\
\hline Cathode GDL thickness & $l_{\mathrm{GDL}, \mathrm{C}}$ & $\mathrm{m}$ & $4 \mathrm{e}-4$ \\
\hline Cathode CL thickness & $l_{\mathrm{CL}, \mathrm{C}}$ & $\mathrm{m}$ & $1 \mathrm{e}-5$ \\
\hline Cathode GDL diffusivity & $D_{\mathrm{GDL}, \mathrm{C}}$ & $\mathrm{m}^{2} \mathrm{~s}^{-1}$ & $1 \mathrm{e}-6$ \\
\hline Current density & $\bar{j}$ & $\mathrm{~A} \mathrm{~m}^{-2}$ & $0.3 \mathrm{e} 4$ \\
\hline Cathode double layer capacitance & $C_{d l, C}$ & $\mathrm{~F} \mathrm{~m}^{-2}$ & 200 \\
\hline Cathode transfer coefficient & $\alpha_{C}$ & - & 0.47 \\
\hline Adsorbed oxygen concentration & $\bar{c}_{O, s}$ & $\mathrm{~mol} \mathrm{~m}^{-2}$ & $5 \mathrm{e}-5$ \\
\hline Temperature & $T$ & ${ }^{\circ} \mathrm{C}$ & 60
\end{tabular}


Considering these values, it can be seen that the values of the resistances $R_{1, C}$ and $R_{2, C}$ are close, and hence, neither of them can be neglected (unlike the anode). As a result, the Nyquist plot from the equivalent circuit presented for the cathode consists of two convoluted semi-circles. Based on the values presented in Table 3.2, the arc has a diameter close to $0.23 \Omega \cdot \mathrm{cm}^{2}$ and the peak frequency of around $10 \mathrm{~Hz}$. The measured impedances in literature [20, 34, 46, 176] show there is an arc which has the same diameter and time constant. This arc is normally referred to as the mid-frequency (MF) arc [34]. It has to be mentioned that if the impedance of the capacitance $C_{2, C}$ becomes considerably lower than the impedance of the cathode double layer element, $Z_{\mathrm{CPE}, C}$, the cathode equivalent circuit shown in Figure 3.4 can result in two separate arcs. However, considering the values presented in Table 3.2 and also the cases studied in Section 3.4, this does not happen normally in PEM fuel cell impedances. Thus, the cathode impedance can be traced back to just one arc in the mid-frequency (MF) range, though this MF arc is often distorted as a result of the $C_{2, C}$ element.

It has been reported that as the current density increases, the diameter of the MF arc decreases to a minimum value and then slightly increases. This trend, which has also been observed in the measured impedances reported in the literature [20, 46, 114], can be explained using the equivalent circuit presented in Figure 3.4. As it can be seen from Equation (3.50), the $R_{1, C}$ resistance decreases as the current density increases. This causes the MF arc to shrink as the current density increases. However, as the current density increases, the rate of shrinkage decreases. Also, the adsorbed oxygen concentration starts to decrease which increases the $R_{2, C}$ resistance (Equation (3.51)). So, the resultant effect causes the MF arc to inflate in high current densities. This inflation of the MF arc in high current densities is in fact a sign of decreasing the adsorbed oxygen concentration in the cathode which is equivalent to cathode starvation. As a matter of fact, the cathode starvation can be captured from the MF arc for the current densities higher than the limit in which the MF arc inflates when the current density increases. It has to be mentioned that the low frequency (LF) arc also inflates as the current density increases, affecting the MF arc.

As mentioned above, variations in the absorbed oxygen concentration $\left(\bar{c}_{O, s}\right)$ affects the diameter and time constant of the MF arc. However, there is an upper limit for this effect (which is around $5 \mathrm{e}-3 \mathrm{~mol} \cdot \mathrm{m}^{-2}$ based on the values presented in Table 3.2). When the absorbed oxygen 
concentration increases more than this limit, it does not change the MF arc anymore. This limit can be considered as the "required oxygen limit" below which there is not "enough" reactant. Moreover, unlike anode starvation which cannot be captured in the HF arc in impedance measurements, the cathode starvation is clearly distinguishable from the variation in the MF arc (as discussed above).

\subsection{Validity of the Butler-Volmer equation estimations}

The Butler-Volmer equation can be written in the general form of [2]

$$
j=j_{0}\left(\frac{c_{R}}{c_{R}^{\text {Ref }}} \exp \left(\frac{\alpha n F \eta}{R T}\right)-\frac{c_{P}}{c_{P}^{\text {Ref }}} \exp \left(\frac{-(1-\alpha) n F \eta}{R T}\right)\right)
$$

where subscripts $R$ and $P$ and superscript Ref denote the reactants, products, and the reference values, respectively. Two different scenarios can be considered for the reference point which will be discussed in the following paragraphs.

The reference point can be considered as the point in which the net current equals zero. In other words,

First Scenario: $\quad c_{R}^{\mathrm{Ref}}=c_{R}(j=0), \quad c_{P}^{\mathrm{Ref}}=c_{P}(j=0)$

In this case, the overpotential $\eta$ denotes the potential difference between the reference point (the point in which the net current equals zero) and the working point. We call this overpotential, the Butler-Volmer overpotential $\left(\eta_{\mathrm{BV}}\right)$.

In the second scenario, the reference point can be defined as the point in which the current of the forward and reverse reactions are both zero. In this case,

Second Scenario: $\quad c_{R}^{\text {Ref }}=c_{R}(\vec{j}=0), \quad c_{P}^{\text {Ref }}=c_{P}(\overleftarrow{j}=0)$

In the above relations, the forward and reverse reactions are shown by ${ }^{\rightarrow}$ and ${ }^{-}$symbols, respectively. In this case, the overpotential not only consists of the potential loss due to the reaction $\left(\eta_{\mathrm{BV}}\right)$, but also the potential loss resulting from the Nernst equation (which is referred to as $\eta_{\mathrm{N}}$ ) [2]. In other words, in this scenario,

$\eta=\eta_{\mathrm{BV}}+\eta_{\mathrm{N}}$ 
The Nernst overpotential $\left(\eta_{\mathrm{N}}\right)$ is the result of the change in the reversible voltage $\left(E^{0}\right)$ due to the reactant and product activities. In fact, the Nernst overpotential depicts the potential loss (at equilibrium state $(j=0))$ as a result of a decrease in the reactant concentration or an increase in the product concentration.

In the cathode side, the second scenario has been considered for the Butler-Volmer equation (Equation (3.39)), and hence, the Nernst loss has been considered in the overpotential parameter $\left(\eta_{C}\right)$, even though the Nernst loss can be expected to be negligible as the cathode exchange current density is in the order of $1 \mathrm{e}-9 \mathrm{~A} \cdot \mathrm{cm}^{-2}[168-172]$.

In the anode side, however, the first scenario has been used for the Butler-Volmer equation (Equation (3.16)). As a result, the anode overpotential shown in the Butler-Volmer equation $\left(\eta_{A}\right)$ only consists of the reaction loss. In other words,

$$
\eta_{A}=\eta_{A, \mathrm{BV}}
$$

This overpotential is the overpotential which has been reported to be negligible in literature [34, 141] and has been linearized in Equation (3.18). In this case, the Nernst loss has been hidden in the concentration terms. Thus, the reference concentrations of $c_{\mathrm{H}_{2}}^{0}$ (i.e., the reference reactant concentration) and $c_{\mathrm{H}^{+}, \mathrm{s}}^{0}$ (i.e., the reference product concentration) have different orders of magnitude (see the next paragraph in this section). However, the ratios of $\frac{c_{\mathrm{H}_{2}}}{c_{\mathrm{H}_{2}}^{0}}$ and $\frac{c_{\mathrm{H}^{+}, s}}{c_{\mathrm{H}^{+}, \mathrm{s}}^{0}}$ are close to unity, as the concentration changes (resulting from the net reaction) are negligible. Consequently, the $\left(\frac{c_{\mathrm{H}_{2}}}{c_{\mathrm{H}_{2}}^{0}}-\left(\frac{c_{\mathrm{H}^{+}, s}}{c_{\mathrm{H}^{+}, \mathrm{s}}^{0}}\right)^{2}\right)$ term can be ignored. Clearly, if the anode Butler-Volmer equation is considered based on the second scenario, the overpotential cannot be linearized and the ratios of $\frac{c_{\mathrm{H}_{2}}}{c_{\mathrm{H}_{2}}^{0}}$ and $\frac{c_{\mathrm{H}^{+}, s}}{c_{\mathrm{H}^{+}, \mathrm{s}}^{0}}$ have different orders of magnitude. Obviously, the $\left(\frac{c_{\mathrm{H}_{2}}}{c_{\mathrm{H}_{2}}^{0}}-\left(\frac{c_{\mathrm{H}^{+}, s}}{c_{\mathrm{H}^{+}, \mathrm{s}}^{0}}\right)^{2}\right)$ term is not negligible in this case.

Even though the first scenario is used for the anode reaction, the Nernst loss is still important and is reflected in the difference between the orders of magnitude of the reference concentrations (mentioned above). To show how the order of the magnitudes of these concentrations are 
different, the Butler-Volmer equation for the anode at the equilibrium state can be written based on the activation barriers [2] using the second scenario.

$$
j_{0, A}=\overrightarrow{j_{A}}=\overleftarrow{j_{A}}=n F \frac{c_{\mathrm{H}_{2}}^{j=0}}{c_{\mathrm{H}_{2}}^{0}} \vec{h} \exp \left(\frac{-\overrightarrow{\Delta G}}{R T}\right)=n F\left(\frac{c_{\mathrm{H}^{+}, \mathrm{s}}^{j=0}}{c_{\mathrm{H}^{+}, \mathrm{s}}^{0}}\right)^{2} \overleftarrow{h} \exp \left(\frac{-\left(\overleftarrow{\Delta G}-\Delta G_{r x n}\right)}{R T}\right)
$$

In the above equation, $h$ and $\Delta G$ denote the decay rate and the activation barrier, respectively. As the exchange current density of this reaction is considerably large [138, 145-154], the rate of the reaction in the equilibrium will be high. Considering the values reported for the activation barrier of the forward reaction $\left(\overrightarrow{\Delta G} \approx 29 \mathrm{~kJ} \cdot \mathrm{mol}^{-1}\right.$ [138]) and the net activation barrier $\left(\Delta G_{r x n} \approx-44 \mathrm{~kJ} \cdot \mathrm{mol}^{-1}[177]\right)$, one can conclude that the concentration of the product (proton) is high. This excess concentration is the reason for the considerable Nernst overpotential which mainly constructs the anode loss and is captured in the HF arc.

\subsection{Comparison with measured impedances}

The impedances calculated from the equivalent circuits presented for the anode and cathode are compared against the measured impedances reported in literature [20, 34, 46]. Two sets of experiments (one reported by Malevich et al. [46] and the other measured by Danzer and Hofer [20]) selected here for verification of the model are examples of different categories of the measured impedances reported in literature. Figure 3.5.a compares the measured impedances presented by Malevich et al. [46] and the impedances of the presented analytical solution. To conduct this comparison, the lowest current density $\bar{j}=0.21 \mathrm{~A} \cdot \mathrm{cm}^{-2}$ is first fitted to the

analytical solution to obtain the values of $j_{0, A}=1200 \mathrm{~A} \cdot \mathrm{m}^{-2}, n_{\mathrm{CPE}, \mathrm{A}}=n_{\mathrm{CPE}, \mathrm{C}}=0.87, \alpha_{C}=0.4$, $C_{d l, C}=350 \mathrm{~F} \cdot \mathrm{m}^{-2}, \bar{c}_{O, s}=1.08 \mathrm{e}-4 \mathrm{~mol} \cdot \mathrm{m}^{-2}$ and $D_{\mathrm{GDL}, \mathrm{C}}=2.2 \mathrm{e}-6 \mathrm{~m}^{2} \cdot \mathrm{s}^{-1}$. It is noted that the cathode transfer coefficient $\left(\alpha_{C}=0.4\right)$ is smaller than the measured ORR transfer coefficients (0.47) $[168,170,174,175]$. However, it was shown that the physical and morphological properties of the electrode can increase the Tafel slope and hence decrease the transfer coefficient considerably [178]. The anode and cathode CPE exponents are assumed to be equal as it is expected to be related to the electrodes physical properties (as discussed in Chapter 1). Using these values obtained from fitting the analytical solution to the measured impedances at the lowest current density, the Nyquist plots in other current densities are determined from the presented solution and compared against the measured impedances. In each case, the above- 
mentioned values are kept constant while the current density is changed based on the values presented in [46]. As it can be seen, there is excellent agreement in the HF arc which shows the anode impedance.
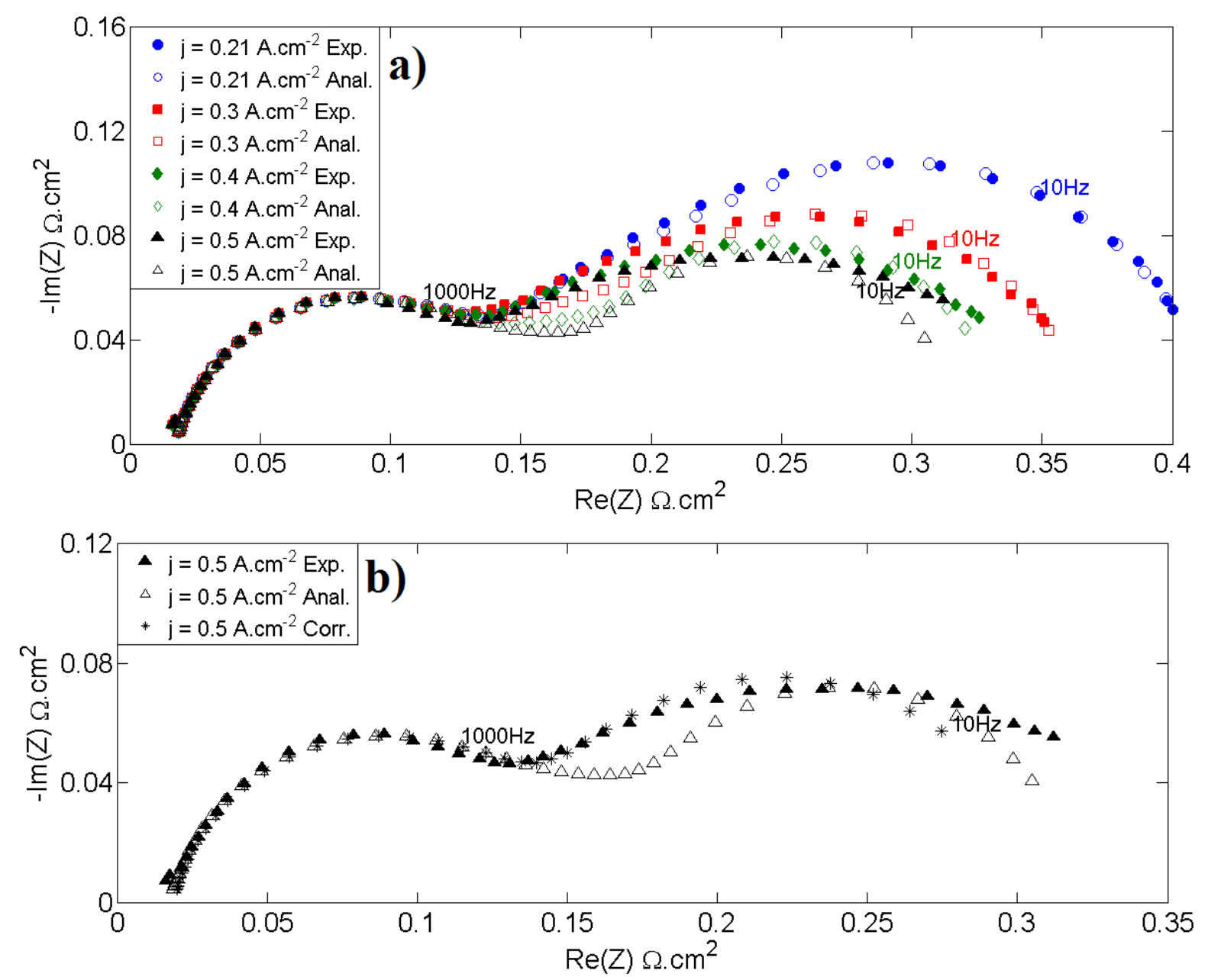

Figure 3.5 (a) Comparison of the measured impedances presented by Malevich et al. [46] and the predicted impedances from the analytical solution; (b) The effect of the oxygen concentration correction is shown by asterisks

In the MF arc (corresponding to the cathode impedance), although the arc diameter and time constant obtained through the analytical solution are in good agreement with the measured values, there is a discrepancy at the high current densities around the frequency of $100 \mathrm{~Hz}$ where the real part of the impedance is around $0.15 \Omega \cdot \mathrm{cm}^{2}$ (with the maximum discrepancy of $16 \%$ ). However, it has to be remembered that the absorbed oxygen concentration $\left(\bar{c}_{O, s}\right)$ is assumed to remain constant; while due to cathode starvation, this concentration is reduced when the current 
density increases. If this concentration reduction is considered and the absorbed oxygen concentration $\left(\bar{c}_{O, s}\right)$ is adjusted accordingly, the values of $R_{2, C}$ and $C_{2, C}$ elements are increased and decreased, respectively, which results in better agreement between the theoretical and measured impedances. As an example, this improvement is shown in Figure 3.5.b for the highest current density (with the discrepancy less than 3\%). It has to be mentioned that the HF and MF arcs are just considered here for verification; whereas the low frequency (LF) arcs of these measured impedances were used in Chapter 2 for the verification of the ohmic-loss analysis. Moreover, the impedances measured and reported by Malevich et al. [46] at current densities higher than $0.5 \mathrm{~A} \cdot \mathrm{cm}^{-2}$ are not considered for model verification here since at these high current densities the concentration of the absorbed oxygen cannot be assumed constant.

The same method is employed and the results are compared with the measured impedances presented by Danzer and Hofer [20] (see Figure 3.6.a). The lowest current density is used to fit the solution to the measured impedances. The fit has resulted in obtaining the values of $j_{0, A}=3000 \mathrm{~A} \cdot \mathrm{m}^{-2}, n_{\mathrm{CPE}, \mathrm{A}}=n_{\mathrm{CPE}, \mathrm{C}}=0.95, \alpha_{C}=0.35, C_{d l, C}=40 \mathrm{~F} \cdot \mathrm{m}^{-2}, \bar{c}_{O, s}=9 \mathrm{e}-6 \mathrm{~mol} \cdot \mathrm{m}^{-2}$ and $D_{\mathrm{GDL}, \mathrm{C}}=2 \mathrm{e}-5 \mathrm{~m}^{2} \cdot \mathrm{s}^{-1}$. It is clear that the reported measured impedances included in Figure 3.5.a are considerably different from those included in Figure 3.6.a (in which the HF arc is more convoluted in the MF arc) since they are obtained by different groups using different fuel cell setups running at different operating conditions. Despite this difference in the two sources of measurements, the presented equivalent circuit can still capture the trend. Similar to the previous figure, the values obtained from the fit at the lowest current density are then used to predict the Nyquist plots in other measured current densities. In general, there is good agreement between the measured impedances and those obtained through the analytical model presented here. Again, the discrepancy between the theoretical and experimental values can be reduced by modifying the absorbed oxygen concentration which is shown by asterisk symbols in Figure 3.6.b for the highest current density (reducing the maximum discrepancy between the experimental and model results from $20 \%$ to $2 \%$ ). Here again, the LF arcs are not shown as those have been used to verify the membrane model presented before in Chapter 2. Moreover, the high current densities are again excluded from the verification studies as the absorbed oxygen concentration cannot be assumed constant at high current densities. 

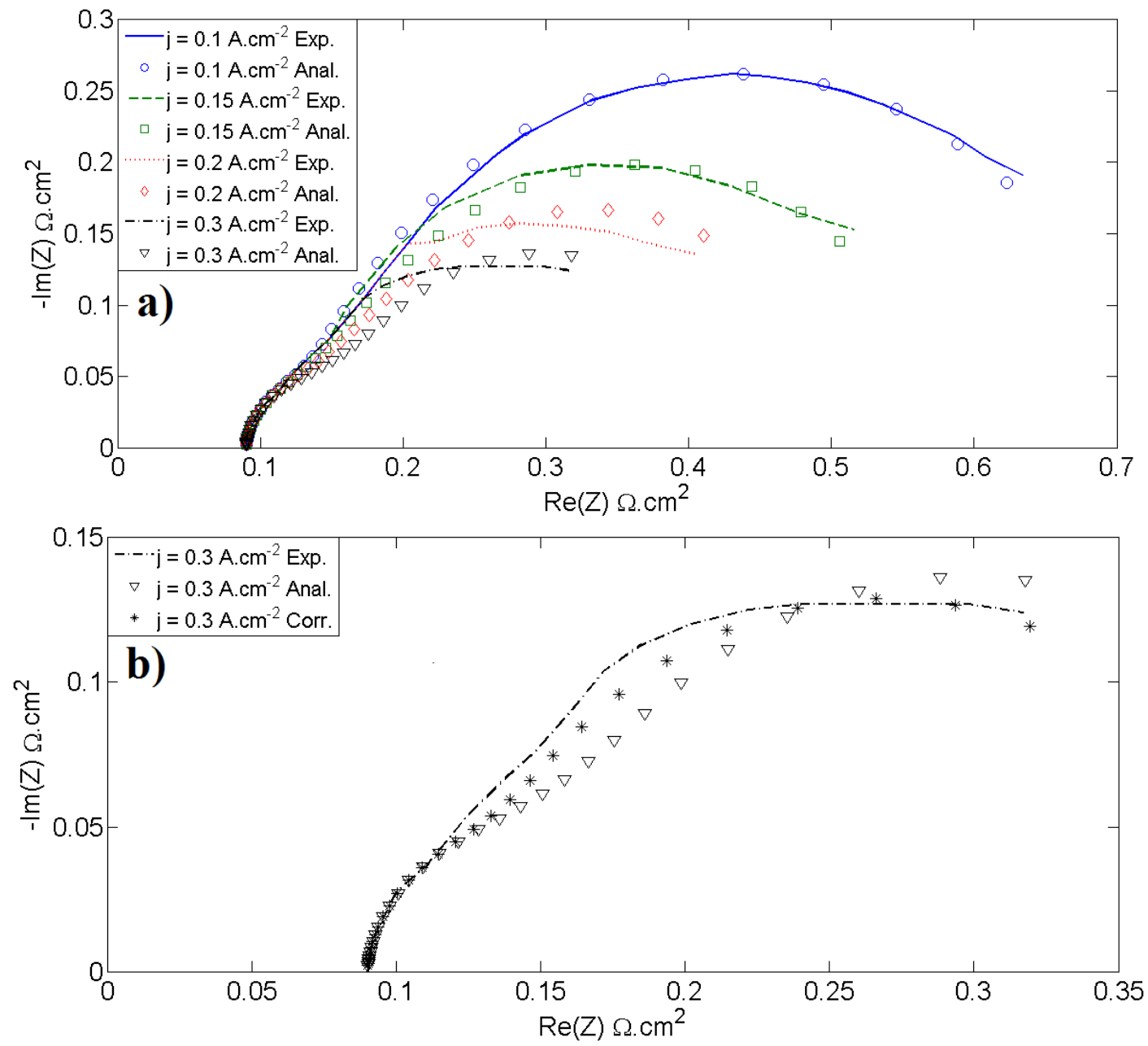

Figure 3.6 (a) Comparison of the measured impedances presented by Danzer and Hofer [20] and the predicted impedances from the analytical solution; (b) The effect of the oxygen concentration correction is shown by asterisks

Finally, Figure 3.7 compares the theoretical and measured impedances for the cases where different streams have been fed through the anode and cathode. The analytical model has been fitted to the measured impedances reported for the $\mathrm{H}_{2}$ /Air feeding case. As a result, the values of $C_{d l, A}=30 \quad \mathrm{~F} \cdot \mathrm{m}^{-2}, \quad j_{0, A}=1600 \quad \mathrm{~A} \cdot \mathrm{m}^{-2}, \quad n_{\mathrm{CPE}, \mathrm{A}}=n_{\mathrm{CPE}, \mathrm{C}}=0.9, \quad \alpha_{C}=0.4, \quad C_{d l, C}=500 \quad \mathrm{~F} \cdot \mathrm{m}^{-2}$, $\bar{c}_{O, s}=7.2 \mathrm{e}-5 \mathrm{~mol} \cdot \mathrm{m}^{-2}$ and $D_{\mathrm{GDL}, \mathrm{C}}=2.2 \mathrm{e}-6 \mathrm{~m}^{2} \cdot \mathrm{s}^{-1}$ are obtained. The measured impedance has a low frequency (LF) arc presenting the ohmic loss in the membrane (as discussed in Chapter 2) and is not considered here. For the $\mathrm{H}_{2} / \mathrm{O}_{2}$ feeding case, all the parameters are kept constant while the cathode GDL diffusion coefficient is modified to $D_{\mathrm{GDL}, \mathrm{C}}=7.8 \mathrm{e}-6 \mathrm{~m}^{2} \cdot \mathrm{s}^{-1}$. As it can be seen, the analytical solution can capture both the arcs' diameters and time constants of the measured 
impedances well (with the maximum discrepancy of 6\%). Next, the same parameters are employed for the $\mathrm{H}_{2} / \mathrm{H}_{2}$ feeding case for which the cathode equivalent circuit is eliminated. The agreement is also excellent in this case. It has to be mentioned that the cathode GDL diffusion coefficient used in the model cannot be considered as an accurate estimation for the cathode diffusivity as the effect of the nitrogen gas in the air on the diffusion process has not been counted in the modeling process.

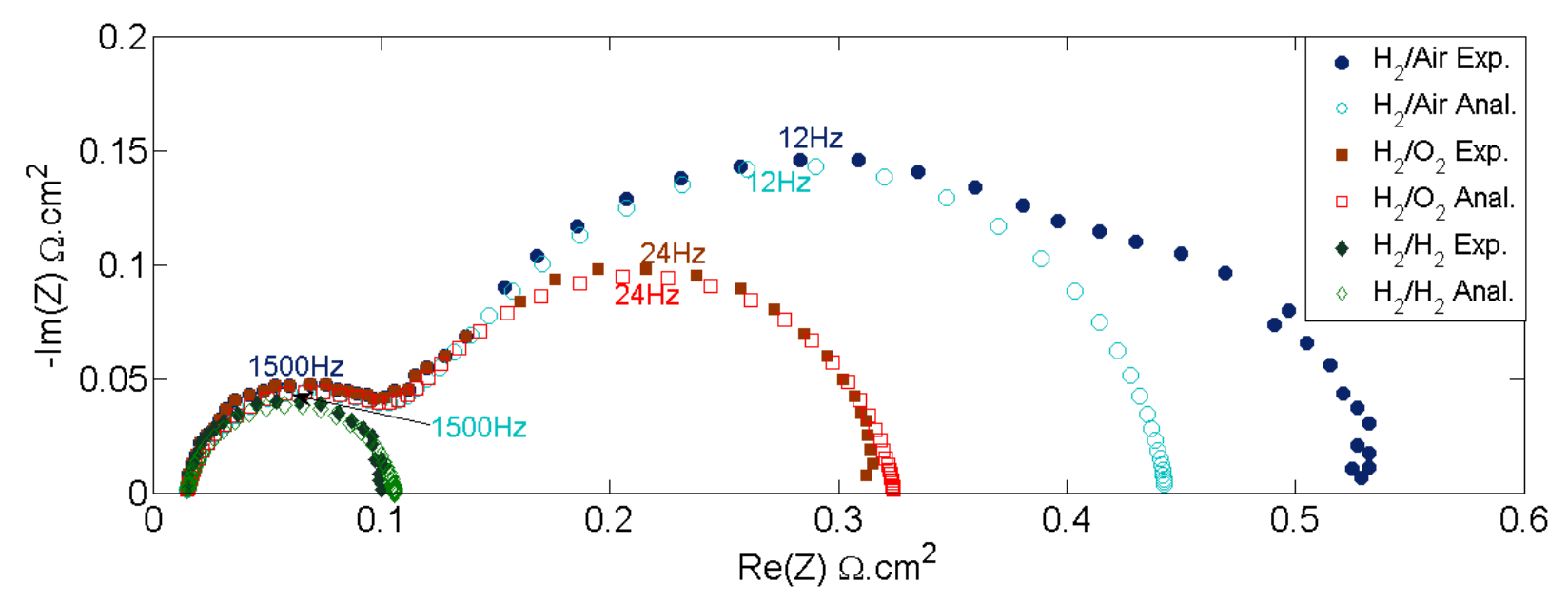

Figure 3.7 Comparison of the measured impedances presented by Malevich et al. [34] and the predicted impedances from the analytical solution

\subsection{Concluding remarks}

The anode and cathode electrodes of the proton exchange membrane fuel cell were studied analytically and the resultant equivalent circuits were determined. The results were compared against different measured impedances reported for various current densities and anode/cathode feedings. It was shown that the presented analytical solution is in great agreement with the measured impedances. The high frequency (HF) arc was determined to be related to the anode electrode impedance and could be modeled via a parallel combination of the anode double layer constant phase element and a resistance which is analytically calculated. The mid-frequency (MF) arc was shown to be related to the cathode impedance. The presented equivalent circuits showed that the HF arc is not a function of the current density while the MF arc shrinks and then inflates as the current density increases which is in agreement with the reported trends in the literature. It was also shown that cathode starvation can be captured from the MF arc. 


\section{Chapter 4. Verification of the model}

In this chapter, the complete process model for the PEM fuel cell is presented. The impedance of the ohmic, activation, and mass transport overpotentials analyzed in Chapters 2 and 3 are reviewed first. Then, the impedances are combined and the complete process model for PEM fuel cells is presented. The model is verified against measured impedances obtained at different current densities, operating temperatures, and anode and cathode relative humidities. It is shown that the model can predict the measured impedances in all different operating conditions extremely well. The measured impedances of the PEM fuel cells reported in literature are then categorized and the physicochemical interpretations of different parts of the Nyquist plots are explained and clarified.

\subsection{Theory}

As discussed in previous chapters, the impedance of the cell needs to be analytically determined in a process model by calculating the ratio of the perturbed part of the potential to the perturbed part of the current. The potential is considered as a function of the activation, ohmic and mass transport overpotentials which are the most dominant losses in the PEM fuel cell [2]. In other words,

$V=E_{\text {theor. }}-\eta_{\text {act. }}-\eta_{\text {ohm. }}-\eta_{\text {trans. }}$

As a result, the impedance of the cell can be calculated as the summation of the impedances of these losses.

$Z_{\text {tot }}=Z_{\text {act. }}+Z_{\text {trans. }}+Z_{\text {ohm }}$

These impedances are shortly reviewed separately in the following sections and the overall equivalent circuit and the analytical relations of the elements of the circuit are then presented. 


\subsubsection{Activation and mass transport overpotentials}

To determine the impedance of these overpotentials, the anode and cathode electrodes were studied separately in Chapter 3. It has to be mentioned that the complete version of the ButlerVolmer equation which also includes the concentrations of the reactants and products, can model both the activation and mass transport losses, simultaneously [2]. Considering the insignificant overpotential of the anode in the reaction compared to that of the cathode [179-181], the ButlerVolmer equation can be linearized for the anode electrode (even at the high current densities) with acceptable accuracy.

$$
j_{\mathrm{H}^{+}}=j_{0, A}\left(\frac{c_{\mathrm{H}^{+}, \mathrm{s}}}{c_{\mathrm{H}^{+}, \mathrm{s}}^{0}}\right)^{2} \frac{n F \eta_{A}}{R T}
$$

For the cathode, however, as the exchange current density is extremely small [e.g., 168, 170], it was shown in Chapter 3 that the Tafel assumption is extremely accurate in all working current densities.

$$
j_{\mathrm{C}}=j_{0, C} \frac{c_{\mathrm{O}, \mathrm{s}}}{c_{\mathrm{O}, \mathrm{s}}^{0}} \exp \left(\frac{\alpha_{C} n F \eta_{C}}{R T}\right)
$$

The thorough discussion about the validity of these assumptions and the consistency of the resulted conclusions with the measured impedances were presented in Chapter 3. To determine the impedances of the anode and the cathode electrodes, the adsorbed proton (or the oxygen atom) concentrations have to be determined. It was shown that

$$
\frac{\partial c_{\mathrm{H}^{+}, \mathrm{s}}}{\partial t}=-\frac{j_{\mathrm{H}^{+}}}{F}+2 D_{\mathrm{GDL}, \mathrm{A}} \frac{\partial c_{\mathrm{H}_{2}, \mathrm{~s}}}{\partial x}
$$

The above equation estimates the temporal change of the proton concentration from the consumption (the first term on the right hand side) and the diffusion (the second term on the right hand side) terms. A Similar equation can be considered for the oxygen atom concentration. Finally, the impedance of the anode and cathode can be determined by perturbing the above equations which are presented below. 


$$
\begin{gathered}
\frac{1}{Z_{A}}=\frac{j_{0, A}\left(\frac{\bar{c}_{\mathrm{H}^{+}, \mathrm{s}}}{c_{\mathrm{H}^{+}, \mathrm{s}}^{0}}\right)^{2} \frac{n F}{R T}}{1+j_{0, A} \frac{2 \bar{c}_{\mathrm{H}^{+}, \mathrm{s}}}{\frac{n F \bar{\eta}_{A} /(R T)}{c_{\mathrm{H}^{+}, \mathrm{s}}^{0}} F\left(i \omega+\frac{D_{\mathrm{GDL}, \mathrm{A}}}{l_{\mathrm{GDL}, \mathrm{A}} l_{\mathrm{CL}, \mathrm{A}}}\right)}}+\varepsilon_{s}(i \omega) \frac{1}{d+\frac{R T \varepsilon_{s}}{F \bar{\Gamma}_{\mathrm{H}^{+}}}} \\
\frac{1}{Z_{C}}=\frac{j_{0, C} \frac{\bar{c}_{\mathrm{O}, \mathrm{s}}}{c_{\mathrm{O}, \mathrm{s}}^{0}} \exp \left(\frac{\alpha_{C} n F \bar{\eta}_{C}}{R T}\right) \frac{\alpha_{C} n F}{R T}}{1+\frac{j_{0, C}}{c_{\mathrm{O}, \mathrm{s}}^{0}} \frac{\exp \left(\frac{\alpha_{C} n F \bar{\eta}_{C}}{R T}\right)}{n F\left(i \omega+\frac{R D_{\mathrm{GDL}, \mathrm{C}}}{l_{\mathrm{GDL}, \mathrm{C}} l_{\mathrm{CL}, \mathrm{C}}}\right)}}+\varepsilon_{s}(i \omega) \frac{1}{d+\frac{R T \varepsilon_{s}}{F \bar{\Gamma}_{\mathrm{H}^{+}}}}
\end{gathered}
$$

The details of the calculations and deriving steps were covered in Chapter 3 where the predicted impedances were compared against measured impedances of different PEM fuel cells reported in literature. The impedance relations were also converted to equivalent circuits which are shown in Figures 4.1.a and 4.1.b. It has to be clarified that Equation (4.6) generally results in an equivalent circuit similar to that of Equation (4.7); however, as discussed in Chapter 3, two elements in the equivalent circuit resulted from Equation (4.6) are orders of magnitude lower than other elements and can be safely ignored (see Figures 4.1.a and 4.1.b).
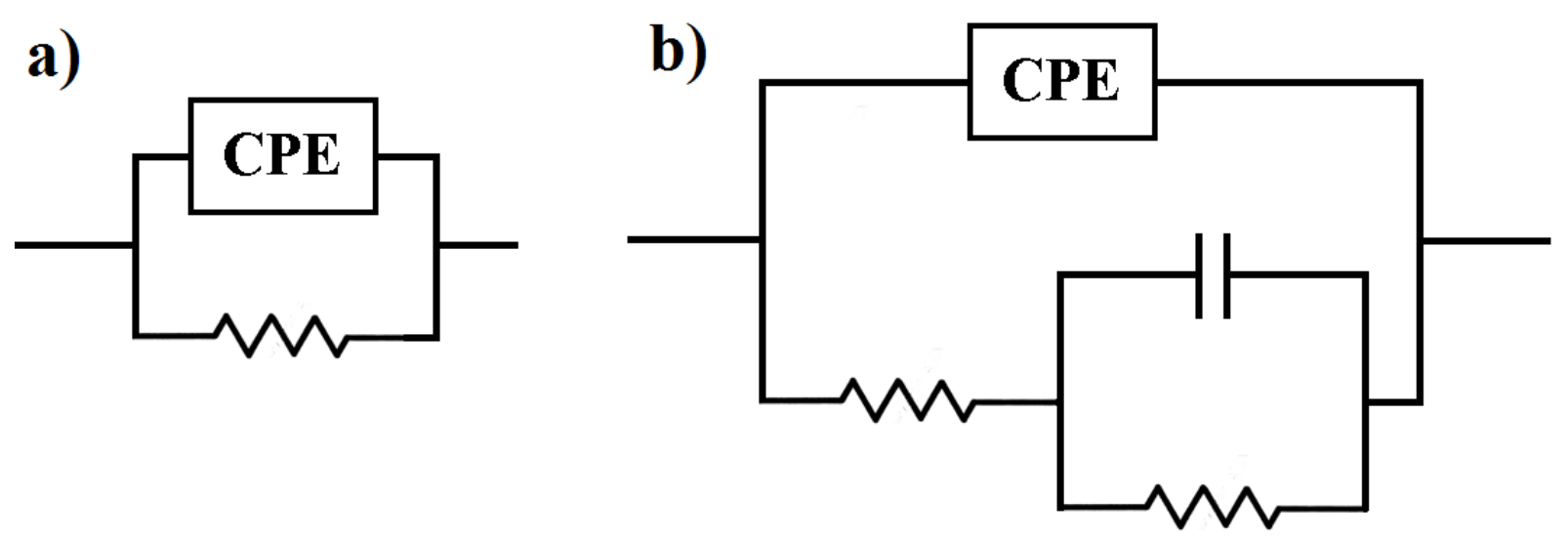

Figure 4.1 The equivalent circuits for (a) anode (resulted from Equation (4.6)), and (b) cathode (Equation (4.7)) 


\subsubsection{Ohmic overpotential}

The ohmic overpotential can be determined by formulating the membrane conductivity as a function of the membrane water content (as discussed in Chapter 2). The membrane water content can be estimated by considering the water flux by the proton transfer and back diffusion phenomena in the membrane [2]

$$
\frac{\rho_{\mathrm{M}}}{M_{\mathrm{M}}} \frac{\partial \lambda_{\mathrm{M}}}{\partial t}=-\alpha j \frac{\partial \lambda_{\mathrm{M}}}{\partial x}+\beta \frac{\partial^{2} \lambda_{\mathrm{M}}}{\partial x^{2}}
$$

To estimate the space derivations in the above relation, the water concentration in the cathode catalyst layer needs to be obtained. This concentration can be obtained by estimating the water production as a result of the chemical reactions, the water flux from the catalyst layer towards the gas diffusion layer, and the water flux from the membrane towards the cathode catalyst layer.

$$
\frac{\partial c_{\mathrm{CL}}^{\mathrm{w}}}{\partial t}=\frac{j}{2 F l_{\mathrm{CL}}}-\frac{J_{\mathrm{GDL}}}{l_{\mathrm{CL}}}+\frac{J_{\mathrm{M}}}{l_{\mathrm{CL}}}
$$

By perturbing Equations (4.8) and (4.9), the impedance of the ohmic loss can be finally determined as

$$
Z_{\text {ohm. }}=\frac{l_{\mathrm{M}}}{\bar{\sigma}_{\mathrm{M}}}-\frac{l_{\mathrm{M}} a \bar{j}}{\bar{\sigma}_{\mathrm{M}}^{2}} \frac{-\alpha \bar{\lambda}_{\mathrm{M}}+\frac{2 \beta}{l_{\mathrm{M}}} \frac{\frac{M_{\mathrm{M}}}{2 F\left(l_{\mathrm{CL}}+f l_{\mathrm{GDL}}\right) \rho_{\mathrm{M}}}}{i \omega+\frac{D_{\mathrm{GDL}}^{\mathrm{w}} \eta_{c h}}{\left(l_{\mathrm{CL}}+f l_{\mathrm{GDL}}\right) l_{\mathrm{GDL}}}}}{\frac{l_{\mathrm{M}} \rho_{\mathrm{M}}}{M_{\mathrm{M}}} i \omega+\left(\alpha \bar{j}+\frac{2 \beta}{l_{\mathrm{M}}}\right)-\frac{2 \beta}{l_{\mathrm{M}}} \frac{\frac{l_{\mathrm{M}}}{l_{\mathrm{CL}}+f l_{\mathrm{GDL}}} i \omega+\frac{D_{\mathrm{GDL}}^{\mathrm{w}} \eta_{c h}}{\left(l_{\mathrm{CL}}+f l_{\mathrm{GDL}}\right) l_{\mathrm{GDL}}}}{i \omega}}
$$

The details of the derivation process of the above equation have been explained in Chapter 2. It was also shown that this impedance relation can be converted to an equivalent circuit presented in Figure 4.2 (with the impedance relation shown in Equation (4.11)). The impedances predicted by this analysis were compared against measured impedances, and it was shown that the analytical results are in great agreement with the measured impedances reported in literature.

$$
Z_{\text {circuit }}=R_{0}+\frac{R_{1} R_{2}+R_{1} L(i \omega)}{\left(R_{1}+R_{2}\right)+\left(R_{1} R_{2} C+L\right)(i \omega)+R_{1} L C(i \omega)^{2}}
$$




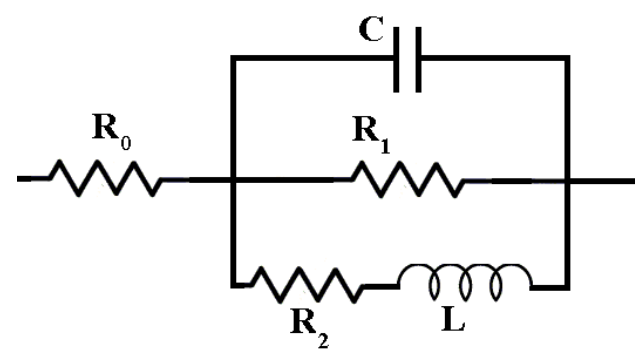

Figure 4.2 The equivalent circuit for the ohmic loss (Equation (4.10))

\subsubsection{Overall impedance}

Considering the impedance relations and equivalent circuits presented in the above sections, the overall impedance of the PEM fuel cell can be determined as

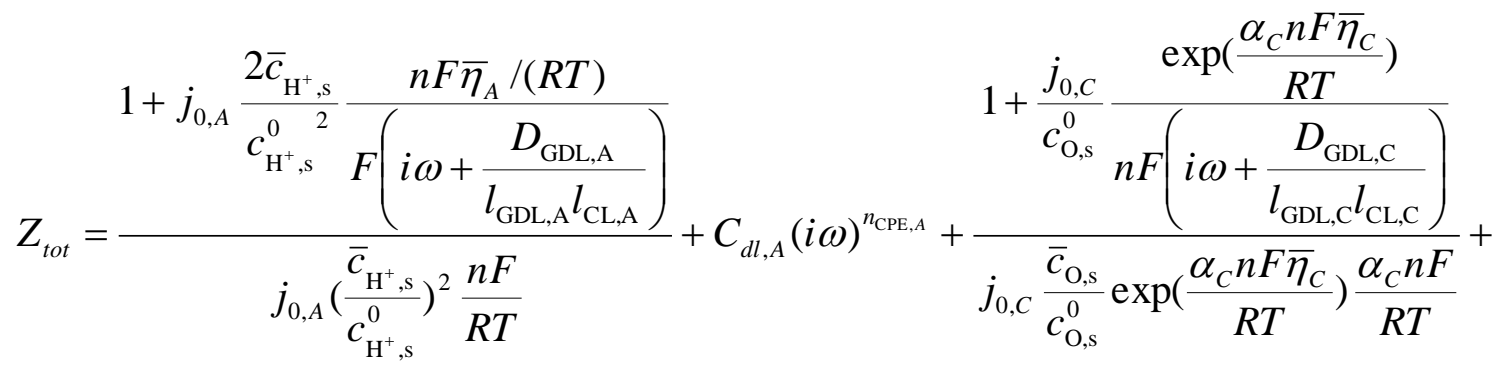

$$
\begin{aligned}
& -\alpha \bar{\lambda}_{\mathrm{M}}+\frac{2 \beta}{l_{\mathrm{M}}} \frac{\frac{M_{\mathrm{M}}}{2 F\left(l_{\mathrm{CL}}+f l_{\mathrm{GDL}}\right) \rho_{\mathrm{M}}}}{i \omega+\frac{D_{\mathrm{GDL}}^{\mathrm{w}} \eta_{c h}}{\left(l_{\mathrm{CL}}+f l_{\mathrm{GDL}}\right) l_{\mathrm{GDL}}}} \\
& \frac{l_{\mathrm{M}} \rho_{\mathrm{M}}}{M_{\mathrm{M}}} i \omega+\left(\alpha \bar{j}+\frac{2 \beta}{l_{\mathrm{M}}}\right)-\frac{2 \beta}{l_{\mathrm{M}}} \frac{\frac{l_{\mathrm{M}}}{l_{\mathrm{CL}}+f l_{\mathrm{GDL}}} i \omega+\frac{D_{\mathrm{GDL}}^{\mathrm{w}} \eta_{c h}}{\left(l_{\mathrm{CL}}+f l_{\mathrm{GDL}}\right) l_{\mathrm{GDL}}}}{i \omega}
\end{aligned}
$$

Similarly, the equivalent circuit of the PEM fuel cell can be determined by combining the abovementioned equivalent circuits. Figure 4.3 shows this overall equivalent circuit. As discussed in Chapters 2 and 3, the elements of this equivalent circuit can be calculated from the impedance relations as 


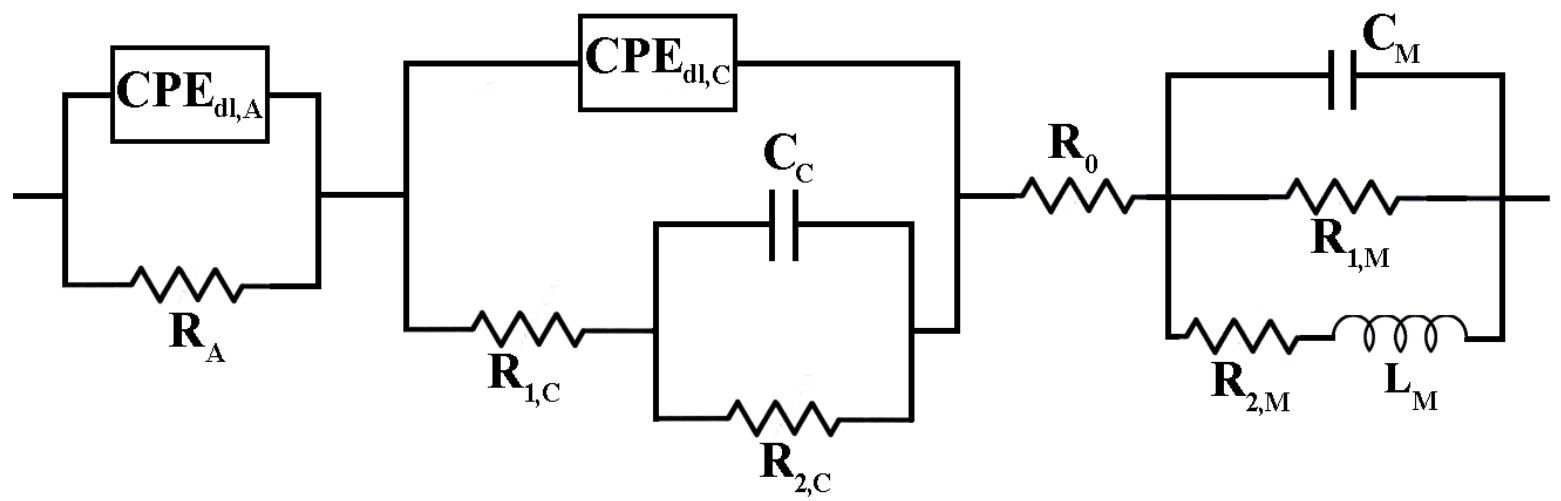

Figure 4.3 The overall equivalent circuit of the PEM fuel cell

$$
\begin{aligned}
& R_{A}=\frac{1}{j_{0, A}} \frac{R T}{n F} \\
& R_{1, C}=\frac{R T}{\alpha_{C} n F \bar{j}} \\
& C_{C}=\frac{\alpha_{C} n^{2} F^{2} \bar{c}_{\mathrm{O}, \mathrm{s}}}{R T} \\
& R_{2, C}=\frac{l_{\mathrm{GDL}, C} l_{\mathrm{CL}, C}}{D_{\mathrm{GDL}, C}} \frac{R T}{\alpha_{C} n^{2} F^{2} \bar{c}_{\mathrm{O}, \mathrm{s}}} \\
& R_{0}=\frac{l_{\mathrm{M}}}{\bar{\sigma}_{\mathrm{M}}} \\
& C_{\mathrm{M}}=\frac{\bar{\sigma}_{\mathrm{M}}^{2}}{l_{\mathrm{M}} a \bar{j}} \frac{l_{\mathrm{M}} \rho_{\mathrm{M}}}{\alpha \bar{\lambda}_{\mathrm{M}} M_{\mathrm{M}}}
\end{aligned}
$$

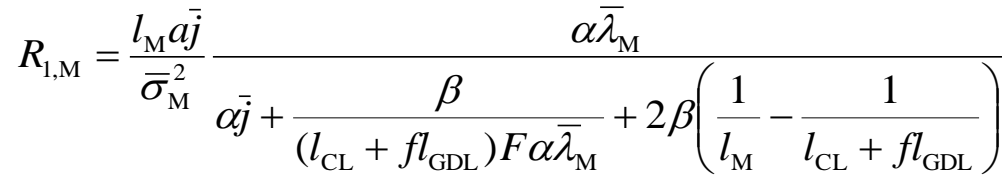

$$
\begin{aligned}
& R_{2, \mathrm{M}}=\frac{l_{\mathrm{M}} a \bar{j}}{\bar{\sigma}_{\mathrm{M}}^{2}} \frac{\alpha \bar{\lambda}_{\mathrm{M}}}{\frac{2 \beta}{l_{\mathrm{CL}}+f l_{\mathrm{GDL}}}\left(1-\frac{1}{2 F \alpha \bar{\lambda}_{\mathrm{M}}}\right)-\frac{\alpha \bar{j}+\frac{2 \beta}{l_{\mathrm{M}}}}{1-\frac{D_{\mathrm{GDL}}^{\mathrm{w}} \eta_{c h} F \rho_{\mathrm{M}} l_{\mathrm{M}} \alpha \bar{\lambda}_{\mathrm{M}}}{l_{\mathrm{GDL}} \beta M_{\mathrm{M}}}}}
\end{aligned}
$$




$$
L_{\mathrm{M}}=\frac{l_{\mathrm{M}} a \bar{j}}{\bar{\sigma}_{\mathrm{M}}^{2}} \frac{\frac{\alpha^{2} \bar{\lambda}_{\mathrm{M}}^{2} l_{\mathrm{M}} F\left(l_{\mathrm{CL}}+f l_{\mathrm{GDL}}\right) \rho_{\mathrm{M}}}{\beta M_{\mathrm{M}}}}{\left(\alpha \bar{j}+\frac{2 \beta}{l_{\mathrm{M}}}\right)-\frac{2 \beta}{l_{\mathrm{CL}}+f l_{\mathrm{GDL}}}\left(1-\frac{1}{2 F \alpha \bar{\lambda}_{\mathrm{M}}}\right)\left(1-\frac{D_{\mathrm{GDL}}^{\mathrm{w}} \eta_{c h} F \rho_{\mathrm{M}} l_{\mathrm{M}} \alpha \bar{\lambda}_{\mathrm{M}}}{l_{\mathrm{GDL}} \beta M_{\mathrm{M}}}\right)}
$$

\subsection{Experimental setup}

A $7.98-\mathrm{cm}^{2}$ PEM fuel cell was used for this thesis. The cell has 9 parallel channels with the length of $38 \mathrm{~mm}$ each in both anode and cathode sides (each channel is $1.5 \mathrm{~mm}$ wide and $0.5 \mathrm{~mm}$ deep and the land is $0.938 \mathrm{~mm}$ wide). Two coolant channels were assembled on both sides of the cell to control the temperature of the cell by the coolant (water) temperature. Two SGL-25BC (SIGRACET Fuel Cell Components) gas diffusion layers (with the thickness of 235 microns and PTFE loading of 10\%) for the anode and cathode sides with an XL-3BP-318 membrane electrode assembly (Yangtze Energy Technologies) (with the membrane thickness of 27.5 microns and the catalyst layer thickness of 12 microns each) sandwiched between them were used. The cell was controlled and monitored using the Arbin fuel cell test station.

The impedance measurements were accomplished using VERSASTAT 4 electrochemical system (Princeton Applied Research). The impedance was measured in each operating condition in the frequency range of $10 \mathrm{kHz}-10 \mathrm{mHz}$ at 10 points per decade rate. All the impedance measurements were performed in the potentiostatic mode, while the AC perturbation voltage was set to $25 \mathrm{mV}$ which is smaller than the thermal voltage $[1,36]$. The impedance of the cell was measured at anode and cathode flow rates of 0.55 SLPM (standard litres per minute) and 1.42 SLPM, respectively (stoichiometry ratio of 5 at $2 \mathrm{~A} \cdot \mathrm{cm}^{-2}$ ), operating (cell and reactants) temperatures of $65{ }^{\circ} \mathrm{C}, 70{ }^{\circ} \mathrm{C}$ and $75{ }^{\circ} \mathrm{C}$, anode and cathode relative humidities of $80 \%, 90 \%$ and $100 \%$, and potentials of $0.8 \mathrm{~V}, 0.75 \mathrm{~V}, 0.7 \mathrm{~V}, 0.65 \mathrm{~V}$ and $0.6 \mathrm{~V}$.

\subsection{Results}

To verify the analysis presented in Section 4.1 , the operating temperature of $70{ }^{\circ} \mathrm{C}$, anode and cathode relative humidities of $90 \%$ and current density of $0.227 \mathrm{~A} \cdot \mathrm{cm}^{-2}(0.7 \mathrm{~V})$ is considered as the starting point. The Nyquist plot predicted by the presented equivalent circuit is fitted to the measured impedance (with the discrepancy less than 5\%) and the unknown parameters are estimated. The measured impedance and the best fit are presented in Figure 4.4. Table 4.1 presents the values of the parameters obtained from the best fit, while the physical properties of the cell and the constants used in the model are listed in Table 4.2. For these parameters, the 
same values are used for all the following figures unless it is mentioned. As an example, Figure 4.5 compares the measured Nyquist plots against the predicted values. To determine the predicted impedances in this figure, all the parameters are kept constant and only the temperature and the current density are changed to the $65^{\circ} \mathrm{C}$ and $0.264 \mathrm{~A} \cdot \mathrm{cm}^{-2}(0.7 \mathrm{~V})$ for Figure 4.5.a, and $75^{\circ} \mathrm{C}$ and $0.253 \mathrm{~A} \cdot \mathrm{cm}^{-2}(0.7 \mathrm{~V})$ for Figure 4.5.b. As it can be seen in the figure, the model can predict the measured impedances extremely well (with the discrepancy less than $1 \%$ ).

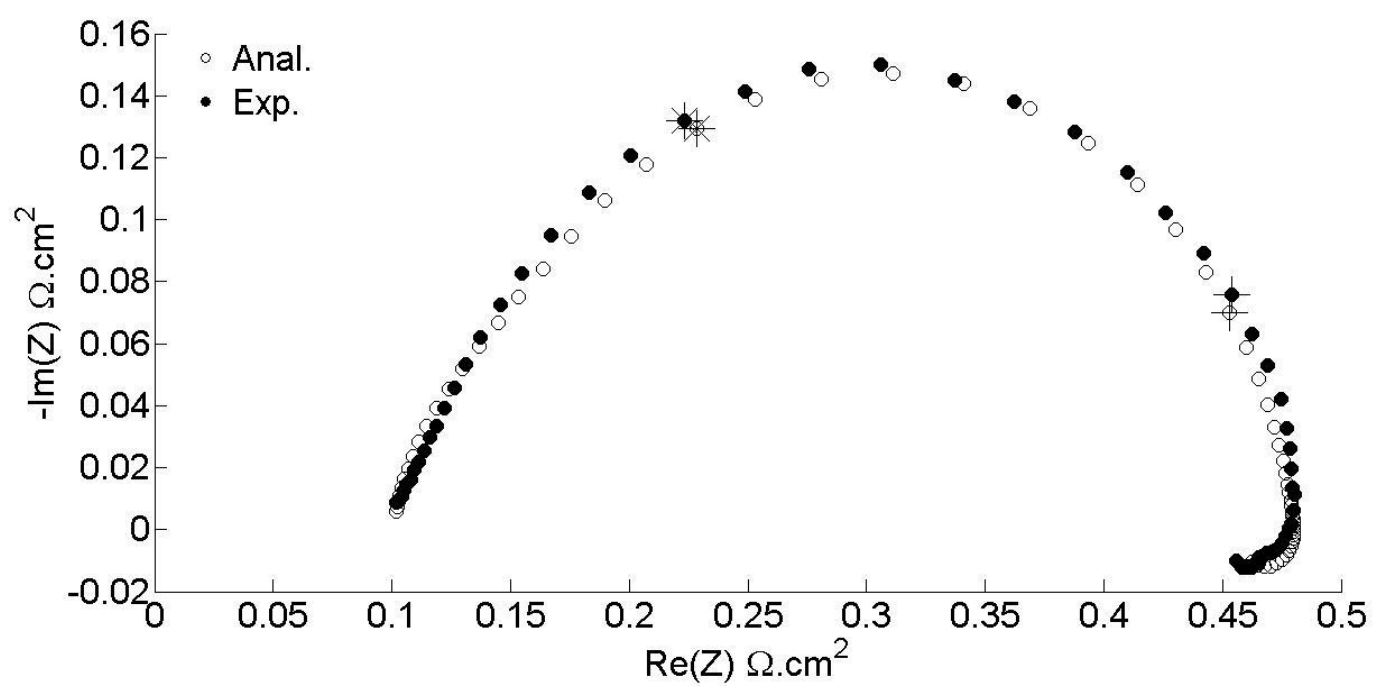

Figure 4.4 The measured and fitted Nyquist plots at $T=70^{\circ} \mathrm{C}, \mathrm{RH}_{A}=\mathrm{RH}_{C}=90 \%$, and $j=0.227 \mathrm{~A} \cdot \mathrm{cm}^{-2}$. The points corresponding to the frequencies of $10 \mathrm{~Hz}$ and $100 \mathrm{~Hz}$, are marked by ' +' and ' * ', respectively.

Table 4.1 Values of parameters obtained from the best fit presented in Figure 4.4

\begin{tabular}{llcr} 
Property & Variable & Dimension & Value \\
\hline Anode exchange current density & $j_{0, A}$ & $\mathrm{~A} \cdot \mathrm{m}^{-2}$ & $3 \mathrm{e} 3$ \\
\hline Power of the CPE for the anode & $n_{\mathrm{CPE}, A}$ & - & 0.9 \\
\hline Power of the CPE for the cathode & $n_{\mathrm{CPE} C}$ & - & 0.9 \\
\hline Magnitude of the CPE for the anode & $C_{d l, A}$ & $\mathrm{~F} \cdot \mathrm{m}^{-2}$ & 140 \\
\hline Magnitude of the CPE for the cathode & $C_{d l, C}$ & $\mathrm{~F} \cdot \mathrm{m}^{-2}$ & 170 \\
\hline Cathode transfer coefficient & $\alpha_{\mathrm{C}}$ & - & 0.35 \\
\hline Adsorbed oxygen atom concentration & $\bar{c}_{\mathrm{O}, \mathrm{s}}$ & $\mathrm{mol} \cdot \mathrm{m}^{-2}$ & $2.13 \mathrm{e}-6$ \\
\hline Diffusivity of the cathode GDL & $D_{\mathrm{GDL}, C}$ & $\mathrm{~m}^{2} \cdot \mathrm{s}^{-1}$ & $2 \mathrm{e}-5$ \\
\hline Membrane water content & $\bar{\lambda}_{\mathrm{M}}$ & - & 3.9 \\
\hline Membrane water diffusivity & $D_{\mathrm{M}}$ & $\mathrm{m}^{2} \cdot \mathrm{s}^{-1}$ & $1 \mathrm{e}-8$ \\
\hline Effective water diffusivity in cathode GDL & $D_{\mathrm{GDL}}^{\mathrm{w}}$ & $\mathrm{m}^{2} \cdot \mathrm{s}^{-1}$ & $2 \mathrm{e}-9$ \\
\hline Flooding coefficient & $f$ & - & 0.4
\end{tabular}


Table 4.2 Physical dimensions of the cell and constants used in the model

\begin{tabular}{llcr} 
Property & Variable & Dimension & Value \\
\hline Membrane thickness & $l_{\mathrm{M}}$ & $\mathrm{m}$ & $2.75 \mathrm{e}-5$ \\
\hline GDL thickness & $l_{\mathrm{GDL}}$ & $\mathrm{m}$ & $2.35 \mathrm{e}-4$ \\
\hline CL thickness & $l_{\mathrm{CL}}$ & $\mathrm{m}$ & $1.2 \mathrm{e}-5$ \\
\hline Ideal gas constant [2] & $R$ & $\mathrm{~J} \cdot \mathrm{mol}^{-1} \cdot \mathrm{K}^{-1}$ & 8.314 \\
\hline Faraday's constant [2] & $F$ & $\mathrm{C} \cdot \mathrm{mol}^{-1}$ & 96485 \\
\hline $\begin{array}{l}\text { Number of electrons } \\
\text { transferred in the reaction }\end{array}$ & $n$ & - & 2
\end{tabular}
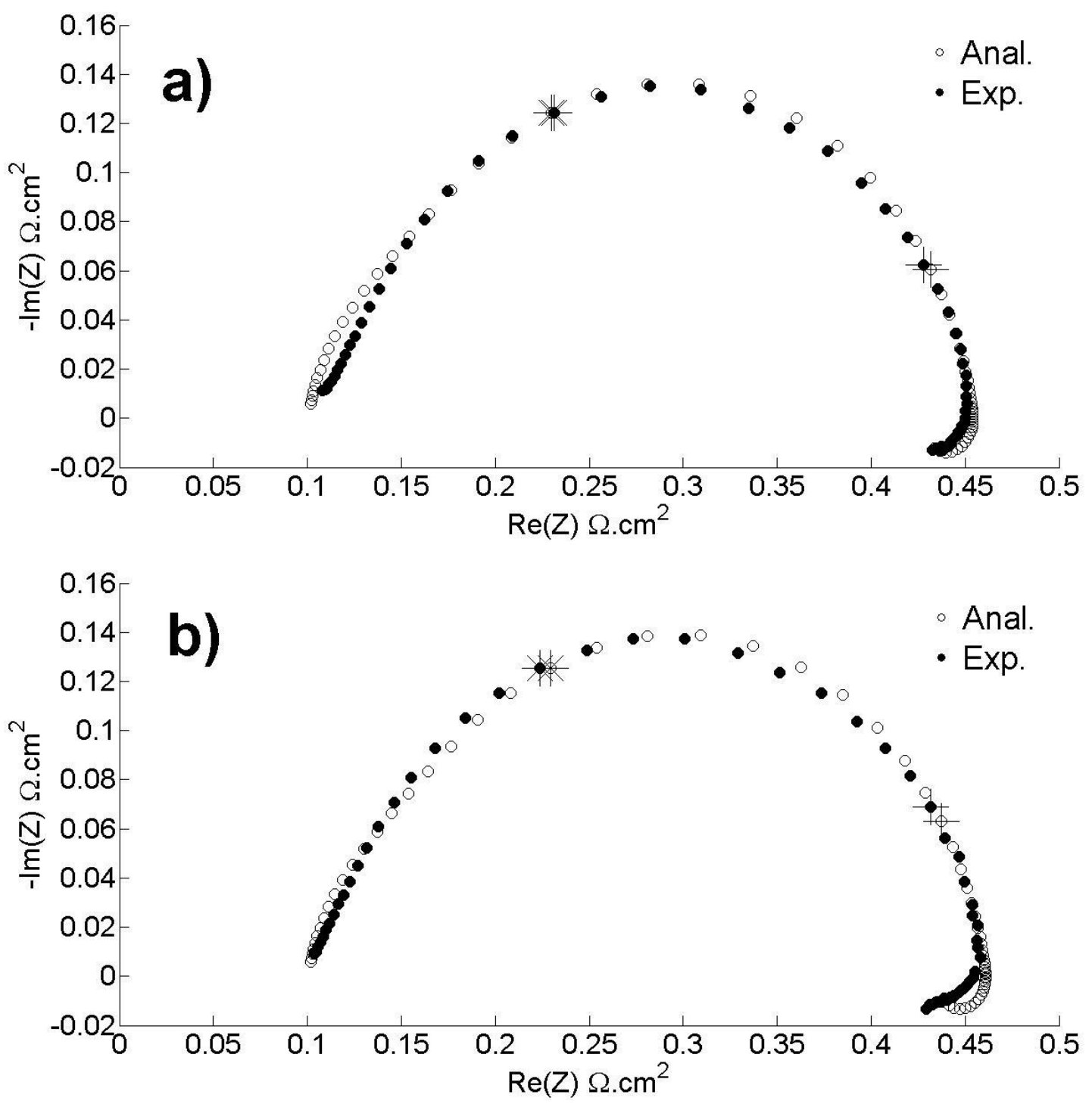

Figure 4.5 The measured and predicted impedances at (a) $T=65^{\circ} \mathrm{C}, \mathrm{RH}_{A}=\mathrm{RH}_{C}=90 \%$, and $j=0.264 \mathrm{~A} \cdot \mathrm{cm}^{-2}$ $(0.7 \mathrm{~V})$; and (b) $T=75^{\circ} \mathrm{C}, \mathrm{RH}_{A}=\mathrm{RH}_{C}=90 \%$, and $j=0.253 \mathrm{~A} \cdot \mathrm{cm}^{-2}(0.7 \mathrm{~V})$. The points corresponding to the frequencies of $10 \mathrm{~Hz}$ and $100 \mathrm{~Hz}$, are marked by '+' and '* , respectively. 


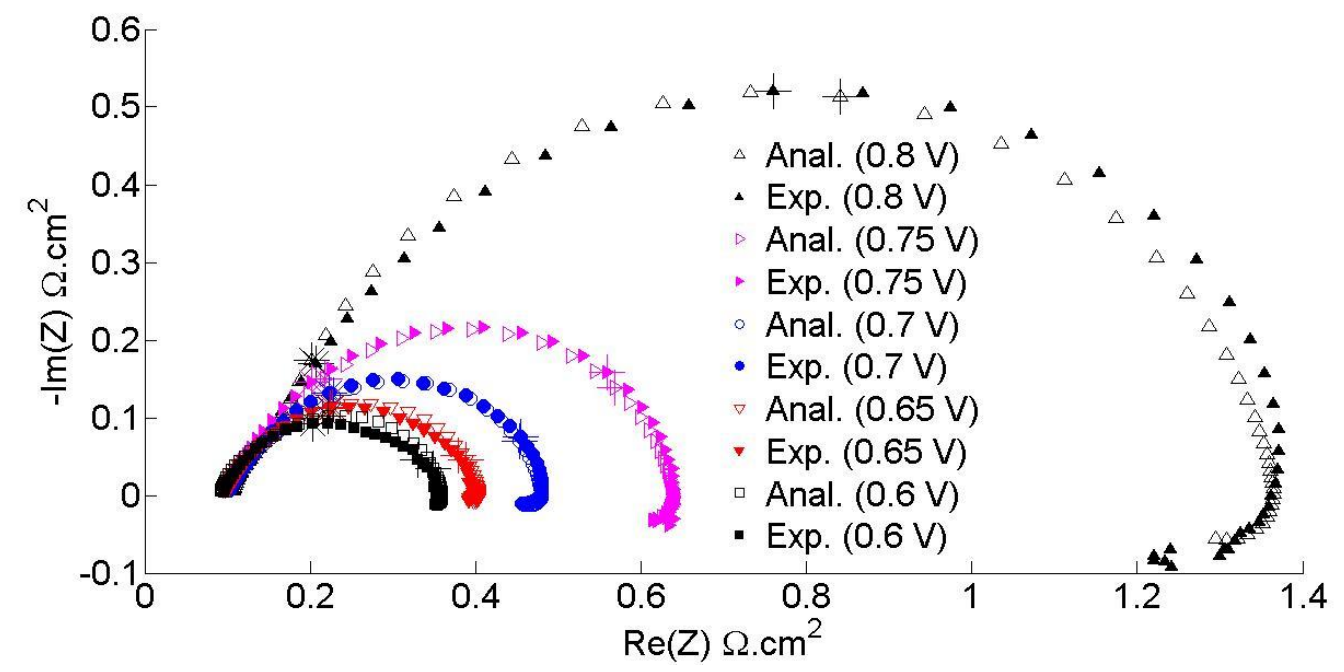

Figure 4.6 The measured and predicted impedances at $T=70^{\circ} \mathrm{C}$ and $\mathrm{RH}_{A}=\mathrm{RH}_{C}=90 \%$. The points corresponding to the frequencies of $10 \mathrm{~Hz}$ and $100 \mathrm{~Hz}$, are marked by '+' and '* ', respectively.

Figure 4.6 compares the predicted Nyquist plots against measured impedances in different current densities (for the operating temperature of $70{ }^{\circ} \mathrm{C}$, anode and cathode relative humidities of $90 \%$ ). Here again, all the parameters except for the flooding coefficient $(f)$ and effective water diffusivity in the cathode gas diffusion layer $\left(D_{\mathrm{GDL}}^{\mathrm{w}}\right)$ are kept as those listed in Table 4.1; while the corresponding current density is applied in each case. The model can predict the measured impedances extremely well in a wide range of current densities (with the discrepancy less than 4\%). As it was discussed in Chapter 2, when the current density changes considerably, the flooding coefficient $(f)$ and effective water diffusivity in the cathode gas diffusion layer ( $D_{\mathrm{GDL}}^{\mathrm{w}}$ ) have to be modified to get the best fit for the inductive loop observed in the low current densities. In essence, the increase in the current density increases the water production in the cathode catalyst layer and hence the pressure. As a result, more porous pathways are involved in the water transfer process which in fact increases the surface area used in the transport process. As the surface area is normally assumed to remain constant in the diffusion equation, this effect can be captured by enhancing the diffusivity accordingly. Furthermore, the higher rates of the water production in the catalyst layer push the water inside the gas diffusion layer. This phenomenon is reflected in the model by increasing the flooding coefficient which shows the thickness increment of the region in which the water concentration is equal to the water concentration in the catalyst layer. As mentioned, these two parameters (which are just affecting 
the inductive loops) are the only parameters which are modified as the current density is changed significantly $\left(D_{\mathrm{GDL}}^{\mathrm{w}}\right.$ and $f$ from $2 \mathrm{e}-9 \mathrm{~m}^{2} \cdot \mathrm{s}^{-1}$ and 0.4 at $0.227 \mathrm{~A} \cdot \mathrm{cm}^{-2}(0.7 \mathrm{~V})$, to $8 \mathrm{e}-11 \mathrm{~m}^{2} \cdot \mathrm{s}^{-1}$ and 0.084 at $0.0396 \mathrm{~A} \cdot \mathrm{cm}^{-2}(0.8 \mathrm{~V})$, respectively).

To verify the above-mentioned discussion, the impedance of the cell is measured in different operating conditions in other potentials $(0.6 \mathrm{~V}$ and $0.8 \mathrm{~V})$ and compared against predicted impedances. These predicted impedances are calculated using the same flooding coefficients and effective water diffusivities which used for the Nyquist plots shown in Figure 4.6 for potentials of $0.6 \mathrm{~V}$ and $0.8 \mathrm{~V}$. In other words, the impedances resulted from the same flooding coefficients and effective water diffusivities (shown in Figure 4.6) but in different temperatures and current densities are compared against the measured impedances. If the above discussion about these two parameters is right, the model must be able to capture successfully the measured impedances in different operating conditions (temperatures, relative humidities and current densities) using the values used in Figure 4.6.
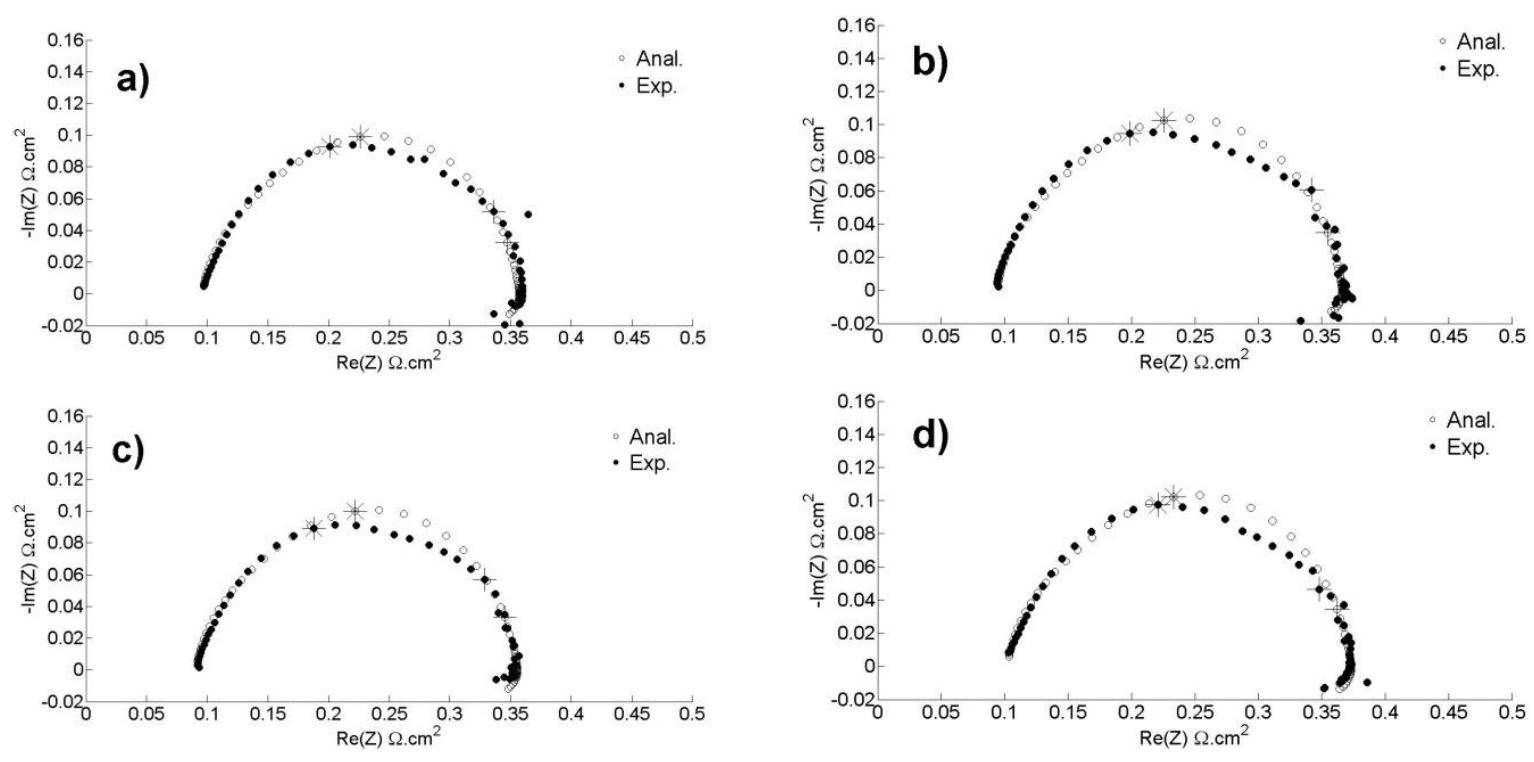

Figure 4.7 The measured and predicted impedances at (a) $T=65^{\circ} \mathrm{C}, \mathrm{RH}_{A}=\mathrm{RH}_{C}=90 \%$, and $j=0.593 \mathrm{~A} \cdot \mathrm{cm}^{-2}$ (0.6 V); (b) $T=75^{\circ} \mathrm{C}, \mathrm{RH}_{A}=\mathrm{RH}_{C}=90 \%$, and $j=0.569 \mathrm{~A} \cdot \mathrm{cm}^{-2}(0.6 \mathrm{~V})$; (c) $T=70^{\circ} \mathrm{C}, \mathrm{RH}_{A}=90 \%, \mathrm{RH}_{C}=100 \%$, and $j=0.595 \mathrm{~A} \cdot \mathrm{cm}^{-2}(0.6 \mathrm{~V})$; and (d) $T=70^{\circ} \mathrm{C}, \mathrm{RH}_{A}=90 \%, \mathrm{RH}_{C}=80 \%$, and $j=0.549 \mathrm{~A} \cdot \mathrm{cm}^{-2}(0.6 \mathrm{~V})$. The points corresponding to the frequencies of $10 \mathrm{~Hz}$ and $100 \mathrm{~Hz}$, are marked by ' +' and '* ', respectively.

To prove this point, the measured impedances in different temperatures and cathode relative humidities at $0.6 \mathrm{~V}$ are compared against the predicted ones in Figure 4.7. As it can be seen in 
this figure, the model can predict the measured impedances quite well. Similarly, Figure 4.8 compares the model predictions against the measured impedances in different temperature and anode and cathode relative humidities at $0.8 \mathrm{~V}$. This figure also proves that the model can predict the measured impedances in all different operating conditions extremely well (with the discrepancy less than $4 \%$ ).
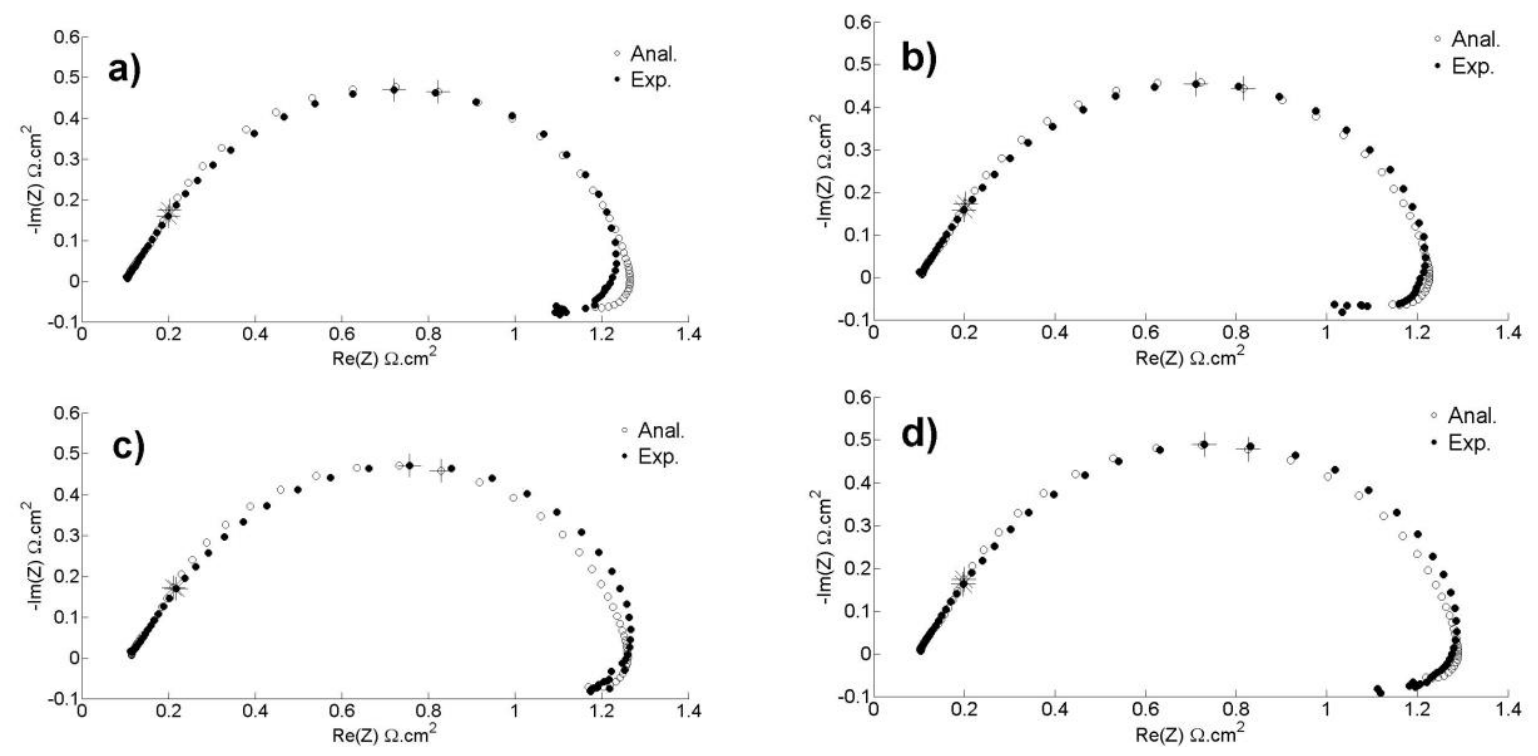

Figure 4.8 The measured and predicted impedances at (a) $T=75^{\circ} \mathrm{C}, \mathrm{RH}_{A}=\mathrm{RH}_{C}=90 \%$, and $j=0.0444 \mathrm{~A} \cdot \mathrm{cm}^{-2}$ (0.8 V); (b) $T=70^{\circ} \mathrm{C}, \mathrm{RH}_{A}=90 \%, \mathrm{RH}_{C}=100 \%$ and $j=0.0455 \mathrm{~A} \cdot \mathrm{cm}^{-2}(0.8 \mathrm{~V})$; (c) $T=70^{\circ} \mathrm{C}, \mathrm{RH}_{A}=80 \%$, $\mathrm{RH}_{C}=90 \%$, and $j=0.0442 \mathrm{~A} \cdot \mathrm{cm}^{-2}(0.8 \mathrm{~V})$; and (d) $T=70^{\circ} \mathrm{C}, \mathrm{RH}_{A}=100 \%, \mathrm{RH}_{C}=90 \%$, and $j=0.0425 \mathrm{~A} \cdot \mathrm{cm}^{-2}$ $(0.8 \mathrm{~V})$. The points corresponding to the frequencies of $10 \mathrm{~Hz}$ and $100 \mathrm{~Hz}$, are marked by ' + ' and ' * ', respectively.

\subsection{Discussions}

The measured Nyquist plots of the PEM fuel cells in different operating conditions reported in literature include one arc [112, 182], two distinguishable arcs [20, 24, 34, 66, 91, 112, 183-189], or three clearly distinguishable arcs $[15,20,46,114,190]$. If the time constants of two adjacent arcs are close, they become convoluted and make one arc. In some cases, the Nyquist plot also includes an inductive loop in the low frequency region $[15,91,106,114,186]$. As it is shown in Figure 4.9, the equivalent circuit obtained here can cover all these different cases.

Different parts of the Nyquist plot can be interpreted using the model which discussed in the following sub-sections. It has to be mentioned that although the model can also cover the 
negative real-part of the impedances in low frequencies, which are measured and reported in literature before [79, 191], this analysis is not discussed here.

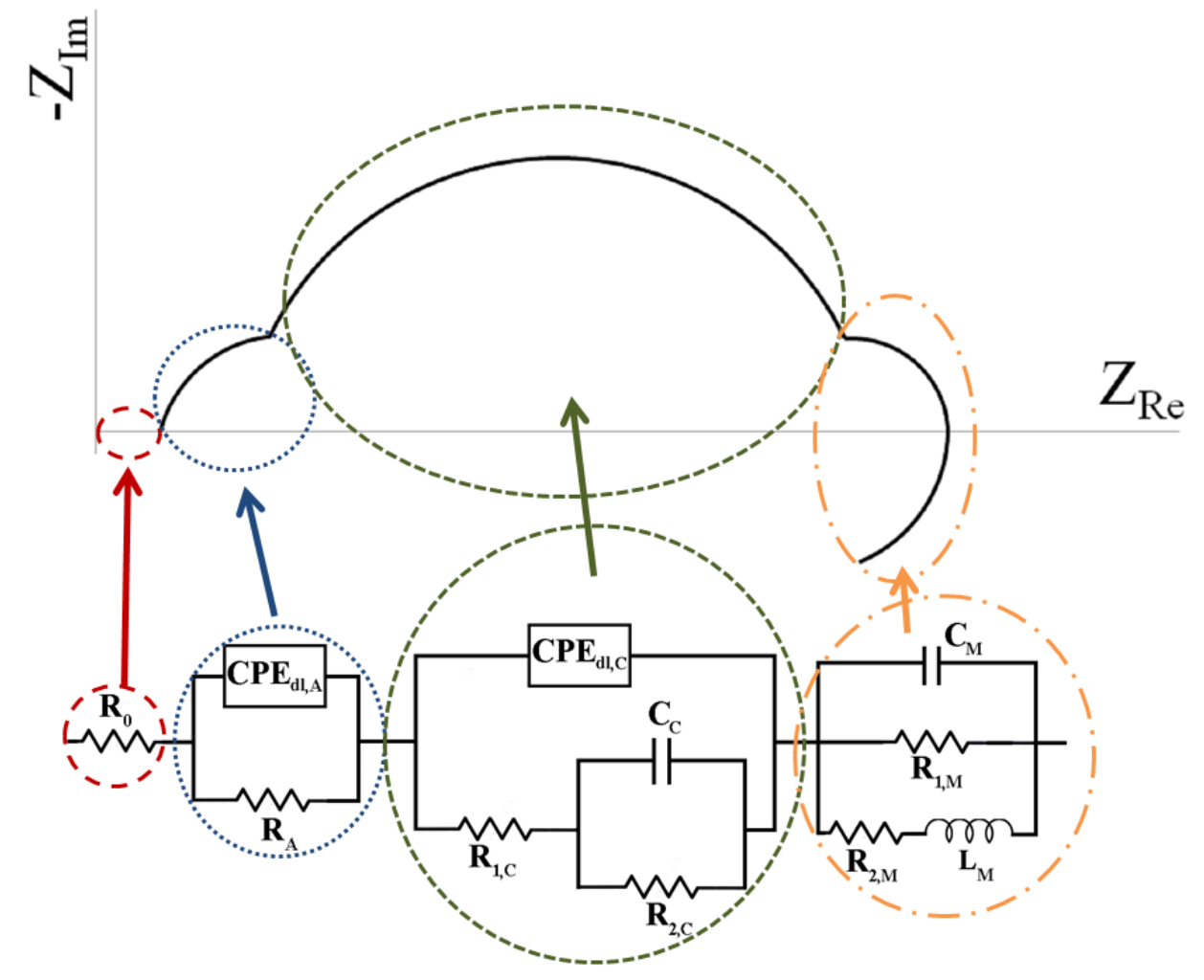

Figure 4.9 The schematic of the Nyquist plot of the PEM fuel cell and the related blocks of the equivalent circuit obtained

\subsubsection{High frequency resistance}

The intersection of the Nyquist plot with the real axis in high frequencies is called high frequency resistance (HFR). This resistance is calculated and determined here as $R_{0}$. Equation (4.17) shows that this resistance is a linear function of the membrane thickness and inversely proportional to the membrane electrical conductivity. It is reported in literature that an increase in the clamping torque applied to the cell can decrease this resistance $[66,112]$. This could be the result of a slight decrease in the membrane thickness decreasing the $R_{0}$ value as predicated by the model.

The membrane electrical conductivity is a linear function of the membrane water content, and hence, if the membrane water content increases, it is expected that $R_{0}$ and so the HFR decreases. In general, the model predicts that any changes in the operating conditions (e.g., a drastic change in the relative humidity, flooding, and dehydration) resulting in the change of the membrane 
water content has to change HFR. In fact, the measured impedances in the literature prove this point: e.g., It has been shown [20, 189] that when the relative humidity of the input gases increases drastically, the HFR clearly decreases (it has to be mentioned that for the measured impedances reported in Section 4.3, the change in HFR was not considerable as the relative humidity changes were not substantial). Another type of examples is the impedance measurements reported in $[24,91,106,182]$ showing that HFR is larger when the cell is dehydrated and smaller in flooding cases. Also, it has been reported that the HFR decreases during the break-in process in which the membrane of a fuel cell stack is hydrated to achieve the peak performance [112].

Furthermore, as the coefficients of the membrane conductivity versus water content (i.e., parameters $a$ and $b$ ) are temperature-dependent, it is expected that the HFR changes as the temperature considerably changes which is in agreement with the reported measurements [189]. The effect of different parameters discussed above on the HFR is summarized in Table 4.3.

Table 4.3 The effect of different parameters on HFR

\begin{tabular}{|c|c|c|c|c|c|c|c|}
\hline \multirow{3}{*}{$\begin{array}{c}\begin{array}{c}\text { Research } \\
\text { Group }\end{array} \\
\text { Asghari et } \\
\text { al. [23] }\end{array}$} & \multirow{2}{*}{$\begin{array}{c}\text { Conditions } \\
\text { Torque }(\mathrm{N} \cdot \mathrm{m})\end{array}$} & \multicolumn{6}{|c|}{ Values } \\
\hline & & 5 & 10 & 15 & 20 & 25 & \\
\hline & $\operatorname{HFR}\left(\Omega \cdot \mathrm{cm}^{2}\right)$ & 0.15 & 0.14 & 0.13 & 0.12 & 0.12 & \\
\hline \multirow{2}{*}{$\begin{array}{c}\text { Chang et al. } \\
\text { [59] }\end{array}$} & $\begin{array}{c}\text { Compression } \\
\text { ratio }(\%) \\
\end{array}$ & 10 & 15 & 20 & 25 & 30 & 35 \\
\hline & $\operatorname{HFR}(\Omega)$ & 0.013 & 0.010 & 0.0084 & 0.0071 & 0.0056 & 0.0051 \\
\hline \multirow{2}{*}{$\begin{array}{c}\text { Danzer and } \\
\text { Hofer [12] }\end{array}$} & $\begin{array}{l}\text { Dew Point } \\
\text { Temp }\left({ }^{\circ} \mathrm{C}\right)\end{array}$ & 28 & 35 & 45 & 55 & & \\
\hline & HFR $(\mathrm{m} \Omega)$ & 6.0 & 5.6 & 5.0 & 4.5 & & \\
\hline \multirow{2}{*}{$\begin{array}{c}\text { Yan et al. } \\
{[66]}\end{array}$} & $\mathrm{RH}(\%)$ & 20 & 40 & 60 & 80 & 100 & \\
\hline & $\operatorname{HFR}\left(\Omega \cdot \mathrm{cm}^{2}\right)$ & 4.5 & 4.0 & 3.7 & 3.4 & 3.3 & \\
\hline \multirow{2}{*}{$\begin{array}{c}\text { Fouquet et } \\
\text { al. [22] }\end{array}$} & Condition & \multirow{2}{*}{\multicolumn{2}{|c|}{$\begin{array}{c}\text { Dry } \\
0.0082 \\
\end{array}$}} & \multirow{2}{*}{\multicolumn{2}{|c|}{$\begin{array}{c}\text { Normal } \\
0.0045\end{array}$}} & \multicolumn{2}{|c|}{ Flooded } \\
\hline & $\operatorname{HFR}(\Omega)$ & & & & & & \\
\hline \multirow{2}{*}{$\begin{array}{c}\text { Kadyk et al. } \\
{[34]}\end{array}$} & Condition & \multirow{2}{*}{\multicolumn{2}{|c|}{$\begin{array}{c}\text { Dry } \\
0.021 \\
\end{array}$}} & \multirow{2}{*}{\multicolumn{2}{|c|}{$\begin{array}{c}\text { Normal } \\
0.014 \\
\end{array}$}} & \multirow{2}{*}{\multicolumn{2}{|c|}{$\begin{array}{c}\text { Flooded } \\
0.0072 \\
\end{array}$}} \\
\hline & $\operatorname{HFR}(\Omega)$ & & & & & & \\
\hline \multirow{2}{*}{$\begin{array}{c}\text { Yin and } \\
\text { Chang [58] }\end{array}$} & Condition & \multirow{2}{*}{\multicolumn{2}{|c|}{$\begin{array}{c}\text { Dry anode } \\
0.094\end{array}$}} & \multirow{2}{*}{\multicolumn{2}{|c|}{$\begin{array}{c}\text { Dry cathode } \\
0.043 \\
\end{array}$}} & \multirow{2}{*}{\multicolumn{2}{|c|}{$\begin{array}{c}\text { Normal } \\
0.035 \\
\end{array}$}} \\
\hline & $\operatorname{HFR}(\Omega)$ & & & & & & \\
\hline \multirow{2}{*}{$\begin{array}{l}\text { Schneider } \\
\text { et al. [69] }\end{array}$} & Condition & \multicolumn{3}{|c|}{ Sub-saturated } & \multicolumn{3}{|c|}{ Fully humidified } \\
\hline & $\operatorname{HFR}\left(\Omega \cdot \mathrm{cm}^{2}\right)$ & \multicolumn{3}{|c|}{0.56} & \multicolumn{3}{|c|}{0.092} \\
\hline \multirow{2}{*}{$\begin{array}{c}\text { Yan et al. } \\
{[66]}\end{array}$} & Temp. $\left({ }^{\circ} \mathrm{C}\right)$ & 30 & 40 & 50 & 60 & & \\
\hline & $\operatorname{HFR}\left(\Omega \cdot \mathrm{cm}^{2}\right)$ & 3.6 & 3.3 & 3.1 & 2.9 & & \\
\hline
\end{tabular}




\subsubsection{High frequency arc}

The HF arc is a small arc captured normally in the frequencies of $1000 \mathrm{~Hz}$ and higher in the Nyquist plots. The arc is often convoluted in the mid-frequency (MF) arc and cannot be distinguished separately (similar to the measured Nyquist plots presented in the previous section). However, it is sometimes clearly separate and distinguishable [15, 20, 46, 114, 190]. The model predicts that this arc is the result of the anode impedance and its diameter can be estimated by Equation (4.13).

The Equation (4.13) predicts that the diameter is independent of the current density or gas diffusivity. The measured Nyquist plots reported in literature show that the diameter of this arc remains unchanged in different current densities [15, 20, 46, 112, 114, 183-188, 190], temperatures [189], torques [66, 112], humidification levels [20, 189], cathode gas concentrations [34, 184], flooding or dehydration conditions [24, 91, 106, 182], starvation [189] and break-in process [112]. The impedance measurements presented in Section 4.3 also show that this arc remains unchanged in different operating temperatures, current densities and anode and cathode relative humidities. It was also shown [190] that the Nyquist plot remains unchanged in different anode stoichiometric ratios, which is again in agreement with the model.

\subsubsection{Mid-frequency arc}

The model predicts that the MF arc consists of two convoluted arcs which are governed by Equations (4.14-4.16). The $R_{1, C}$ decreases as the current density increases (Equation (4.14)) which causes the MF arc to shrink. However, in high current densities, the concentration of the absorbed oxygen atom $\left(\bar{c}_{\mathrm{O}, \mathrm{s}}\right.$, which is the required reactant of the cathode reaction) is reduced which causes the value of $R_{2, C}$ to increase (Equation (4.16)). As a result, the model predicts that by increasing the current density, the MF arc shrinks first and then inflates. The measured Nyquist plots in different current densities reported in literature [15, 20, 46, 112, 114, 183-188, 190] support this prediction. More interestingly, in the cases where the polarization curves are also reported $[46,112,114,183,184,186,188]$, it can clearly be seen that the MF arc inflation starts in the current densities at which the polarization curves enter the mass-transport region, i.e., the region in which the current drastically decreases in the polarization curve because of the mass-transport loss. It is clear that the oxygen concentration in the cathode catalyst layer has been decreased in this region which is in agreement with the predictions presented by the model. 
This trend observed in the shrinkage and inflation of the MF arc has been schematically shown in Figure 4.10.

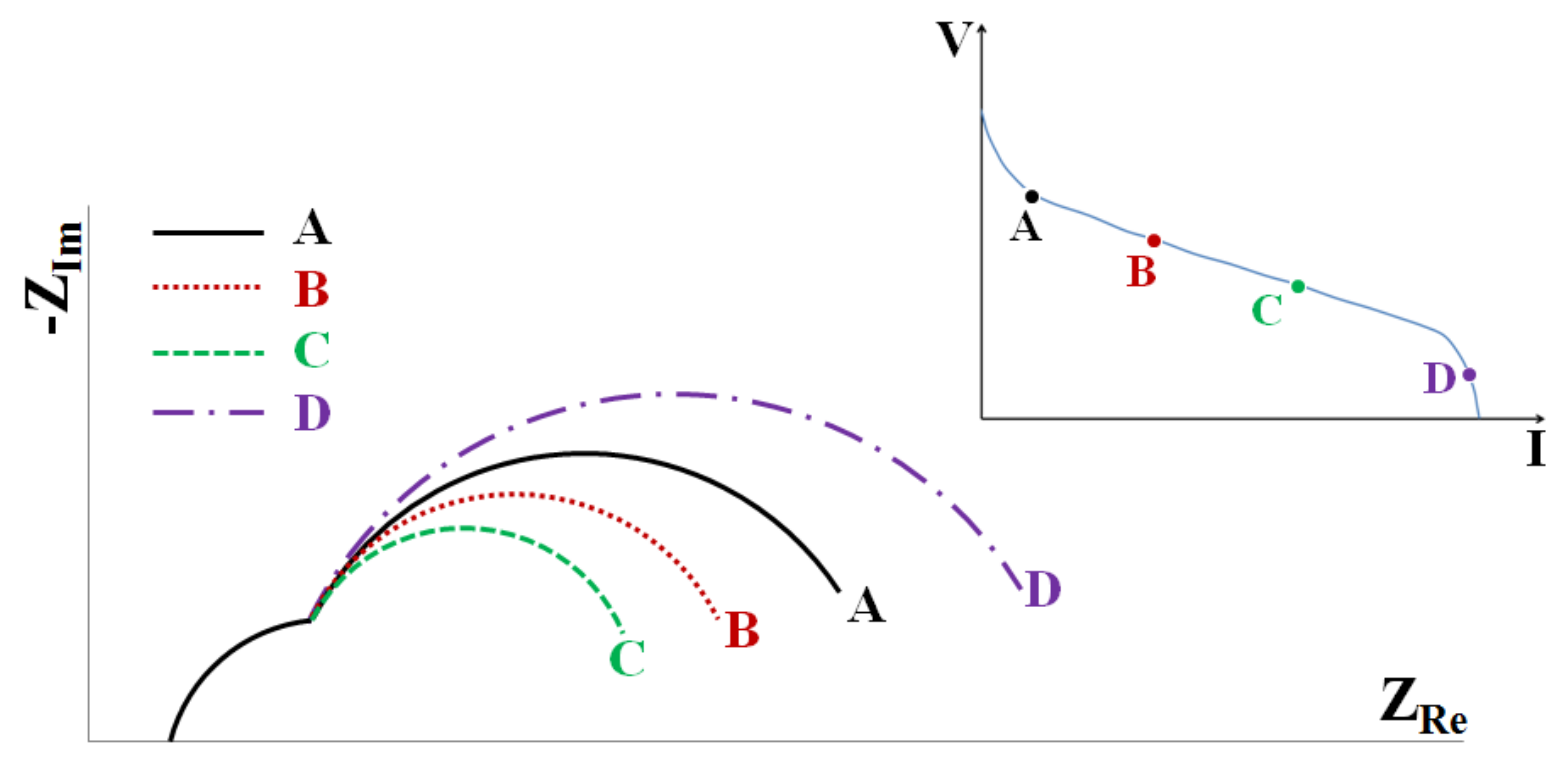

Figure 4.10 The schematic of the MF arc variations in different current densities

There are a group of impedance measurements in the literature [34, 184] showing inflation in the MF arc when air is used instead of pure oxygen. This is also in agreement with the model predictions. Clearly, the difference between the air and oxygen feeding can be applied to the model by modifying the cathode diffusion coefficient: in essence, higher diffusivity is equivalent to higher concentrations of the oxygen in the cathode catalyst layer which is conceptually equivalent to using oxygen instead of air. It was shown in Chapter 3 that the model can accurately predict these measured impedances by just adjusting the diffusion coefficient.

\subsubsection{Low frequency arc}

The LF arc is normally observed in the upper half-plane of the Nyquist plot at frequencies lower than $10 \mathrm{~Hz}$. However, in some impedance measurements, this arc is considerably small and cannot readily be separated from the MF arc (e.g., the measured impedances reported in Section 4.3). The model presented here predicts that this arc and the inductive loop are both resulted from the impedance of the membrane which is also affected by water transport in the cathode GDL. While the LF arc changes slightly as the current density or the membrane water content changes, it strongly depends on the membrane diffusion coefficient ( $D_{\mathrm{M}}$ ) which controls the $\beta$ 
parameter. The model predicts that the LF arc has an inverse relation with the membrane diffusivity: the LF arc inflates when the membrane diffusion coefficient decreases.

Various impedances measured in different operating conditions approve this conclusion. Before reviewing the experimental results, the dependency of the membrane diffusivity to the membrane water content has to be considered. It has been shown [2, 130] that the diffusion coefficient of the Nafion membrane drastically increases by the water content increase for the water content values less than 3 . It then sharply decreases as the water content increases to 5 and then gradually decreases for the larger water contents. Knowing the membrane thickness and cell temperature, the water content can directly be determined from the HF resistance (HFR) using Equation (4.17).

Asghari et al. [112] reported the impedance of the cell during the break-in process (in which the membrane is hydrated). As discussed before, the HFR continuously decreases in this process which shows the increase in the membrane water content during the break-in process. Considering the cell temperature and the membrane thickness reported in [112], the membrane water content of their cell can be estimated from the HFR value and it can be shown that the water content remains less than 3 in this process. As the water content increases during the hydration process, the membrane diffusivity has to be increased and so the LF arc must shrink. The measured impedances reported [112] confirm this prediction.

Danzer and Hofer [20] measured the cell impedance in different dew point temperatures of the supplied air. As the dew point temperature increases, the humidity level of the cell and hence the membrane water content increases, which can be captured by a continuous decrease in the HFR. Considering the values reported for the cell temperature, the membrane thickness and the surface area, the membrane water content estimated from the HFR remains less than 3 in all the cases reported. Therefore, the increase in the dew point temperature has to increase the membrane diffusivity which itself results in the shrinkage of the LF arc. The measured Nyquist plots verify this prediction.

Fouquet et al. [24] measured the Nyquist plot of flooded, normal and dry fuel cells. The water content and so diffusivity of the membrane has not changed in their flooded and normal conditions, as the HFR remained unchanged. However, the HFR in the dry condition is considerably larger which predicts the lower water content of the membrane. As the membrane diffusivity has to be smaller in this case, the model predicts that the LF arc has to be larger in the 
dry condition which is in agreement with the measured impedances reported in [24]. Schneider et al. [106] also measured the Nyquist plot in the fully humidified and sub-saturated conditions and captured similar trends.

Kadyk et al. [91] measured the cell impedance in three different humidifier temperatures representing flooding, normal and dehydration states. While the LF arc in the normal condition is smaller than the dehydrated condition (which is in agreement with the previous discussions), the flooded condition does not follow this trend and the LF arc is the largest between all these modes. However, considering the HFR value at the flooded condition and the cell temperature and the membrane thickness reported in [91], the water content can be estimated to be around 7.3. The diffusion coefficient is considerably low in the water contents as high as 7. As mentioned above, lower diffusivity is equivalent to the inflation of the LF arc, and as a result, the inflation of the LF arc in this condition is also in agreement with the model predictions.

\subsubsection{Inductive loop}

The inductive loop is the positive-imaginary part of the Nyquist plot captured in the lowest frequencies. The long measuring time required for low-frequency impedance measurements, considerably large errors associated with the measurement and even uncertainties about the consistency of the measured impedances with the Kramer-Kronig relations at very low frequencies [192] caused these frequency ranges not to be covered in most cases. Thus, this inductive loop has just been reported occasionally [e.g., 15, 91, 106, 114, 186].

Schneider et al. [106] measured the Nyquist plot (containing the inductive loop) for fullyhumidified and sub-saturated conditions. Their measurements show that the inductive loop is considerably larger in the sub-saturated condition. The model presented in this thesis predicts that this arc is related directly to the current density and inversely to the effective water diffusivity in the cathode GDL. As discussed above and also in Chapter 2, the increase in the water transfer rate in the cathode GDL increases the number of the porous pathways in the GDL for the water transfer phenomenon, increasing the real surface area involved in this phenomenon. Since the transport surface area is assumed unchanged in the diffusion equation, the diffusion coefficient needs to be modified to compensate for the increase in the surface area. As a result, the effective water diffusion coefficient has to be increased in the fully humidified condition which makes the inductive loop smaller. Therefore, the trend measured and reported by [106] is in agreement with the model predictions. 
Dale et al. [15] and Dhirde et al. [114] have reported the inductive loop in different current densities. Their measurements show that the inductive loop arc shrinks and then inflates as the current density increases. This trend can be explained by the model presented here. As mentioned before, the inductive loop is a direct function of the current density. However, by increasing the current density, the water production rate increases which results in higher water pressures in the cathode catalyst layer. As a result, the real water transfer area enhances which is covered by increasing the diffusion coefficient (as discussed in Section 4.1.2). This increase in diffusivity causes the inductive loop shrinkage. In low current densities, the diffusivity effect is dominant, and hence, the inductive loop shrinks as the current density increases. However, as the current density gradually increases, the area involved in water transfer becomes close to its limit, and hence the area and the diffusion coefficient do not change drastically. Therefore, the inductive loop is controlled only by the change in the current density. Thus, it inflates as the current density increases in high current densities.

It has to be stated that the model predicts that the time constant of the inductive loop is a direct function of the flooding parameter $f$ (which shows the part of the cathode gas diffusion layer with the same water concentration as the cathode catalyst layer). In other words, the model predicts that in the constant measuring frequency range, a smaller portion of the inductive loop can be captured when the cathode GDL is flooded. This prediction has been approved in the verification results presented in Section 4.3, where the agreement between the predicted and measured impedances in the inductive loop is shown.

Finally, Figure 4.11 reviews the discussions presented above about the HFR, the LF arc and the inductive loop. The schematics are produced based on the assumption that the membrane water content in the normal mode is less than 3 and it becomes greater than 3 in the flooded mode. The HFR continuously decreases from the dehydrated mode towards the flooded mode. The LF arc first shrinks from the dehydrated mode to the normal mode and then to a minimum in which the membrane water content reaches to 3 (not shown in the figure); then it drastically inflates as the membrane diffusion coefficient decreases. The inductive loop and its measured part (in a constant frequency range), however, continuously shrink as the cell shifts from the dehydrated mode towards the flooded mode. 


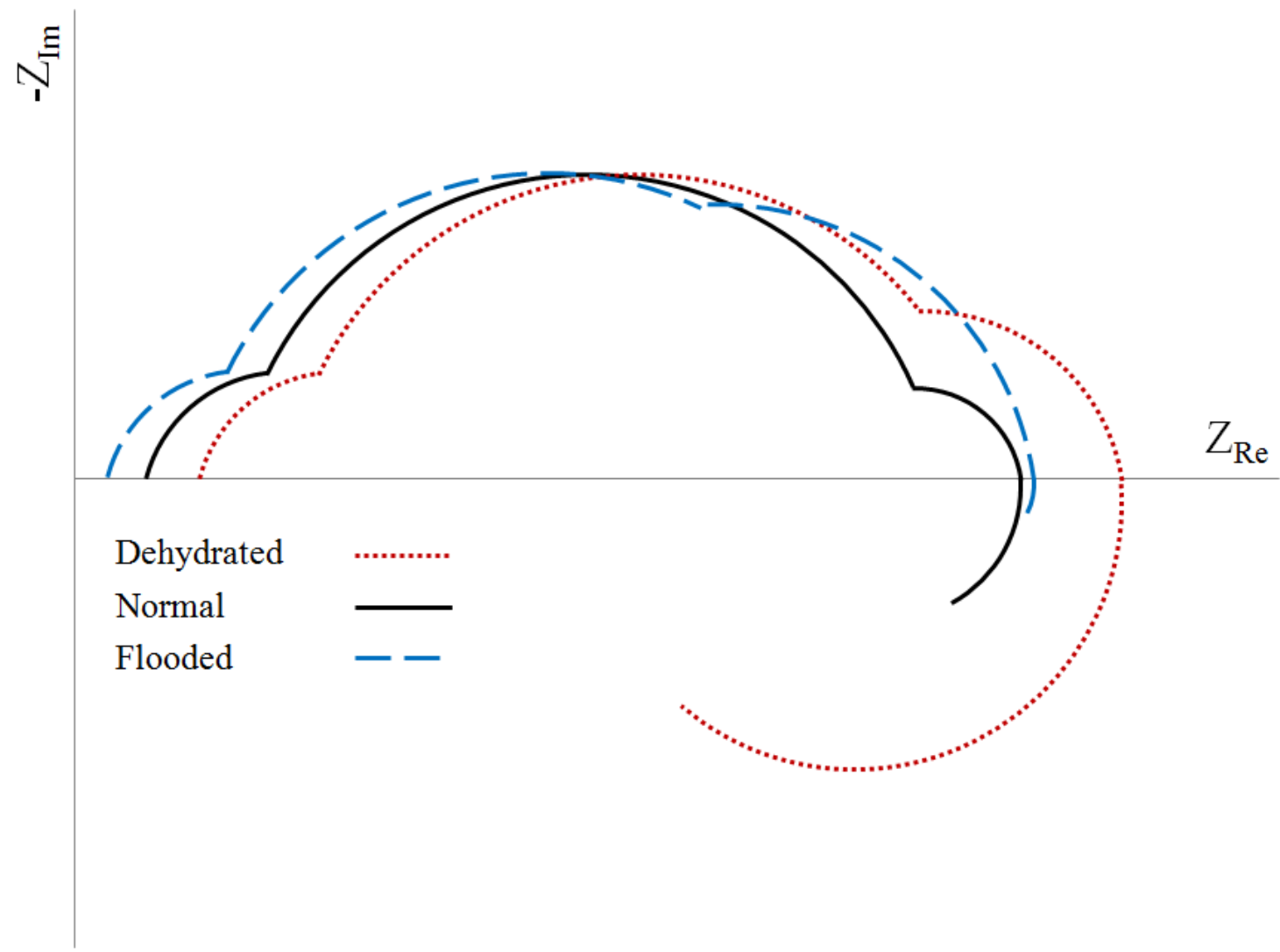

Figure 4.11 The schematic of the Nyquist plots for the normal, dehydrated, and flooded modes

\subsection{Concluding remarks}

The complete process model for the PEM fuel cell was presented in this chapter. The impedance of the cell was determined analytically and the resultant equivalent circuit was presented. The model was then compared against the cell impedances measured in different current densities, operating temperatures, and anode and cathode relative humidities. The model was able to predict the measured impedances in all different operating conditions. Next, the measured impedances and their observed trends reported in literature were compared against the model predictions. The model was also able to properly predict all these trends. As a result of this verification, the Nyquist plots of the PEM fuel cells were interpreted which can be summarised as follows: 
- The high frequency resistance (HFR), which is the intersection of the Nyquist plot with the real impedance axis, is inversely proportional to the membrane water content. HFR decreases as the hydration level of the membrane increases.

- The high frequency (HF) arc is resulted from the anode overpotential obtained from the Butler-Volmer and Nernst equations. The HF arc remains unchanged in different operating conditions, i.e., different current densities, operating temperatures, relative humidities and torques.

- The mid-frequency (MF) arc shows the cathode overpotential and shrinks as the current density increases. However, when the mass-transport loss becomes dominant and can be detected in the polarization curves, the MF arc inflates by the increase in the current density.

- The low frequency (LF) arc is inversely proportional to the membrane diffusivity. It inflates as the membrane diffusivity decreases.

- The inductive loop is directly proportional to the current density and inversely proportional to the water transport coefficient in the cathode gas diffusion layer (GDL). The capturable portion of this loop in a constant measuring frequency span, which is related to the time constant of the relevant process, is inversely related to the flooded portion of the cathode. In other words, the more the cathode GDL becomes flooded, the smaller the portion of the loop that can be captured in the same conditions. 


\section{Chapter 5. Sensitivity analysis of the impedance characteristics}

The sensitivity of the impedance characteristics to the operating conditions of the proton exchange membrane (PEM) fuel cell is studied as a sample application of the model presented in previous chapters. The measured impedances are used to estimate the indifference interval of the potential, current density, operating temperature and anode and cathode relative humidities. The indifference interval of a parameter, defined for a specific point, refers to a range out of which the impedance results are statistically dissimilar from that specific point. The analysis presented evaluates the average indifference intervals of the potential, temperature and relative humidities to be $10 \mathrm{mV}, 5{ }^{\circ} \mathrm{C}$ and $7 \%$, respectively. In other words, the changes less than the abovementioned limits do not affect the impedance of the cell statistically.

\subsection{Prelude}

The sensitivity of the measured or modeled impedances to the operating conditions of the PEM fuel cells is not well studied in the past. A few studies conducted so far for sensitivity are either based on the error analysis of the impedance measurements (as a way to diagnose the cell failure) or conducted for the sensitivity of a model with respect to the model parameters. Roy and Orazem [104, 193] studied the standard deviation of the measured impedances. They concluded that the stochastic errors are roughly independent of the measuring apparatus used for impedance measurements. They reported that the inductive loop in low frequencies is consistent with the Kramer-Kronig relations, and the impedances in this frequency range have to be associated with the physical phenomena inside the cell and not with the errors. Also, they found that the standard deviation of the measured impedances in low frequencies in the flooding state 
becomes much larger than the normal state, and hence, this standard deviation can be considered as the flooding indicator in the PEM fuel cells. Gerteisen et al. [194] have presented an analytical model to study the impedance characteristics of PEM fuel cells. To find the dominant parameters in the model, they then analyzed the sensitivity of different parts of the predicted impedances with respect to the variations of the model input values in low and high current densities. Other efforts have been made towards the study of the sensitivity of the models with respect to the model parameters. However, these efforts use the polarization curve as a tool for the sensitivity analysis. For instance, Tao et al. [195, 196] presented a numerical model to analyze the sensitivity of the polarization curve to the physicochemical properties (e.g., exchange current density) of the cell. Based on the sensitivity of the polarization curve to the changes of the model parameters, they categorized these parameters into insensitive, sensitive and highly sensitive categories. They also showed that different sets of the values of the parameters can result in similar polarization curves. Two conclusions can be obtained from their results: 1) the polarization curve is not an appropriate tool for verification of their model, and 2) the polarization curves, in general, do not thoroughly represent the physical and chemical conditions inside the cell. In other words, it is probable that two different cells working in different operating conditions produce similar polarization curves. As a result, the polarization curve is not an ideal tool for the sensitivity analysis or model verifications. To rectify the issue concerning the polarization curve, Tao et al. [195, 196] presented a three-step approach to validate their numerical model. However, the sensitivity of the cell to its operating condition was still not determined in their study. Similar analysis of the polarization curve has been conducted by Correa et al. [197] which resulted in similar sensitivity categories of the parameters of the model.

This chapter discusses the sensitivity of the impedance characteristics of the PEM fuel cells to the variations of the operating conditions. Namely, the sensitivity of the measured Nyquist plots with respect to the variations of the parameters including potential, current density, temperature, and anode and cathode relative humidities is studied. The main motivation of this work is to estimate the minimum variations in the above parameters which can be statistically traced from the measured impedances. As a result of this study, an "indifference interval" is defined for each parameter. This interval is defined as a range for which the changes in a parameter do not statistically affect the impedance of the cell or traced by the impedance measurements. The 
indifference intervals estimated here can be used to determine the required accuracy in controlling the operating conditions.

\subsection{Results and discussions}

The measured impedances of the cell in the potentials of $0.85 \mathrm{~V}, 0.8 \mathrm{~V}, 0.75 \mathrm{~V}, 0.7 \mathrm{~V}, 0.65 \mathrm{~V}$, $0.6 \mathrm{~V}$ and $0.55 \mathrm{~V}$, the operating temperatures of $65^{\circ} \mathrm{C}, 70{ }^{\circ} \mathrm{C}$ and $75{ }^{\circ} \mathrm{C}$, and the anode and cathode relative humidities of $80 \%, 90 \%$ and $100 \%$ reported and used for model verifications in Chapter 4 are used here for sensitivity analysis. In each point, the impedance is measured three times to calculate the average and the standard deviation. The frequencies lower than $1 \mathrm{~Hz}$ are not considered here for the sensitivity analysis as normally the standard deviation of the measured impedances in this range is considerably larger [192] which could affect the analyses.

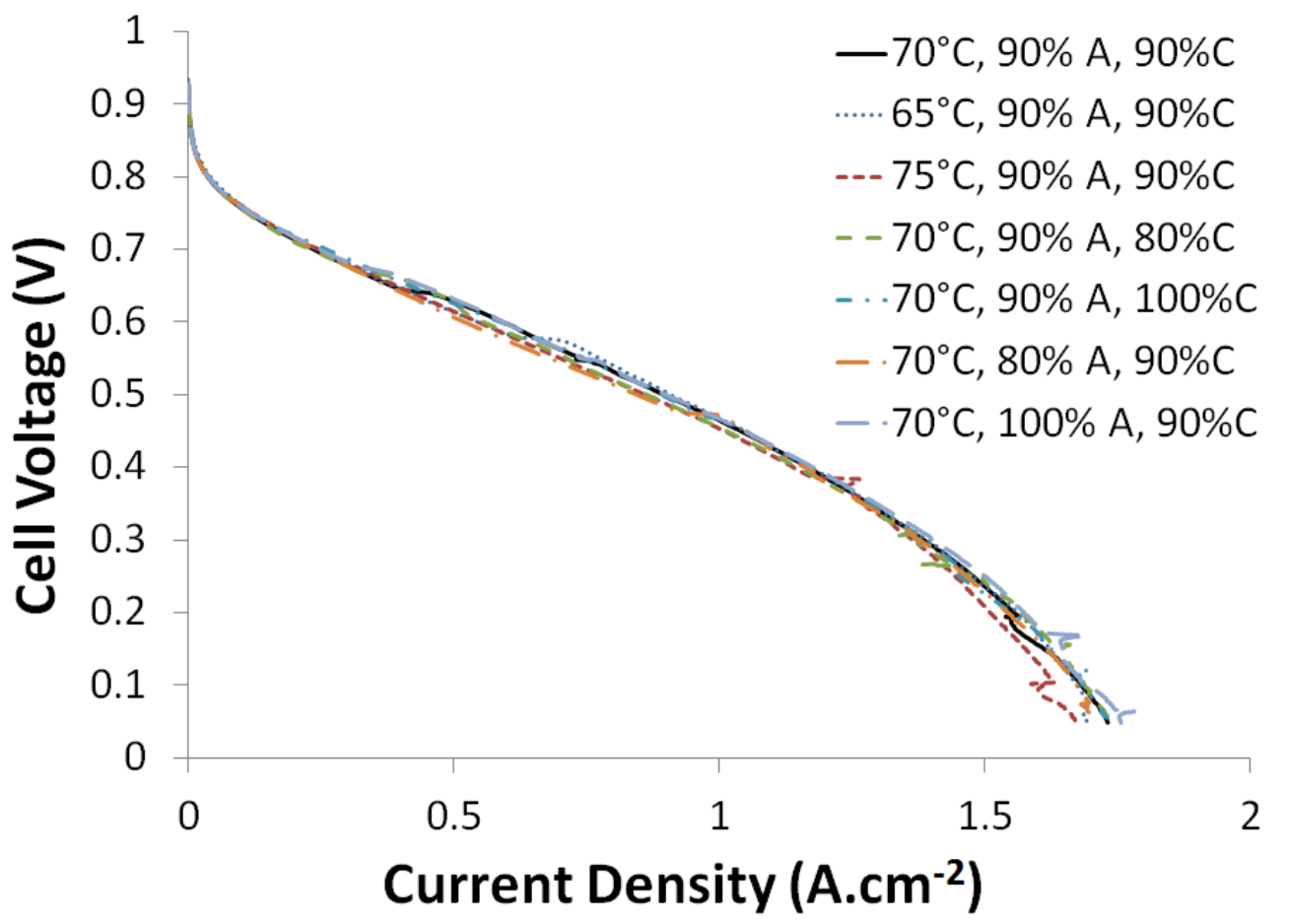

Figure 5.1 The polarization curves of the cell measured in different operating temperatures and anode and cathode relative humidities.

The measured polarization curves for the above conditions are shown in Figure 5.1. The figure shows that the polarization curves are quite similar and indistinguishable, and hence, cannot be considered for the sensitivity analysis. Figure 5.2 shows the average of three successive measurements of the Nyquist plots in different potentials. The error bars depict the deviations in 
the values of the real and imaginary parts of the measured impedance in each frequency. The figure shows that the measured impedances in successive potentials are statistically different. This conclusion is supported by calculating an indifference interval which is defined as the range in which the change in a parameter (e.g., the potential change) does not statistically affect the measured impedance. For instance, for the impedances measured in the successive potentials of $0.85 \mathrm{~V}$ and $0.8 \mathrm{~V}$, if the indifference interval of the potential is smaller than $0.05 \mathrm{~V}$, the measured impedances in the potentials of $0.85 \mathrm{~V}$ and $0.8 \mathrm{~V}$ are statistically different.

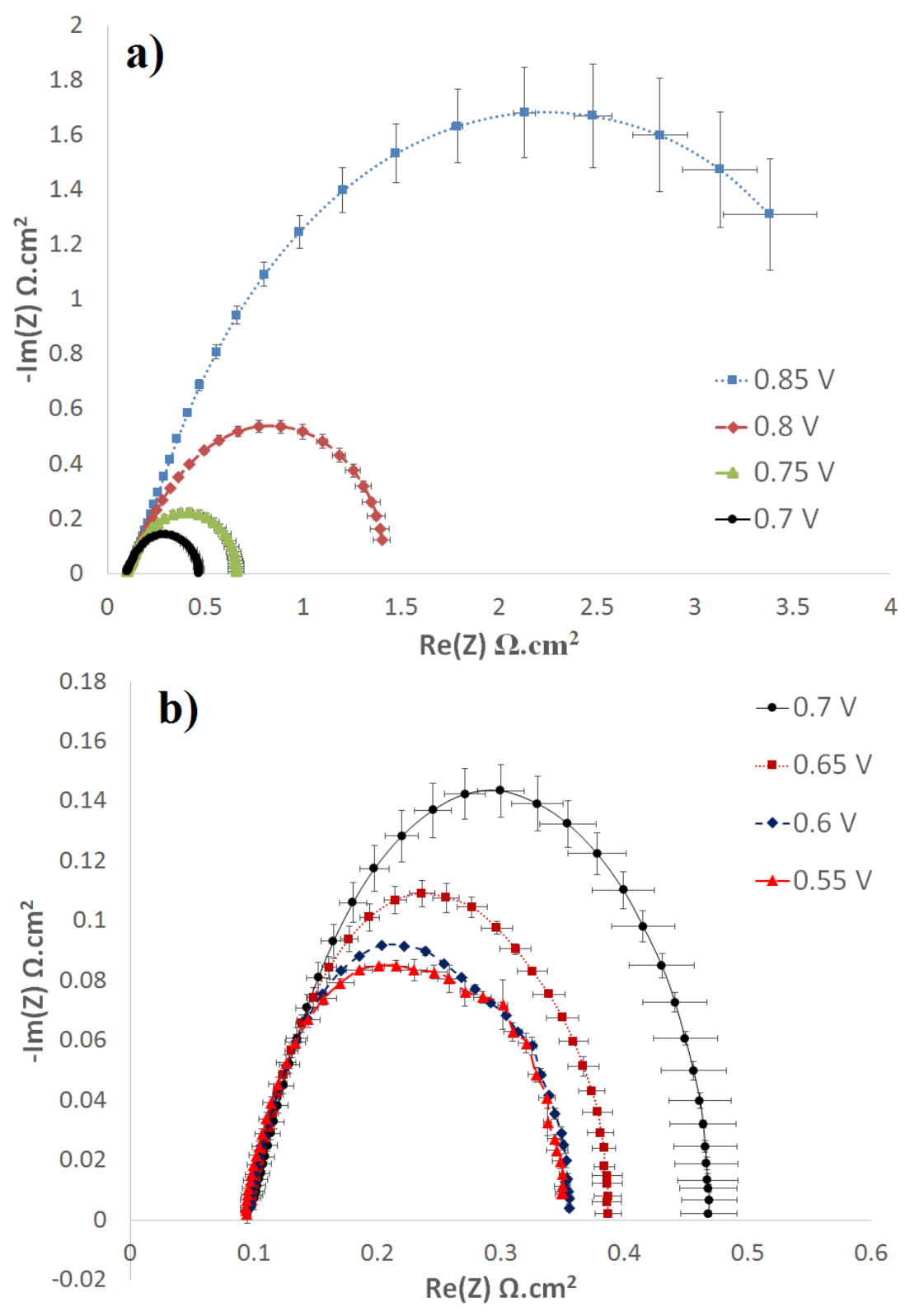

Figure 5.2 The measured Nyquist plots in the temperature of $T=70{ }^{\circ} \mathrm{C}$, the anode relative humidity of $\mathrm{RH}_{A}=90 \%$, and the cathode relative humidity of $\mathrm{RH}_{C}=90 \%$. The error bars depict the deviations in three successive measurements. The potential range of (a) $0.85 \mathrm{~V}-0.7 \mathrm{~V}$, (b) $0.7 \mathrm{~V}-0.55 \mathrm{~V}$. 
To calculate the indifference interval for the potential, it is assumed that the real and imaginary parts of the measured impedances change linearly in each potential span. It is also possible to obtain higher order estimations by combining different potential spans. For example, each two successive potential spans (e.g., $0.85 \mathrm{~V}-0.8 \mathrm{~V}$, and $0.8 \mathrm{~V}-0.75 \mathrm{~V}$ ) can be combined to use a second order approximation. However, Figure 5.1 shows that the slope of the polarization curve and hence the voltage-current relation changes significantly in different potential spans, especially in higher potentials. As the impedance characteristics of the PEM fuel cells depend strongly on the current density (see Chapter 4), it seems more accurate to correlate the impedance variations only to the adjacent potential points, resulting in the use of the linear approximation.

Using the linear estimation, the real $(x)$ and imaginary $(y)$ values of the impedance in each frequency point can be calculated as

$$
\begin{aligned}
& x=x_{u}+\frac{x_{u}-x_{l}}{V_{u}-V_{l}}\left(V-V_{u}\right) \\
& y=y_{u}+\frac{y_{u}-y_{l}}{V_{u}-V_{l}}\left(V-V_{u}\right)
\end{aligned}
$$

where $V$ denotes the voltage, and subscripts $u$ and $l$ show the upper and lower limits, respectively. As an example, in the first potential span $(0.85 \mathrm{~V}-0.8 \mathrm{~V})$, the potentials $V_{u}$ and $V_{l}$ are $0.85 \mathrm{~V}$ and $0.8 \mathrm{~V}$, respectively. Also, $x_{u}, y_{u}, x_{l}$ and $y_{l}$ are the average values of the real and imaginary parts of the measured impedances in the upper $(0.85 \mathrm{~V})$ and lower $(0.8 \mathrm{~V})$ potential limits of the span, respectively. Using a similar estimation for the standard deviation ( $S$ ), one can find

$$
\begin{aligned}
& S_{x}=S_{x, u}+\frac{S_{x, u}-S_{x, l}}{V_{u}-V_{l}}\left(V-V_{u}\right) \\
& S_{y}=S_{y, u}+\frac{S_{y, u}-S_{y, l}}{V_{u}-V_{l}}\left(V-V_{u}\right)
\end{aligned}
$$

The relations for the real and imaginary parts of the impedance and their standard deviations in each point are obtained as functions of $V$ based on the above equations. As a result, the real and imaginary parts of the impedance can be statistically compared against the upper limit of the span using the following $t$-test relations [198-200]. 


$$
\begin{aligned}
& t_{x}=\frac{x-x_{u}}{\sqrt{\frac{S_{x}^{2}+S_{x, u}^{2}}{3}}} \\
& t_{y}=\frac{y-y_{u}}{\sqrt{\frac{S_{y}^{2}+S_{y, u}^{2}}{3}}}
\end{aligned}
$$

To find the indifference interval, a specific potential limit has to be estimated such that the absolute value of at least one of the above $t$-test values becomes greater than the critical $t$-value. In this case, the values of the real or imaginary part of the impedance in the above-mentioned potential limit and those of the upper limit potential become statistically different. In other words, the potential change from $V_{u}$ to $V$ will have an impact on the real or imaginary part of the impedance which will statistically be non-negligible. Considering the significance level of $\alpha=0.05$, the critical $t$-value can be determined as $t_{c r}=2.776$ [200]. To find the indifference interval of $\left(V-V_{u}\right)$ (where $V$ is the specific potential limit and $V_{u}$ is the upper limit potential), one can substitute Equations (5.1-5.4) into (5.5-5.6) and find [198, 199]

$$
\begin{aligned}
& \frac{t_{c r}^{2}\left(S_{x, l}-S_{x, u}\right)^{2}-3\left(x_{l}-x_{u}\right)^{2}}{\left(V_{l}-V_{u}\right)^{2}}\left(V-V_{u}\right)^{2}+\frac{2 t_{c r}^{2} S_{x, u}\left(S_{x, l}-S_{x, u}\right)}{V_{l}-V_{u}}\left(V-V_{u}\right)+2 t_{c r}^{2} S_{x, u}^{2}=0 \\
& \frac{t_{c r}^{2}\left(S_{y, l}-S_{y, u}\right)^{2}-3\left(y_{l}-y_{u}\right)^{2}}{\left(V_{l}-V_{u}\right)^{2}}\left(V-V_{u}\right)^{2}+\frac{2 t_{c r}^{2} S_{y, u}\left(S_{y, l}-S_{y, u}\right)}{V_{l}-V_{u}}\left(V-V_{u}\right)+2 t_{c r}^{2} S_{y, u}^{2}=0
\end{aligned}
$$

These equations are solved for all the points in the measured impedances in all different potential spans in the range of $0.85 \mathrm{~V}-0.55 \mathrm{~V}$. To enhance the accuracy, the above procedure for finding the potential indifference interval is conducted based on at least five neighbouring points in the Nyquist plot that have statistically different real or imaginary impedances than the upper limit point. Once the potential indifference interval is calculated, the indifference interval of the current density can be estimated using the average slope of the polarization curve (Figure 5.1) in each potential span. The indifference intervals calculated are summarized in Table 5.1. This table also clarifies the impedance part and the frequency range considered in the calculations. These frequency ranges include the five neighbouring points mentioned above. As an example, in the potential span of $0.85 \mathrm{~V}-0.8 \mathrm{~V}$, the changes less than $8.5 \mathrm{mV}$ in the potential and less than 4.9 $\mathrm{mA} \cdot \mathrm{cm}^{-2}$ in the current density cannot be statistically captured by the measured impedances. 
However, when the potential and current density changes become greater than these limits, these changes affect at least the real part of the measured impedances in the frequency range of $5 \mathrm{~Hz}-$ $2 \mathrm{~Hz}$. The table shows that the PEM fuel cell impedance is on average statistically indifferent to the potential change of $10 \mathrm{mV}$. More specifically, the impedance can never capture the potential changes less than $3.1 \mathrm{mV}$ (calculated for the potential span of $0.6 \mathrm{~V}-0.55 \mathrm{~V}$ ), and certainly captures the potential changes greater than $16.5 \mathrm{mV}$ (calculated for the potential span of $0.7 \mathrm{~V}-$ $0.65 \mathrm{~V})$. The table also depicts that the sensitivity of the impedance of the cell to the current change continuously decreases as the current density increases (except for the last potential span in Table 5.1). It has to be remembered that the sensitivity analysis and the indifference interval calculations are affected by the accuracy and sensitivity of the cell control and impedance measurement systems. While it is expected to obtain slightly different values if other controlling and measuring machines are used, the approach presented can be used as a tool to estimate the sensitivity of PEM fuel cell measuring systems in general.

Table 5.1 The calculated potential and current density indifference intervals

\begin{tabular}{c|cccc}
$\begin{array}{c}\text { Potential } \\
\text { Span }\end{array}$ & $\begin{array}{c}\text { Indifference } \\
\text { Potential }\end{array}$ & $\begin{array}{c}\text { Indifference } \\
\text { Current Density }\end{array}$ & $\begin{array}{c}\text { Impedance } \\
\text { Part }\end{array}$ & $\begin{array}{c}\text { Frequency } \\
\text { Range }\end{array}$ \\
\hline $\mathbf{0 . 8 5} \mathbf{~ V - 0 . 8 ~ V}$ & $8.5 \mathrm{mV}$ & $4.9 \mathrm{~mA} \cdot \mathrm{cm}^{-2}$ & Real & $5 \mathrm{~Hz}-2 \mathrm{~Hz}$ \\
$\mathbf{0 . 8} \mathbf{~ V - 0 . 7 5 ~ V}$ & $6.1 \mathrm{mV}$ & $9.4 \mathrm{~mA} \cdot \mathrm{cm}^{-2}$ & Imaginary & $20 \mathrm{~Hz}-8 \mathrm{~Hz}$ \\
$\mathbf{0 . 7 5} \mathbf{~ V - 0 . 7 ~ V}$ & $14.0 \mathrm{mV}$ & $34.2 \mathrm{~mA} \cdot \mathrm{cm}^{-2}$ & Imaginary & $32 \mathrm{~Hz}-13 \mathrm{~Hz}$ \\
$\mathbf{0 . 7} \mathbf{~ V - 0 . 6 5 ~ V}$ & $16.5 \mathrm{mV}$ & $48.1 \mathrm{~mA} \cdot \mathrm{cm}^{-2}$ & Imaginary & $20 \mathrm{~Hz}-8 \mathrm{~Hz}$ \\
$\mathbf{0 . 6 5} \mathbf{~ V - 0 . 6 ~ V}$ & $13.1 \mathrm{mV}$ & $55.0 \mathrm{~mA} \cdot \mathrm{cm}^{-2}$ & Imaginary & $40 \mathrm{~Hz}-16 \mathrm{~Hz}$ \\
$\mathbf{0 . 6 ~ V - 0 . 5 5 ~ V}$ & $3.1 \mathrm{mV}$ & $9.1 \mathrm{~mA} \cdot \mathrm{cm}^{-2}$ & Imaginary & $795 \mathrm{~Hz}-316 \mathrm{~Hz}$
\end{tabular}

To calculate the sensitivity of the measured impedances to the variations of the operating temperature and anode and cathode relative humidities of the cell, the second order estimation is used. While similar to the potential sensitivity analysis, the linear estimation can be used here, the second order estimation is preferred as it was found that the calculated indifference intervals (see the next paragraphs) become greater than the variable span (e.g., temperature span) in some cases which questions the credibility of the linear assumption (e.g., the indifference interval of the temperature at the potential of $0.7 \mathrm{~V}$ is found to be greater than the temperature span used here $\left(5^{\circ} \mathrm{C}\right)$. Thus, the second order assumption seems to be more accurate). 
As the current density of the cell in different operating temperatures are not exactly the same (even when the voltages are the same), the effect of the current density change on the impedance measurements at different operating conditions needs to be removed. To solve this issue, the process model presented in Chapter 4 is used. To cancel the effect of the current density change due to the change in the operating conditions, the impedances predicted by the model for a certain temperature and in different current densities (obtained from the measured impedances at two successive temperatures) are determined. Then, the measured impedances at each frequency point are modified by multiplying them by the ratio of the predicted impedances at two different current densities obtained from the model for that certain temperature. As an example, the impedances are measured at the potential of $0.8 \mathrm{~V}$ in the operating temperature of $70{ }^{\circ} \mathrm{C}$ and current density of $40 \mathrm{~mA} \cdot \mathrm{cm}^{-2}$ and also in the same potential but the operating temperature of 65 ${ }^{\circ} \mathrm{C}$ and current density of $59 \mathrm{~mA} \cdot \mathrm{cm}^{-2}$. The predicted impedances produced by the model in the current densities of $40 \mathrm{~mA} \cdot \mathrm{cm}^{-2}$ and $59 \mathrm{~mA} \cdot \mathrm{cm}^{-2}$ are determined at the temperature of $70{ }^{\circ} \mathrm{C}$. The ratios of the real and also the imaginary parts of these impedances in each frequency point in these two current densities are calculated. Then, the real and imaginary parts of the measured impedance in different frequency points in the current density of $59 \mathrm{~mA} \cdot \mathrm{cm}^{-2}$ and the operating temperature of $65^{\circ} \mathrm{C}$ are multiplied by the corresponding ratio calculated above. The results are then used for the estimation of the indifference interval for the temperature (see the following paragraphs).

As the calculation process for the indifference intervals of the operating temperature and anode and cathode relative humidities are completely similar, only the temperature analysis is presented here. To calculate the indifference interval of the operating temperature, the real and imaginary parts of the impedance has to be determined as a function of the temperature. Using the second order estimation, these impedance parts can be determined as

$$
\begin{aligned}
& x=a_{x} T^{2}+b_{x} T+c_{x} \\
& y=a_{y} T^{2}+b_{y} T+c_{y}
\end{aligned}
$$

where $x, y$, and $T$ are the real and imaginary parts of the impedance and the temperature, respectively. The coefficients $a_{x}, b_{x}, c_{x}$ and $a_{y}, b_{y}, c_{y}$ are the parabolic estimation coefficients which have to be determined from the following sets of equations: 


$$
\begin{aligned}
& \left\{\begin{array}{c}
a_{x} T_{L}^{2}+b_{x} T_{L}+c_{x}=x_{L} \\
a_{x} T_{M}^{2}+b_{x} T_{M}+c_{x}=x_{M} \\
a_{x} T_{H}^{2}+b_{x} T_{H}+c_{x}=x_{H}
\end{array}\right. \\
& \left\{\begin{array}{l}
a_{y} T_{L}^{2}+b_{y} T_{L}+c_{y}=y_{L} \\
a_{y} T_{M}^{2}+b_{y} T_{M}+c_{y}=y_{M} \\
a_{y} T_{H}^{2}+b_{y} T_{H}+c_{y}=y_{H}
\end{array}\right.
\end{aligned}
$$

In the above equations, $T_{L}, T_{M}$ and $T_{H}$ present the low, medium and high operating temperatures, which are $65^{\circ} \mathrm{C}, 70^{\circ} \mathrm{C}$ and $75^{\circ} \mathrm{C}$, respectively. Also, $x_{L}, x_{M}$ and $x_{H}$ denote the average of the real parts of the measured impedances in the above low, medium and high temperatures in each frequency point. Similarly, $y_{L}, y_{M}$ and $y_{H}$ are the equivalent parameters for the imaginary part of the impedances.

Similar estimations are considered for the standard deviations of the real and imaginary parts of the impedance.

$$
\begin{aligned}
& S_{x}=a_{S, x} T^{2}+b_{S, x} T+c_{S, x} \\
& S_{y}=a_{S, y} T^{2}+b_{S, y} T+c_{S, y}
\end{aligned}
$$

The parabolic coefficients can be similarly calculated from the following sets of equations.

$$
\begin{aligned}
& \left\{\begin{array}{l}
a_{S, x} T_{L}^{2}+b_{S, x} T_{L}+c_{S, x}=S_{x, L} \\
a_{S, x} T_{M}^{2}+b_{S, x} T_{M}+c_{S, x}=S_{x, M} \\
a_{S, x} T_{H}^{2}+b_{S, x} T_{H}+c_{S, x}=S_{x, H}
\end{array}\right. \\
& \left\{\begin{array}{c}
a_{S, y} T_{L}^{2}+b_{S, y} T_{L}+c_{S, y}=S_{y, L} \\
a_{S, y} T_{M}^{2}+b_{S, y} T_{M}+c_{S, y}=S_{y, M} \\
a_{S, y} T_{H}^{2}+b_{S, y} T_{H}+c_{S, y}=S_{y, H}
\end{array}\right.
\end{aligned}
$$

The $t$-test value for the real and imaginary parts of the impedance in each frequency point can be determined similar to Equations (5.5) and (5.6).

$$
t_{x}=\frac{x-x_{M}}{\sqrt{\frac{S_{x}^{2}+S_{x, M}^{2}}{3}}}
$$




$$
t_{y}=\frac{y-y_{M}}{\sqrt{\frac{S_{y}^{2}+S_{y, M}^{2}}{3}}}
$$

In the above two equations, the $t$-test relations are presented based on the medium temperature points (i.e., the reference point). Finally, the second order estimations for the real and imaginary parts and their standard deviations (Equations (5.9), (5.10), (5.13) and (5.14)) are inserted into the above $t$-test equations (Equations (5.17) and (5.18)) which results in the following fourthorder equations:

$$
\begin{aligned}
\left(3 a_{x}^{2}-\right. & \left.t_{c r}^{2} a_{S, x}^{2}\right) T^{4}+\left(6 a_{x} b_{x}-2 t_{c r}^{2} a_{S, x} b_{S, x}\right) T^{3} \\
& +\left(3 b_{x}^{2}+6 a_{x}\left(c_{x}-x_{M}\right)-t_{c r}^{2} b_{S, x}^{2}-2 t_{c r}^{2} a_{S, x} c_{S, x}\right) T^{2}+\left(6 b_{x}\left(c_{x}-x_{M}\right)-2 t_{c r}^{2} b_{S, x} c_{S, x}\right) T \\
& +\left(3\left(c_{x}-x_{M}\right)^{2}-t_{c r}^{2}\left(S_{x, M}^{2}+c_{S, x}^{2}\right)\right)=0 \\
\left(3 a_{y}^{2}-\right. & \left.t_{c r}^{2} a_{S, y}^{2}\right) T^{4}+\left(6 a_{y} b_{y}-2 t_{c r}^{2} a_{S, y} b_{S, y}\right) T^{3} \\
& +\left(3 b_{y}^{2}+6 a_{y}\left(c_{y}-y_{M}\right)-t_{c r}^{2} b_{S, y}^{2}-2 t_{c r}^{2} a_{S, y} c_{S, y}\right) T^{2}+\left(6 b_{y}\left(c_{y}-y_{M}\right)-2 t_{c r}^{2} b_{S, y} c_{S, y}\right) T \\
& +\left(3\left(c_{y}-y_{M}\right)^{2}-t_{c r}^{2}\left(S_{y, M}^{2}+c_{S, y}^{2}\right)\right)=0
\end{aligned}
$$

These equations are numerically solved for all frequency points. The only unknown in the above two equations is $T$, for which the closest solutions to $T_{M}$ are accepted (since the medium temperature point is the reference point in this analysis). In essence, these temperatures are the closest to $T_{M}$ which result in $t$-test values greater than $t_{c r}$. In other words, these temperatures estimate the temperature changes which statistically affect the Nyquist plots. The same analysis is repeated for all impedances measured in the potentials of $0.6 \mathrm{~V}, 0.7 \mathrm{~V}$ and $0.8 \mathrm{~V}$ and also for the anode and cathode relative humidities. The results are presented in Figures 5.3, 5.4, and 5.5.

Figure 5.3 depicts the calculated indifference intervals of the operating temperature $\left({ }^{\circ} \mathrm{C}\right)$ in the potentials of $0.8 \mathrm{~V}, 0.7 \mathrm{~V}$ and $0.6 \mathrm{~V}$. In each frequency point, the minimum of the indifference intervals calculated separately for the real and imaginary parts of the measured impedances is shown. Obviously, the smaller the indifference interval, the more sensitive the impedance in that specific frequency. The figures show that the temperature indifference interval is smaller and so the temperature sensitivity is higher in low current densities. In general, the calculated indifference intervals in different frequency points in each potential are close which can verify the consistency of the analysis. Similar analysis is performed on the measured impedances in different anode and cathode relative humidities. 

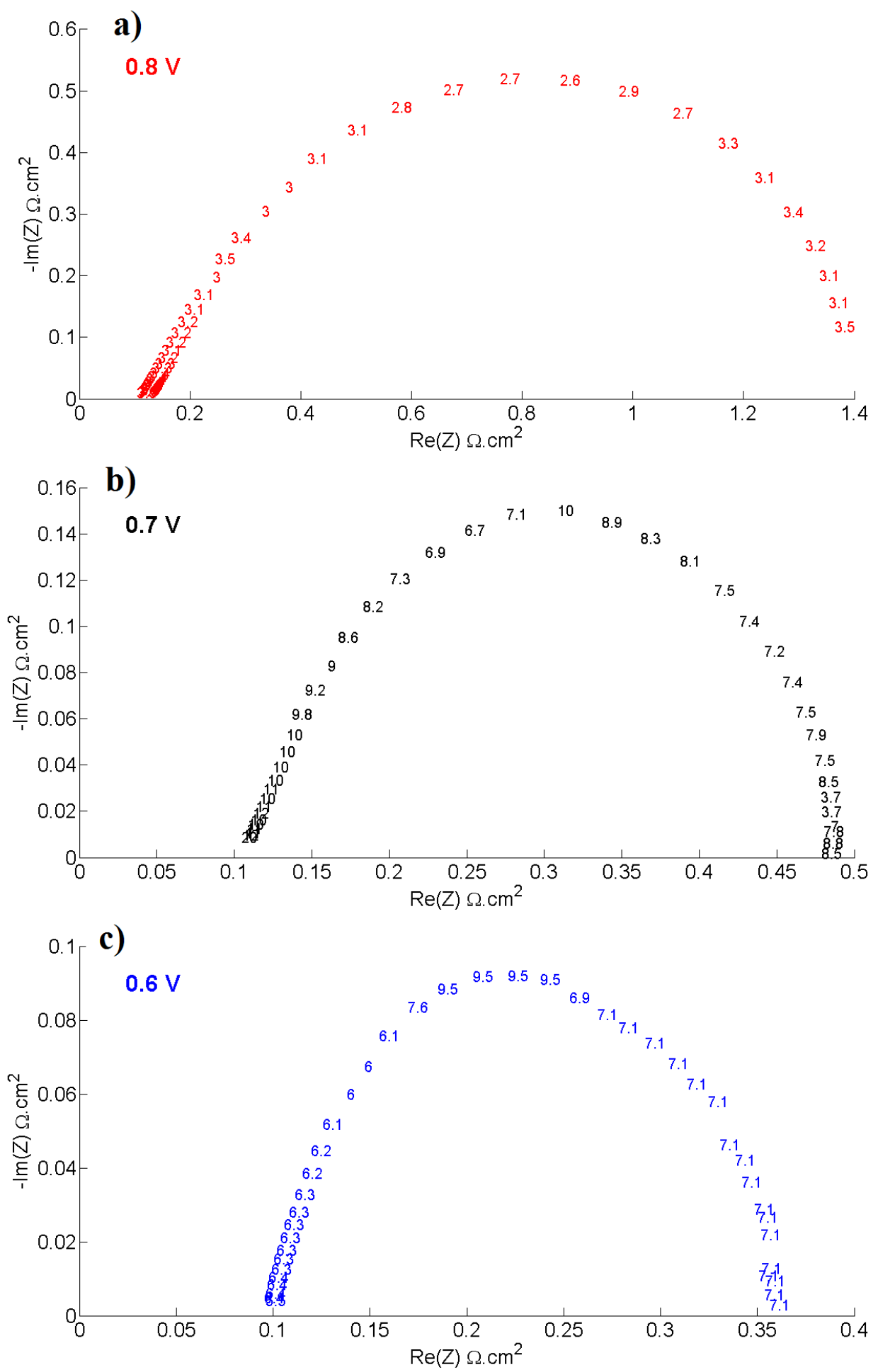

Figure 5.3 The calculated indifference intervals of the operating temperature $\left({ }^{\circ} \mathrm{C}\right)$ in different potentials;

$$
\text { (a) } 0.8 \mathrm{~V} \text {, (b) } 0.7 \mathrm{~V} \text {, (c) } 0.6 \mathrm{~V} \text {. }
$$

The indifference interval distributions in different frequency points and potentials are shown in Figures 5.4 and 5.5 for the anode and cathode relative humidities, respectively. In each point, the indifference interval of the relative humidity $(\%)$ is shown. 

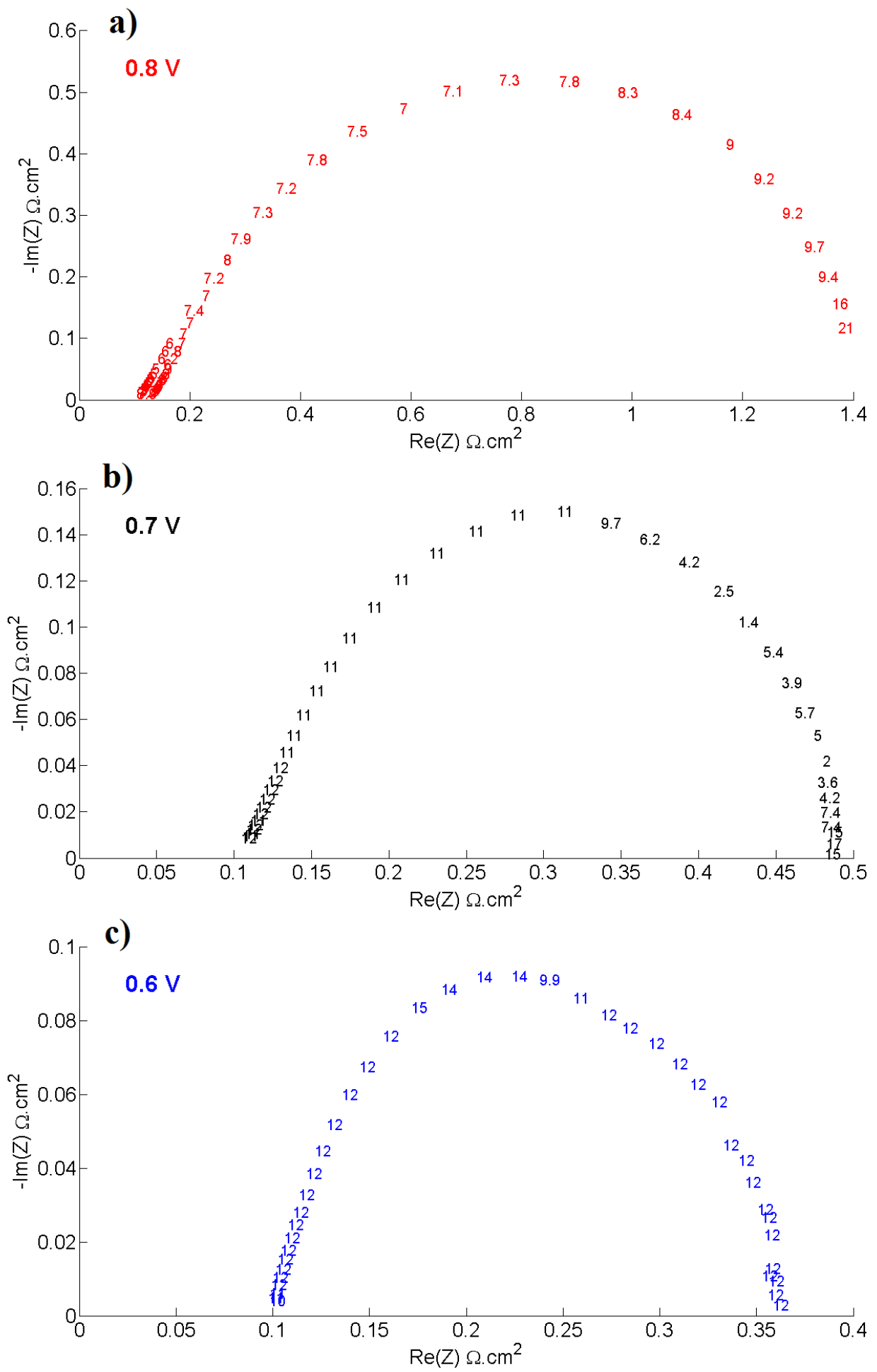

Figure 5.4 The calculated indifference intervals of the anode relative humidity (\%) in different potentials;

$$
\text { (a) } 0.8 \mathrm{~V} \text {, (b) } 0.7 \mathrm{~V} \text {, (c) } 0.6 \mathrm{~V} \text {. }
$$

As an example, the indifference interval of $10 \%$ for the anode relative humidity in a specific frequency point means that the real and imaginary part of the impedance in that point are not affected by the variations in the anode relative humidity, if these variations are less than $10 \%$. 

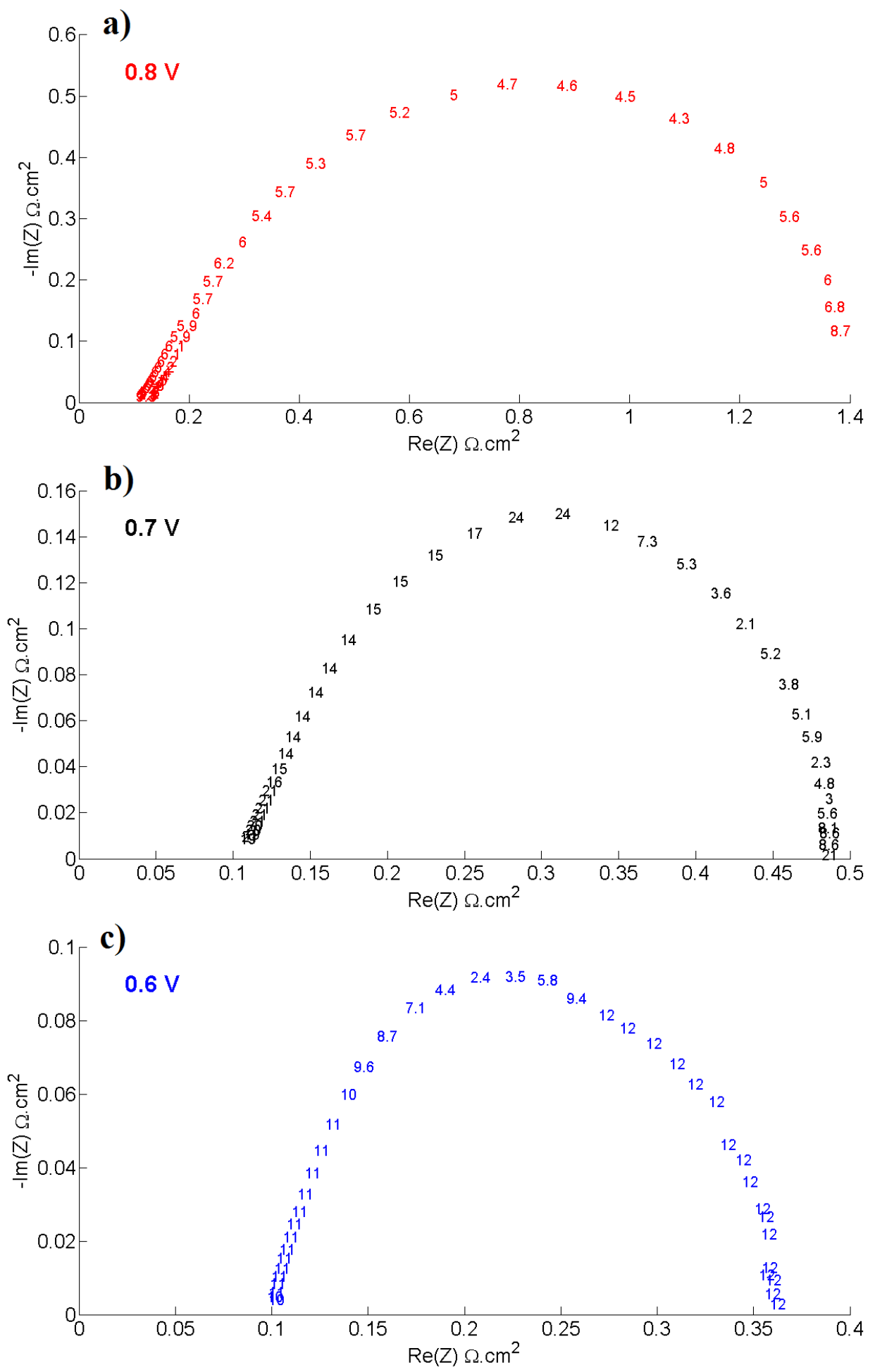

Figure 5.5 The calculated indifference intervals of the cathode relative humidity (\%) in different potentials; (a) $0.8 \mathrm{~V}$, (b) $0.7 \mathrm{~V}$, (c) $0.6 \mathrm{~V}$.

In general, Figures 5.3-5.5 show that the indifference interval is larger in the lowest frequency points. This shows that the sensitivity of the measured impedances in low frequencies is lower. This observation also supports the decision of not considering the measured impedances in 
frequencies lower than $1 \mathrm{~Hz}$ in the sensitivity analysis as discussed before. Once more it has to be remembered that the calculated indifference intervals presented here are controlled by the accuracy and sensitivity of the impedance measuring and fuel cell controlling systems used here. Thus, these values have to be considered as estimates for a nominal fuel cell controlling system.

Table 5.2 presents the overall values of the indifference intervals of the operating temperature and anode and cathode relative humidities in different potentials. The table summarizes one value of the indifference interval for each voltage and operating condition parameters (i.e., temperature, and anode and cathode relative humidities). To represent the sensitivity of the impedance measured for different operating parameters, one value among those indicated in Figure 5.3-5.5 needs to be chosen. One can practice different methods for selecting this one point. One method would be to select the highest value of the indifference interval presented in each of the Figures 5.3-5.5. Although this approach is safe (choosing the lowest sensitive point), it does not capture the overall sensitivity of the measurements. In this chapter, another approach was used (similar to the method presented for the potential sensitivity analysis). For each of the indifference interval values listed in Table 5.2, a group of successive frequency points (5 points here) were selected, and among them the largest indifference interval was chosen. Then, these chosen indifference intervals from different groups are compared and the lowest was selected and listed in Table 5.2. This value represents the minimum change which causes statistically measurable differences in at least five successive points in the impedance plot. The table shows that the indifference interval of the temperature is on average around $5{ }^{\circ} \mathrm{C}$ for three potentials studied here. In other words, it predicts that the operating temperature needs to be changed around $5{ }^{\circ} \mathrm{C}$ to affect the cell impedance statistically. The calculated indifference intervals of the temperature also predict that the temperature changes less than $2.9^{\circ} \mathrm{C}$ (which was listed for the potential of $0.8 \mathrm{~V}$ ) cannot certainly be captured by impedance characteristics, while the temperature changes greater than $7.5{ }^{\circ} \mathrm{C}$ (which was listed for the potential of $0.6 \mathrm{~V}$ ) definitely affect the impedance of the cell. The table also predicts that the indifference interval of the anode and cathode relative humidities can be considered on average as $8 \%$ and $6 \%$, respectively. It also shows that the impedance of the cell is more sensitive to the cathode relative humidity than that of the anode. Also, the minimum limit of $5 \%$ relative humidity change can be estimated as a safe range below which the effect of the changes in the relative humidity on the impedance of the cell can be neglected. 
Table 5.2 The calculated indifference intervals of the operating temperature and anode and cathode relative humidities

\begin{tabular}{ccccc} 
& Potential & $\begin{array}{c}\text { Indifference } \\
\text { Interval }\end{array}$ & $\begin{array}{c}\text { Impedance } \\
\text { Part }\end{array}$ & $\begin{array}{c}\text { Frequency } \\
\text { Range }\end{array}$ \\
\cline { 2 - 5 } Operating & $\mathbf{0 . 8 ~ V}$ & $2.9^{\circ} \mathrm{C}$ & Real & $16 \mathrm{~Hz}-6 \mathrm{~Hz}$ \\
Temperature & $\mathbf{0 . 7 ~ V}$ & $7.5^{\circ} \mathrm{C}$ & Real & $20 \mathrm{~Hz}-8 \mathrm{~Hz}$ \\
& $\mathbf{0 . 6 ~ V}$ & $6.2^{\circ} \mathrm{C}$ & Real & $500 \mathrm{~Hz}-200 \mathrm{~Hz}$ \\
\hline Anode Relative & $\mathbf{0 . 8 ~ V}$ & $5.9 \%$ & Real & $1600 \mathrm{~Hz}-630 \mathrm{~Hz}$ \\
Humidity & $\mathbf{0 . 7 ~ V}$ & $5.4 \%$ & Imaginary & $25 \mathrm{~Hz}-10 \mathrm{~Hz}$ \\
\hline Cathode Relative & $\mathbf{0 . 6 ~ V}$ & $11.7 \%$ & Real & $10 \mathrm{kHz}-3.1 \mathrm{kHz}$ \\
Humidity & $\mathbf{0 . 7} \mathbf{~ V}$ & $5.8 \%$ & Real & $10 \mathrm{~Hz}-4 \mathrm{~Hz}$ \\
& $\mathbf{0 . 6 ~ V}$ & $7.1 \%$ & Imaginary & $20 \mathrm{~Hz}-8 \mathrm{~Hz}$ \\
& & Real & $160 \mathrm{~Hz}-65 \mathrm{~Hz}$
\end{tabular}

Finally, it has to be noted that the results presented in Tables 5.1 and 5.2 show that by measuring the impedance of the cell in specific frequencies, it is possible to roughly sense the changes in the potential, current density, operating temperature, and anode and cathode relative humidities. In other words, the sensitivity analysis method presented here can be used as a tool to monitor the changes in the operating condition parameters. For example, by measuring the impedance of the cell in the range of $40 \mathrm{~Hz}-2 \mathrm{~Hz}$, if we observe that the impedance has changed statistically, we can say that the potential has changed at least by $6.1 \mathrm{mV}$ (which is the smallest indifference interval in the potential span of $0.85 \mathrm{~V}-0.6 \mathrm{~V}$ ). Similar approach has been used before for estimating the cathode flow rates based on the impedance measurements [190].

\subsection{Concluding remarks}

The sensitivity of the impedance of the PEM fuel cell to the variations of the potential, current density, operating temperature, and anode and cathode relative humidities were studied in this chapter as an application for the model presented previously in Chapter 4. Using the $t$-test, the indifference interval of each of these parameters was calculated in different potentials. The indifference interval is defined as the range in which the parameter change cannot be statistically captured by the measured impedances. It was shown that the impedance characteristics of the PEM fuel cell are on average not sensitive to $10 \mathrm{mV}$ changes in the potential, $5{ }^{\circ} \mathrm{C}$ changes in the operating temperature, and 7\% changes in the anode and cathode relative humidities. Also, 
the sensitivity of the cell impedance to the current changes continuously decreased as the current density increased. Finally, the results showed that the measured impedance of the cell can be used as a tool to sense the changes in the potential, current density, temperature and anode and cathode relative humidities. 


\section{Chapter 6. Anode and cathode starvation effects}

In this chapter, anode and cathode starvations in proton exchange membrane (PEM) fuel cells are studied using the model presented in Chapter 4. The impedances are measured while the anode and cathode feeding gasses are diluted using helium and nitrogen, respectively, and the Nyquist plots are compared against the normal operating conditions. It is shown that anode starvation does not affect the measured Nyquist plots; while cathode starvation significantly inflates the mid-frequency (MF) arc. These results are in agreement with the trends predicted from the process model (see Chapter 3).

\subsection{Prelude}

The starvation phenomenon in PEM fuel cells can be defined as a state in which there is not enough hydrogen in the anode or oxygen in the cathode. Starvation can occur if the flow rates of the feeding gasses decrease; it can also be the result of flooding that can clog the anode and cathode porous electrodes hindering the transport of the reactants to the reaction sites. The anode potential can also reach to corrosive potentials in starvation [52, 201].

Starvation and flooding phenomena can be studied using the electrochemical impedance spectroscopy (EIS) method. The effect of the flow rates of the reactants has been studied by O'Rourke et al. [190] who measured the Nyquist plots of a PEM fuel cell in different cathode and anode stoichiometry ratios. They showed that the change in the cathode stoichiometry ratio clearly affects the Nyquist plot; while the change in the anode stoichiometry does not change the plot. The effects of the anode and cathode stoichiometry were also studied by Danzer and Hofer [20]. They also reported that while the cathode stoichiometry affects the Nyquist plots, the anode stoichiometry has a minimal effect on the plot. Yan et al. [189] also reported similar effects of the cathode stoichiometry on the impedance characteristics of the cell. While these experimental 
results are normally used to study the starvation effects on the Nyquist plots, there are still debates regarding these cases. As the stoichiometry ratio of the feeding gas changes, the gas flow rates and velocity profiles are also altered which change the flow structure in the channels and also water management in the cell. Consequently, the results captured could be affected by these sideline phenomena. As a result, a new experimental approach is needed which changes the stoichiometry while keeping the flow rates and velocities unchanged. Moreover, if the channels are not dead ended, the stoichiometry is not always a reliable factor for the starvation assessment. In fact, the stoichiometry measures the ratio of the needed reactants (fuel or oxygen) in the catalyst layer to the fed reactants in the channel. Considering the complex transport

phenomena from the channel towards the catalyst layer through the gas diffusion layer (GDL), it is reasonable to expect to have (at least partial or local) starvation in the catalyst layer even when the stoichiometry is considerably high.

Another factor that can potentially lead to starvation is flooding, as mentioned before. Flooding is in fact undesirable as it causes partial or local starvation. The reported impedance characteristics obtained under the flooded and normal conditions in PEM fuel cells [e.g., 24, 91, 103-107] do not normally distinguish the flooding in the anode electrode from that in the cathode electrode. Therefore, these reported impedances cannot be used to study separately the anode and cathode starvation.

In this chapter, a new experimental approach is presented which enables measuring the impedance of a PEM fuel cell in different stoichiometry ratios in the anode and cathode while the flow rates are kept constant. To decrease the anode and cathode stoichiometry, the hydrogen and air are mixed with helium and nitrogen, respectively, and the impedances are measured and discussed. The measured impedances are then compared against the trends observed by the process model presented in Chapter 4. Similar to the process model predictions, the comparisons prove that anode starvation cannot be captured by impedance characteristics; while the cathode starvation can easily be captured from the mid-frequency (MF) arc.

\subsection{Experimental setup}

The details of the PEM fuel cell and instruments used here for impedance measurements were presented before in Chapter 4 (Section 4.2). The impedance measurement sweeps the frequency span of $10 \mathrm{kHz}-10 \mathrm{mHz}$ at the rate of 10 points per decade in the potentiostatic mode. The cell 
and the anode and cathode flow temperatures are kept at $70{ }^{\circ} \mathrm{C}$; while the relative humidities of the reactants are $90 \%$. The Nyquist plots are measured at the potentials of $0.75 \mathrm{~V}$ and $0.7 \mathrm{~V}$.

The cell is fed with high flow rates of 1.42 SLPM (standard litres per minute) for the cathode and 0.55 SLPM for the anode (equal stoichiometry ratios) in the normal state to guarantee that the cell has enough fuel and oxygen in this situation. In the next step, the anode is fed by a mixture of hydrogen and helium with the volumetric ratio of 1 to 10 . The same process is used for studying the cathode starvation; i.e. the cathode is fed by a mixture of $2 \%$ oxygen and $98 \%$ nitrogen. This decreases the stoichiometry to one tenth in both cases of the anode and cathode starvations while the gas flow rates and velocity distributions in the channels and the gas diffusion layers, and also the water distribution and the water transfer rates remain unchanged. The polarization curves for the normal and starved cases are then compared. The (partial) starvation is approved if the differences between the polarization curves are recognizable. Then, the Nyquist plots are compared.

It has to be mentioned that the difference between the heat capacities of hydrogen and helium may influence the temperature distribution inside the cell and hence the results. However, this effect does not seem to be considerable as the sensitivity analysis (see Chapter 5) revealed that even temperature changes as much as $3{ }^{\circ} \mathrm{C}$ do not change the measured Nyquist plots statistically. As the difference between the heat capacities of oxygen and nitrogen is considerably smaller, this effect is even less important in the cathode.

\subsection{Theory}

As discussed before in Chapters 3 and 4, the model shows that the impedance characteristics of a PEM fuel cell is related to the oxygen concentration in the cathode catalyst layer which means that the cathode starvation changes the cell's Nyquist plot. However, it predicts that the impedance characteristics of the cell are not affected by the hydrogen concentration in the anode catalyst layer. In other words, the anode starvation does not affect the cell's impedance. These impedances are measured here and compared against the predicted Nyquist plots to verify the model predictions.

\subsection{Results and discussions}

The measured polarization curves of the cell fed with the normal gasses and also the mixtures in the anode and cathode (described in Section 6.2) are shown in Figure 6.1. The figure shows that 
the decrease in the concentration of the reactants affects the polarization curve for both anode and cathode cases. The decrease in the oxygen concentration is more effective than the decrease in the hydrogen concentration in the anode. This could be the result of the slower cathode reaction compared to that of the anode. However, the polarization curve obtained for the starved anode also shows clearly that the partial starvation has influenced the cell performance which is distinguishable from the curve.

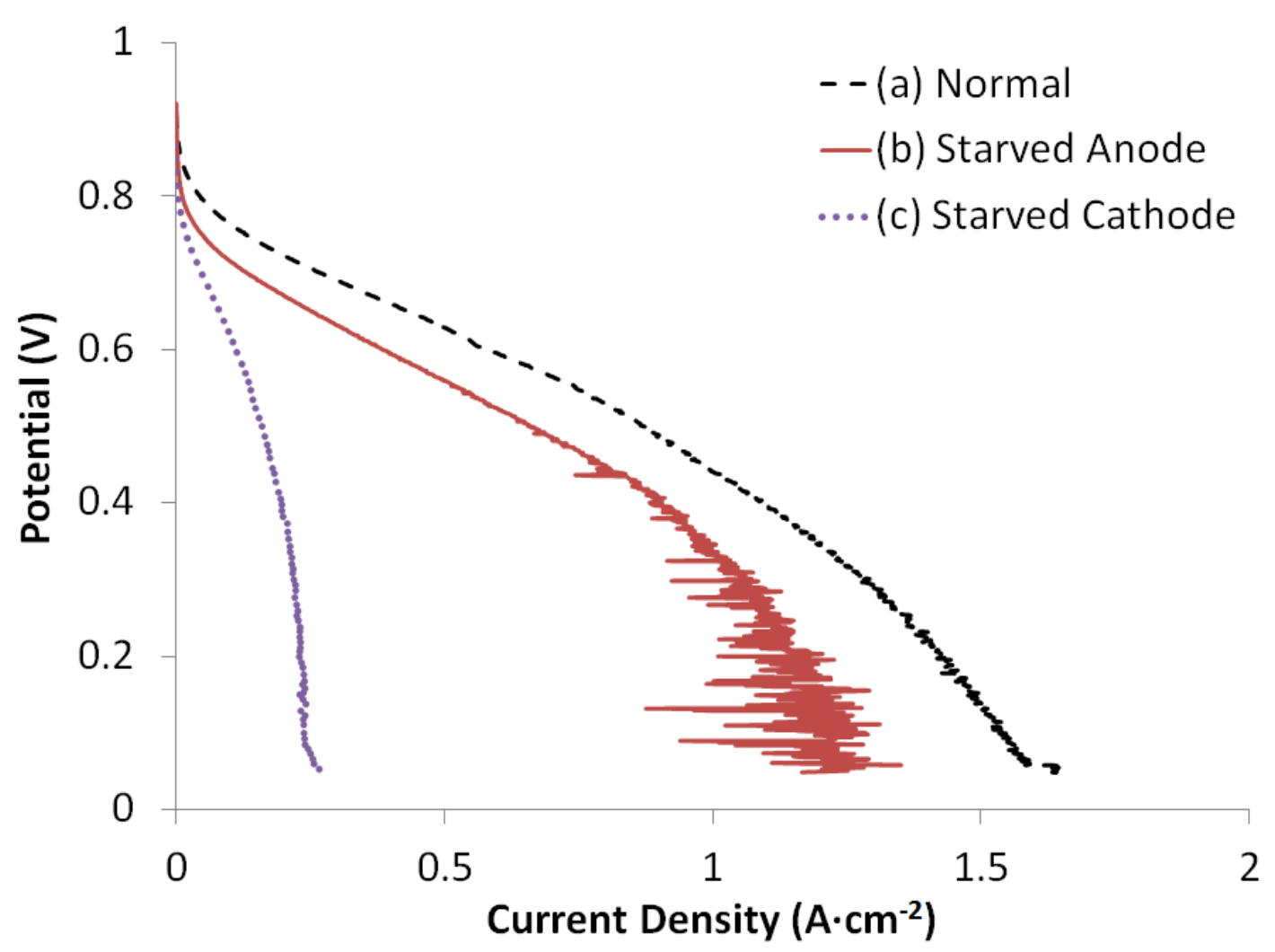

Figure 6.1 The measured polarization curves of the cell fed with (a) the normal hydrogen and air stoichiometry ratio, (b) the mixture of $10 \%$ hydrogen and $90 \%$ helium in the anode and normal air in the cathode, and (c) the normal hydrogen stoichiometry ratio in the anode and the mixture of $2 \%$ oxygen and $98 \%$ nitrogen in the cathode.

The impedance of the cell is then measured in all these cases in the potentials of $0.7 \mathrm{~V}$ and 0.75 V. Figure 6.2 depicts the measured Nyquist plots for the normal case. The model predictions are also shown in the graph. Similar to Chapter 4 , the potential of $0.7 \mathrm{~V}\left(1.92 \mathrm{~A} \cdot \mathrm{cm}^{-2}\right)$ is considered as the reference point and the model parameters are determined accordingly. The same 
parameters are then used for all the other cases (e.g. $0.75 \mathrm{~V}\left(1.01 \mathrm{~A} \cdot \mathrm{cm}^{-2}\right)$ in this figure). As it is shown, the model is in excellent agreement with the measured impedances.

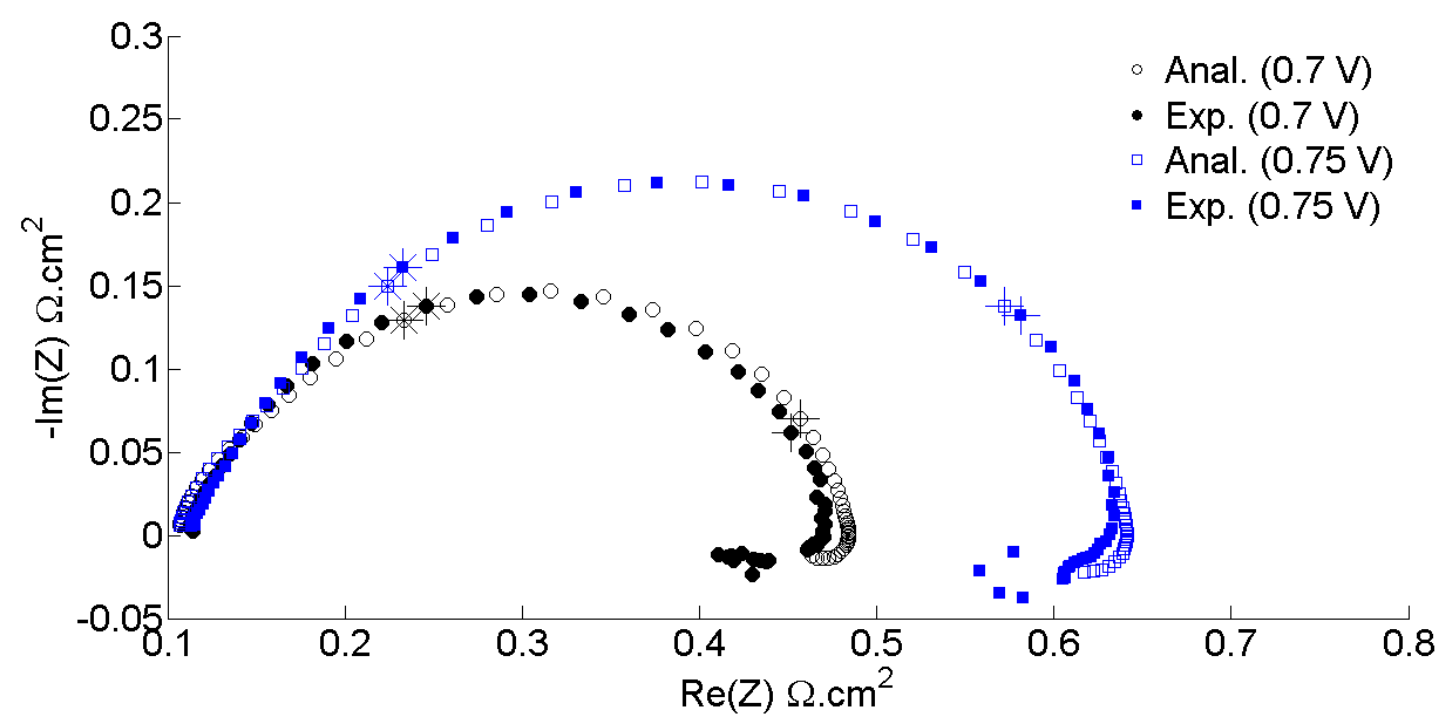

Figure 6.2 The measured and modeled Nyquist plots in the normal case. The points corresponding to the frequencies of $10 \mathrm{~Hz}$ and $100 \mathrm{~Hz}$, are marked by '+' and '* ', respectively.

The impedances are then measured for the starvation cases. The Nyquist plots for the starved cases and the related model predictions are shown in Figure 6.3. To calculate the model predictions for the starved anode, the same values as the normal case have been used. The consistency between the model and the measured impedances show that the anode starvation does not affect the Nyquist plot. This proves that the anode starvation cannot be captured by the Nyquist plots as predicted by the model. However, the cathode starvation can be clearly distinguished from the MF arc. The consistency between the measured Nyquist plots for the cathode starvation case and the model cannot be established unless the oxygen concentration in the cathode catalyst layer changes accordingly. However, when this parameter is modified to capture the measured Nyquist plot for $0.7 \mathrm{~V}\left(0.32 \mathrm{~A} \cdot \mathrm{cm}^{-2}\right)$, the same value is used for $0.75 \mathrm{~V}$ $\left(0.16 \mathrm{~A} \cdot \mathrm{cm}^{-2}\right)$, and the model predictions and the measured impedance are still in great agreement. 


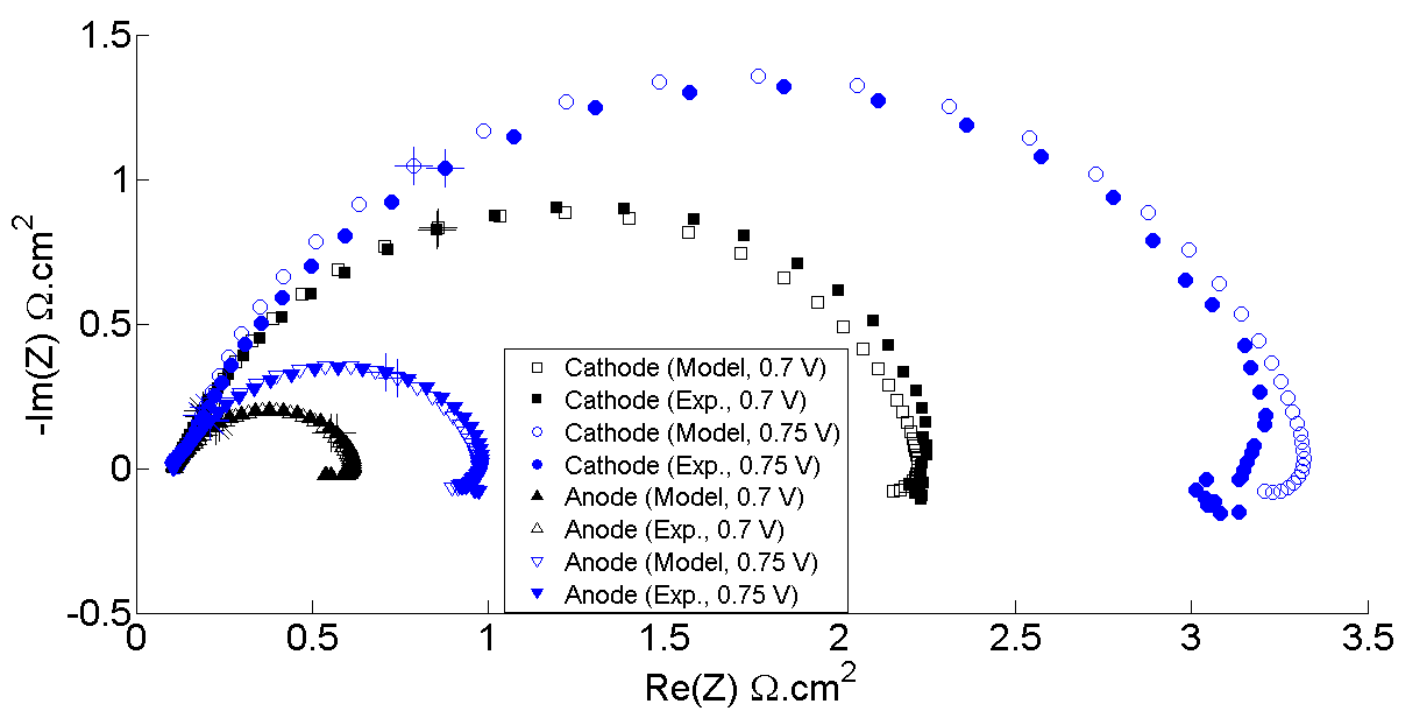

Figure 6.3 The measured and modeled Nyquist plots in the starvation of the anode (the mixture of 10\% hydrogen and $90 \%$ helium in the anode and the normal air stoichiometry ratio in the cathode) and the cathode (the normal hydrogen stoichiometry ratio in the anode and the mixture of $2 \%$ oxygen and $98 \%$ nitrogen in the cathode). The points corresponding to the frequencies of $10 \mathrm{~Hz}$ and $100 \mathrm{~Hz}$, are marked by

$$
\text { ' }+ \text { ' and ' *', respectively. }
$$

Finally, the impedances in the normal and starvation cases are compared in Figure 6.4. The figure shows that while the plot for the normal and anode starvation cases are completely similar, the cathode starvation case has a significant larger MF arc. It has to be mentioned that the current density of the measured impedance in the starved cathode case is different which affects the comparison of the Nyquist plots. To rectify this issue, the impedance of the cathode starvation case is calculated in the same current density as the normal case (i.e., $1.01 \mathrm{~A} \cdot \mathrm{cm}^{-2}$ ) using the model presented in Chapter 4, which was verified in Figures 6.2 and 6.3. This Nyquist plot is also presented in Figure 6.4. While the modification based on the current density shrinks the Nyquist plot obtained for the starved cathode case, it is still considerably larger than the normal case. In other words, cathode starvation can be detected from the MF arc inflation. However, anode starvation does not influence the impedance characteristics of the cell. 


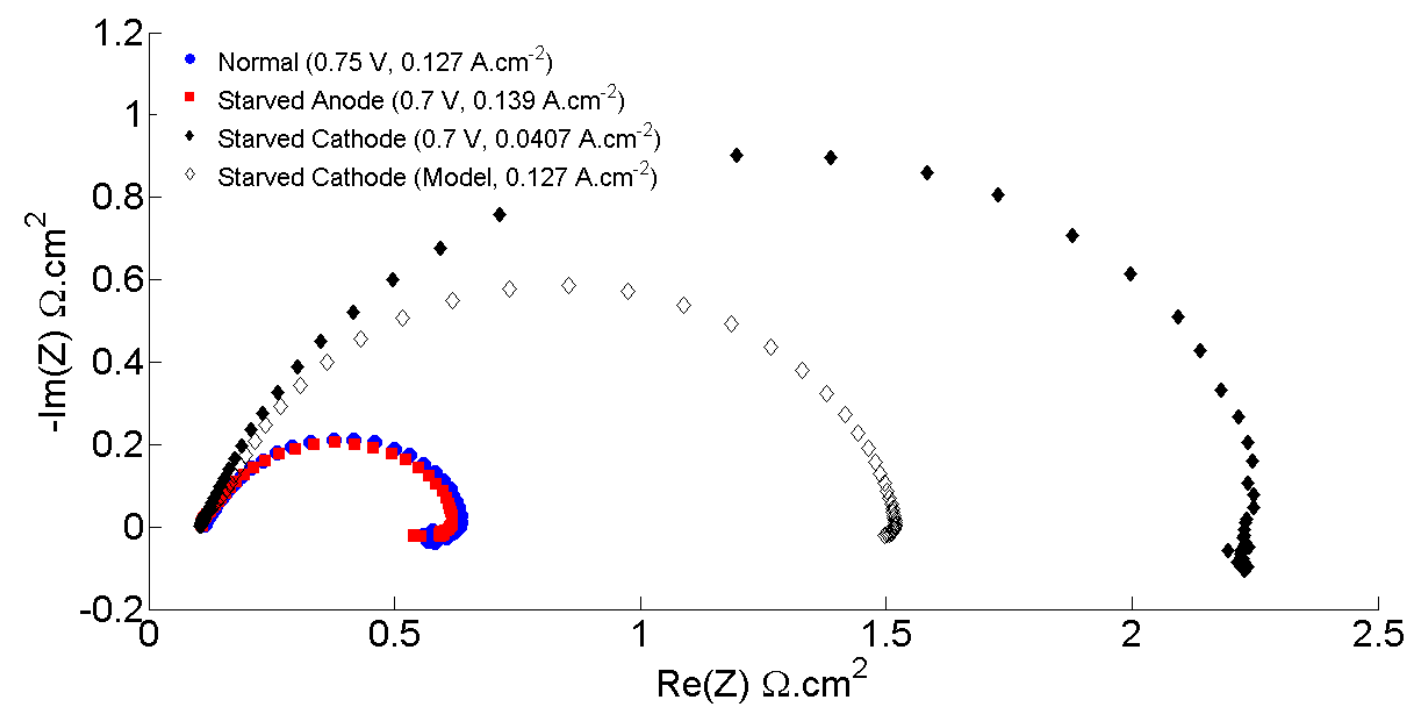

Figure 6.4 The comparison of the Nyquist plots in the normal and starved cases

It has to be mentioned that as anode starvation cannot be captured by measuring the Nyquist plot, it is reasonable to conclude that anode flooding cannot also be detected from the Nyquist plots. However, this statement can be argued as the membrane water content can increase at the flooding stage. In essence, if the excessive water in the anode GDL can influence the membrane water content, then this effect should be traced in the high frequency resistance (HFR). However, the measured impedances presented in [24, 91, 107], which were measured in the flooded conditions, do not show a considerable change in the HFR value. Thus, it is not safe to consider HFR as an indicator for anode flooding.

\subsection{Concluding remarks}

The effects of anode and cathode starvation on the impedance characteristics of the PEM fuel cell were studied. The impedance was measured while the anode was starved using a mixture of hydrogen and helium. The same process was repeated for the cathode by decreasing the percentage of the oxygen in the feeding gas. The results were also compared against the model presented in Chapter 4. It was shown that the cathode starvation can be captured from the midfrequency arc in the Nyquist plot. However, the measured Nyquist plot was not affected by anode starvation. The conclusions were in complete agreement with the model predictions. 


\section{Chapter 7. Conclusions and future work}

The main contribution of this research is the development of the first process model for studying the impedance characteristics of the proton exchange membrane (PEM) fuel cells. As discussed in Chapter 1, the EIS-based models presented in literature for PEM fuel cells are categorized into the measurement modeling and process modeling approaches. The measurement modeling approach which includes the vast majority of the published studies, is not resulted from the physical and chemical properties of the PEM fuel cells, and as a result, it can lead to debatable results. On the other hand, due to the complexity of the physical and chemical processes in the PEM fuel cell, the published models which are normally categorized as process models in literature are based on simplified assumptions which make them similar to the measurement models. While these assumptions seem to be reasonably accurate at the first sight, the discussions presented in Chapter 1 showed that these models can also lead to dubious conclusions similar to the measurement models. These models were categorized in Chapter 1 as semi-process models.

In this thesis, the first process model was presented which was not based on the abovementioned simplified assumptions. The three losses (i.e., ohmic, activation and concentration overpotentials) were separately modeled and verified against impedance measurements: the ohmic overpotential was studied and the corresponding process model was presented in Chapter 2, while the predicted impedances were compared against the measured impedances reported in literature. Chapter 3 covered the details of the process models developed for the activation and concentration overpotentials. These models were then combined in Chapter 4 and the resultant complete process model of the PEM fuel cell was compared against the impedances measured in different operating conditions. These comparisons proved that unlike the semi-process models presented in literature before, this process model is able to predict the impedance characteristics 
of the cell in a wide range of operating conditions. Two different applications of the model were presented: The sensitivity of the impedance characteristics of the PEM fuel cell was analysed using the presented model in Chapter 5. Next, anode and cathode starvations were studied in Chapter 6 and the model predictions were verified against the impedance measurements in the starved cells.

\subsection{Conclusions}

The main outcome of the model presented in this thesis was that the impedance characteristics (specifically the Nyquist plot) of the PEM fuel cell was clearly translated and related to the physical and chemical properties of the cell. The model identified (i) the parameters of the cell controlling each part of the Nyquist plot, and (ii) the data that can be extracted from the measured impedances. Also, the model clarified (i) the phenomena (or failure modes) which cannot be detected by the impedance measuring, and (ii) the properties do not affect the Nyquist plot of the cell. The conclusions can be summarised as follows:

- The high frequency resistance (HFR) shows the water content of the membrane. This parameter can be monitored to detect the membrane dehydration.

- The high frequency (HF) arc is the result of the anode impedance and does not change in wide range of operating conditions.

- The mid-frequency (MF) arc is the result of the cathode impedance. The cathode starvation can be detected by monitoring the diameter of this arc.

- Unlike cathode starvation, anode starvation cannot be detected from the Nyquist plot.

- The low frequency (LF) arc is mostly controlled by the membrane diffusivity.

- The inductive loop is controlled by water transport in the cathode gas diffusion layer (GDL). The flooding of the cathode GDL can be detected from this loop.

- The potential changes less than $3 \mathrm{mV}$, current density changes less than $5 \mathrm{~mA} \cdot \mathrm{cm}^{-2}$, temperature changes less than $3{ }^{\circ} \mathrm{C}$, and anode and cathode relative humidity changes less than $5 \%$ do not certainly affect the measured impedances.

\subsection{Proposed future work}

The future work summarized here is related to potential applications of the model. The model can be used to monitor and diagnose the PEM fuel cells during the long-run performances. As mentioned, most of the failure modes of the cell can be detected by measuring the impedance 
and using the model. This monitoring can be done separately in different cells in a fuel cell stack to evaluate the state of the health of different cells in the stack. It was reported in literature that starvation or flooding do not usually happen simultaneously in all the cells in the stack, and hence, a fast, accurate and in-situ monitoring tool can considerably improve the cell controlling systems.

The model can also be used to enhance the methodology and results obtained regarding the properties and performance of the fuel cells. As most of the physical and chemical parameters of the cell cannot be easily measured or estimated in a working cell by in-situ measurements, the studies regarding the failure modes need improvements. As an example, the dehydration tests are achieved by feeding dry gasses to the anode and cathode of the cell. However, the cell itself produces water in the reaction, and hence, it is not clear how much the membrane water content is affected by this test. Using this model, the membrane water content can be estimated and traced during the test, and the dehydration effects can be clearly monitored. Using the model, similar uncertainties associated with the starvation and flooding experiments can be rectified. Moreover, replacing a part of the cell (e.g., the cathode GDL) normally affects several phenomena in the cell (e.g., the water transport in cathode GDL, the air transport to the cathode catalyst layer, the membrane water content, and the overall ohmic resistance of the cell). While the overall result of all these effects on the performance of the cell is usually considered to evaluate the new part, the pros and cons are not determined, and hence, the future improvements towards rectifying the cons are not directed properly. However, using the model, these different effects can be separated and discussed individually. This methodology can be specifically used in the studies such as the catalyst loading, the membrane thickness, the GDL transport characteristics and also the sensitivity of the cell to the fuel impurities, which can improve the PEM fuel cell final cost and performance considerably.

Finally, it has to be mentioned that the model improvements can also be considered and recommended as future works. The assumptions used in the analytical analysis (such as having enough protons in the cathode side compared to the oxygen atoms) can be re-examined in extreme conditions. The multi-phase oxygen and water (liquid and vapour) transport in the cathode gas diffusion layer can be studied and an analytical or experimental relation can be determined for effective water diffusivity. Also, the correlation between the equivalent circuit and the polarization curve can be studied and different overpotentials can be estimated. 


\section{Bibliography}

[1] S.M. Rezaei Niya, M. Hoorfar, J. Power Sources 240 (2013) 281-293.

[2] R. O’hayre, S. Cha, W. Colella, F.B. Prinz, Fuel cell fundamentals, second ed., John Wiley \& Sons, 2009.

[3] D. Papageorgopoulos, 2011 DOE Annual Progress Report, V. Fuel cells, Fuel cells sub-program overview. http://www.hydrogen.energy.gov/annual_progress11_fuelcells.html.

[4] B.R. Friess, M. Hoorfar, Int. J. Hydrogen Energy 37 (2012) 7719-7729.

[5] A. Bazylak, Liquid water transport in fuel cell gas diffusion layers, PhD thesis, University of Victoria, 2008.

[6] A. Bazylak, Int. J. Hydrogen Energy 34 (2009) 3845-3857.

[7] J. Wu, X.Z. Yuan, H. Wang, M. Blanco, J.J. Martin, J. Zhang, Int. J. Hydrogen Energy 33 (2008) 1735-1746.

[8] X. Yuan, H. Wang, J.C. Sun , J. Zhang, Int. J. Hydrogen Energy 32 (2007) 4365-4380.

[9] P. Agarwal, M.E. Orazem, L.H. Garcia-Rubio, J. Electrochem. Soc. 139 (1992) 1917-1927.

[10] G.J. Brug, A.L.G. Van Den Eeden, M. Sluyters-Rehbach, J.H. Sluyters, J. Electroanal. Chem. 176 (1984) 275-295.

[11] S.M. Rezaei Niya, M. Hejabi, F. Gobal, J. Power Sources 195 (2010) 5789-5793.

[12] P. Gode, F. Jaouen, G. Lindbergh, A. Lundbald, G. Sundholm, Electrochimica Acta 48 (2003) 4175-4187.

[13] F. Jaouen, G. Lindbergh, K. Wiezell, J. Electrochem. Soc. 150 (2003) A1711-A1717.

[14] X. Yuan, J.C. Sun, H. Wang, J. Zhang, J. Power Sources 161 (2006) 929-937.

[15] N.V. Dale, M.D. Mann, H. Salehfar, A.M. Dhride, T. Han, J. Fuel Cell Sci. Technol. 7 (2010) 031010 .

[16] S.J. Andreasen, J.L. Jespersen, E. Schaltz, S.K. Kaer, Fuel Cells 9 (2009) 463-473.

[17] S.J. Andreasen, J.R. Vang, S.K. Kaer, Int. J. Hydrogen Energy 36 (2011) 9815-9830.

[18] T. Soboleva, X. Xie, Z. Shi, E. Tsang, T. Navessin, S. Holdcroft, J. Electroanal. Chem. 622 (2008) 145-152.

[19] P. Agarwal, M.E. Orazem, J. Electrochem. Soc. 142 (1995) 4159-4168.

[20] M.A. Danzer, E.P. Hofer, J. Power Sources 190 (2009) 25-33. 
[21] J.E.B. Randles, Discuss. Faraday Soc. 1 (1947) 11-19.

[22] J.E.B. Randles, K.W. Somerton, Trans. Faraday Soc. 48 (1952) 937-950.

[23] D.C. Grahame, J. Electrochem. Soc. 99 (1952) 370C-385C.

[24] N. Fouquet, C. Doulet, C. Nouillant, G. Dauphin-Tanguy, B. Ould-Bouamama, J. Power Sources 159 (2006) 905-913.

[25] M. Cimenti, D. Bessarabov, M. Tam, J. Stumper, ECS Trans. 28 (2010) 147-157.

[26] D. Malevich, J.G. Pharoah, B.A. Peppley, K. Karan, ECS Trans. 41 (2011) 721-732.

[27] J.H. Jang, S. Jeon, J.H. Cho, S. Kim, S. Lee, E. Cho, H. Kim, J. Han, T. Lim, J. Electrochem. Soc. 156 (2009) B1293-B1300.

[28] A.P. Young, J. Stumper, E. Gyenge, J. Electrochem. Soc. 156 (2009) B913-B922.

[29] Y. Liu, M.W. Murphy, D.R. Baker, W. Gu, C. Ji, J. Jorne, H.A. Gasteiger, J. Electrochem. Soc. 156 (2009) B970-B980.

[30] D. Malevich, B.R. Jayasankar, E. Halliop, J.G. Phaorah, B.A. Peppley, K. Karan, J. Electrochem. Soc. 159 (2012) F888-F895.

[31] R. de Levie, Advances in electrochemistry and electrochemical engineering, John Wiley \& Sons, 1967.

[32] R. Makharia, M.F. Mathias, D.R. Baker, J. Electrochem. Soc. 152 (2005) A970-A977.

[33] D. Aaron, S. Yiacoumi, C. Tsouris, Separation Sci. Tech. 43 (2008) 2307-2320.

[34] D. Malevich, E. Halliop, B.A. Peppley, J.G. Pharoah, K. Karan, ECS Trans. 16 (2008) 17631774.

[35] D. Malevich, E. Halliop, M.S. Saha, J. Suryana, B.A. Peppley, J.G. Pharoah, K. Karan, 216 ${ }^{\text {th }}$ ECS Meet. MA2009-02, October 4-9, Vienna, Austria.

[36] C. Brunetto, A. Moschetto, G. Tina, Electr. Power Sys. Res. 79 (2009) 17-26.

[37] J. Hou, W. Song, H. Yu, Y. Fu, L. Hao, Z. Shao, B. Yi, J. Power Source 176 (2008) 118-121.

[38] J. Hou, H. Yu, M. Yang, W. Song, Z. Shao, B. Yi, Int. J. Hydrogen Energy 36 (2011) I2444I2451.

[39] A. Pistono, C.A. Rice-York, V. Boovaragavan, J. Electrochem. Soc. 158 (2011) B233-B238

[40] R. Mukundan, J.R. Davey, R.W. Lujan, J. Spendelow, Y.S. Kim, D.S. Hussey, D.L. Jacobson, M. Arif, R.L. Borup, ECS Trans. 16 (2008) 1939-1950.

[41] I. Nitta, O. Himanen, M. Mikkola, Electrochem. Commun. 10 (2008) 47-51.

[42] J.I. Eastcott, E.B. Easton, Electrochimica Acta 54 (2009) 3460-3466.

[43] J.I. Eastcott, K.M. Yarrow, A.W. Pedersen, E.B. Easton, J. Power Sources 197 (2012) 102-106.

[44] S.J. Seo, J.J. Woo, S.H. Yun, H.J. Lee, J.S. Park, T. Xu, T.H. Yang, J. Lee, S.H. Moon, Phys. Chem . Chem. Phys. 12 (2010) 15291-15300.

[45] S.C. Manzo, P. Rama, R. Chen, J. Electrochem. Soc. 157 (2010) B1865-B1871.

[46] D. Malevich, E. Halliop, B.A. Peppley, J.G. Pharoah, K. Karan, J. Electrochem. Soc. 156 (2009) B216-B224.

[47] S.C. Manzo, P. Rama, R. Chen, J. Electrochem. Soc. 157 (2010) B400-B408.

[48] A.A. Kulikovsky, M. Eikerling, J. Electroanal. Chem. 691 (2013) 13-17.

[49] Z. Tang, D.H.C. Chua, J. Electrochem. Soc. 157 (2010) B868-B873. 
[50] T. Kim, S. Yim, Y. Choi, T. Yang, Y. Yoon, S. Park, C. Kim, I. Sung, Electrochem. Commun. 13 (2011) 1313-1316.

[51] P. Mu, W. Qingyu, Int. J. Energy Res. 35 (2011) 923-928.

[52] S. Park, Y. Shao, H. Wan, V.V. Viswanathan, S.A. Towne, P.C. Rieke, J. Liu, Y. Wang, J. Phys. Chem. C 115 (2011) 22633-22639.

[53] S. Park, Y. Shao, R. Kou, V.V. Viswanathan, S.A. Towne, P.C. Rieke, J. Liu, Y. Lin, Y. Wang, J. Electrochem. Soc. 158 (2011) B297-B302.

[54] S. Park, Y. Shao, H. Wan, P.C. Rieke, V.V. Viswanathan, S.A. Towne, L.V. Saraf, J. Liu, Y. Lin, Y. Wang, Electrochem. Commun. 13 (2011) 258-261.

[55] T. Wang, J. He, D. Sun, J. Zhou, Y. Guo, X. Ding, S. Wu, J. Zhao, J. Tang, Corros. Sci. 53 (2011) 1498-1504.

[56] Q. Zheng, X. Cheng, T. Jao, F. Weng, A. Su, Y. Chiang, J. Power Sources 201 (2012) 151-158.

[57] J. Zhang, L. Zhang, C.W.B. Bezerra, H. Li, Z. Xia, J. Zhang, A.L.B. Marques, E.P. Marques, Electrochimica Acta 54 (2009) 1737-1743.

[58] V.C. Velan, G. Velayutham, N. Hebalkar, K.S. Dhathathreyan, Int. J. Hydrogen Energy 36 (2011) 14815-14822.

[59] Y. Bultel, K. Wiezell, F. Jaouen, P. Ozil, G. Lindbergh, Electrochimica Acta 51 (2005) 474-488.

[60] M. Maidhily, N. Rajalakshmi, K.S. Dhathathreyan, Int. J. Hydrogen Energy 36 (2011) 1235212360.

[61] G. Dotelli, L. Omati, P.G. Stampino, P. Grassini, D. Brivio, J. Power Sources 196 (2011) 89558966.

[62] L. Omati, P.G. Stampino, G. Dotelli, D. Brivio, P. Grassini, Int. J. Hydrogen Energy 36 (2011) 8053-8062.

[63] P.G. Stampino, L. Omati, C. Cristiani, G. Dotelli, Fuel Cells 10 (2010) 270-277.

[64] J.H. Jang, S. Jeon, J.H. Cho, S.-K. Kim, S.-Y. Lee, E.A. Cho, H.-J. Kim, J. Han, T.-H. Lim, J. Electrochem. Soc. 156 (2009) B1293-B1300.

[65] M.C. Lefebvre, R.B. Martin, P.G. Pickup, Electrochem. Solid State Lett. 2 (1999) 259-261.

[66] H. Chang, C. Lin, M. Chang, H. Shiu, W. Chang, F. Tsau, J. Power Sources 196 (2011) 37733780 .

[67] K. Nakagawa, Y. Yasumura, N. Thongprachan, N. Sano, Chem. Eng. Process. 50 (2011) 22-30.

[68] S. Park, B.N. Popov, Fuel 90 (2011) 436-440.

[69] R.P. Ramasamy, E.C. Kumbur, M.M. Mench, W. Liu, D. Moore, M. Murthy, Int. J. Hydrogen Energy 33 (2008) 3351-3367.

[70] S.D. Mikhailenko, M.D. Guiver, S. Kaliaguine, Solid State Ion. 179 (2008) 619-624.

[71] C.H. Lee, H.B. Park, Y.M. Lee, R.D. Lee, Ind. Eng. Chem. Res. 44 (2005) 7617-7626.

[72] P.G. Escribano, C.D. Rio, J.L. Acosta, J. Power Sources 187 (2009) 98-102.

[73] Y. Liu, C. Ji, W. Gu, J. Jorne, H.A. Gasteiger, J. Electrochem. Soc. 158 (2011) B614-B621.

[74] M. Eikerling, A.A. Kornyshev, J. Electroanal. Chem. 475 (1999) 107-123.

[75] B. Legros, P.X. Thivel, Y. Bultel, M. Boinet, R.P. Nogueira, Electrochem. Solid-state Lett. 12 (2009) B116-B118.

[76] A. Huth, B. Schaar, T. Oekermann, Electrochimica Acta 54 (2009) 2774-2780. 
[77] A. Navarro, C.D. Rio, J.L. Acosta, Solid State Ion. 180 (2009) 1505-1510.

[78] M.N. Tsampas, C.G. Vayenas, $217^{\text {th }}$ ECS Meeting, 2010, Vancouver, Canada.

[79] M.N. Tsampas, S. Brosda, C.G. Vayenas, Electrochimica Acta 56 (2011) 10582-10592.

[80] M. Keddam, J.-F. Lizee, C. Pallotta, H. Takenouti, J. Electrochem. Soc. 131 (1984) 2016-2024.

[81] I. Epelboin, M. Keddam, Electrochimica Acta 17 (1972) 177-186.

[82] X. Wang, J.-M. Hu, I.-M. Hsing, J. Electroanal. Chem. 562 (2004) 73-80.

[83] C. Lamy, D.J. Jones, C. Coutanceau, P. Brault, S. Martemianov, Y. Bultel, Electrochimica Acta 56 (2011) 10406-10423.

[84] N. Wagner, T. Kaz, K.A. Friedrich, Electrochimica Acta 53 (2008) 7475-7482.

[85] D.C. Martinez-Casillas, G. Vazquez-Huerta, J.F. Perez-Robles, O. Solorza-Feria, J. Power Sources 196 (2011) 4468-4474.

[86] S.S. Pethaiah, G.P. Kalaignan, M. Ulaganathan, J. Arunkumar, Ionics 17 (2011) 361-366.

[87] H. Li, J. Zhang, K. Fatih, Z. Wang, Y. Tang, Z. Shi, S. Wu, D. Song, J. Zhang, N. Jia, S. Wessel, R. Abouatallah, N. Joos, J. Power Sources 185 (2008) 272-279.

[88] H. Li, H. Wang, W. Qian, S. Zhang, S. Wessel, T.T.H. Cheng, J. Shen, S. Wu, J. Power Sources 196 (2011) 6249-6255.

[89] M.J. Martinez-Rodriguez, E.B. Fox, W.D. Rhodes, C.S. McWhorter, S. Greenway, H.R. ColonMercado, J. Electrochem. Soc. 158 (2011) B698-B702.

[90] H. Nakajima, T. Konomi, T. Kitahara, H. Tachibana, J. Fuel Cell Sci. Tech. 5 (2008) 041013-1-6.

[91] T. Kadyk, R. Hanke-Rauschenbach, K. Sundmacher, J. Electroanal. Chem. 630 (2009) 19-27.

[92] T. Kadyk, R. Hanke-Rauschenbach, K. Sundmacher, J. Appl. Electrochem. 41 (2011) 1021-1032.

[93] R. Lin, C. Cao, H. Zhang, H. Huang, J. Ma, Int. J. Hydrogen Energy 37 (2012) 4648-4656.

[94] S.K. Roy, H. Hagelin-Weaver, M.E. Orazem, J. Power Sources 196 (2011) 3736-3742.

[95] H. Nara, S. Tominaka, T. Momma, T. Osaka, J. Electrochem. Soc. 158 (2011) B1184-B1191.

[96] F. Xu, L. Zhang, X. Cheng, Y. Zhang, 214 ${ }^{\text {th }}$ ECS Meet., 2008, Honolulu, HI.

[97] T.A. Aarhaug, A.M. Svensson, A. Odegard, S. Moller-Holst, 215 ${ }^{\text {th }}$ ECS Meet., MA2009-01, 2009, San Francisco.

[98] F. Sergi, G. Brunaccini, A. Stassi, A. Di Blasi, G. Dispenza, A.S. Arico, M. Ferraro, V. Antonucci, Int. J. Hydrogen Energy 36 (2011) 10908-10916.

[99] D. Seo, J. Lee, S. Park, J. Rhee, S.W. Choi, Y. Shul, Int. J. Hydrogen Energy 36 (2011) 18281836.

[100] Y. Cho, T. Jeon, J.W. Lim, Y. Cho, M. Ahnb, N. Jung, S. Yoo, W. Yoon, Y. Sung, Int. J. Hydrogen Energy 36 (2011) 4394-4399.

[101] Y. Cho, S. Kim, J.W. Lim, Y.S. Kang, Y. Cho, O. Kim, N. Kwon, O.J. Kwon, W. Yoon, H. Choe, Y. Sung, Int. J. Hydrogen Energy 37 (2012) 2490-2497.

[102] M. Bautista, Y. Bultel, P. Ozil, Chem. Eng. Res. Des. 82 (2004) 907-917.

[103] T. Kurz, A. Hakenjos, J. Kramer, M. Zedda, C. Agert, J. Power Sources, 180 (2008) 742-747.

[104] S.K. Roy, M.E. Orazem, J. Power Sources 184 (2008) 212-219.

[105] S.K. Roy, M.E. Orazem, J. Electrochem. Soc. 156 (2009) B203-B209. 
[106] I.A. Schneider, M.H Bayer, A. Wokaun, G.G. Scherer, J. Electrochem. Soc. 155 (2008) B783B792.

[107] S. Rodat, S. Sailler, F. Druart, P.-X. Thivel, Y. Bultel, P. Ozil, J. Appl. Electrochem. 40 (2010) 911-920.

[108] O.J. Kwon, M.S. Kang, S.H. Ahn, I. Choi, K.U. Lee, J.H. Jeong, I. Han, J.C. Yang, J.J. Kim, Int. J. Hydrogen Energy 36 (2011) 9799-9804.

[109] J. Mainka, G. Maranzana, J. Dillet, S. Didierjean, O. Lottin, FDFC08 2008 Nancy, France.

[110] J. Mainka, G. Maranzana, J. Dillet, S. Didierjean, O. Lottin, J. Electrochem. Soc. 157 (2010) B1561-1568.

[111] J. Mainka, G. Maranzana, J. Dillet, S. Didierjean, O. Lottin, WHEC-2010, 2010, May 16-21, Essen.

[112] S. Asghari, A. Mokmeli, M. Samavati, Int. J. Hydrogen Energy 35 (2010) 9283-9290.

[113] M. Cimenti, M. Tam, J. Stumper, Electrochem. Solid-state Lett. 12 (2009) B131-B134.

[114] A.M. Dhirde, N.V. Dale, H. Salehfar, M.D. Mann, T.H. Han, IEEE Trans. Energy Convers. 25 (2010) 778-786.

[115] M.A. Travassos, V.V. Lopes, R.A. Silva, A.Q. Novais, C.M. Rangel, Int. J. Hydrogen Energy 18 (2013) 7684-7696.

[116] X. Yuan, J.C. Sun, H. Wang, H. Li, J. Power Sources 205 (2012) 340-344.

[117] T.E. Springer, T.A. Zawodzinski, M.S. Wilson, S. Gottesfeld, J. Electrochem. Soc. 143 (1996) 587-599.

[118] M.U. Iftikhar, D. Riu, F. Drurat, S. Rosini, Y. Bultel, N. Retiere, J. Power Sources 160 (2006) 1170-1182.

[119] J. Deseure, J. Power Sources 178 (2008) 323-333.

[120] M.E. Orazem, ECS Trans. 50 (2012) 247-260.

[121] A.K. Meland, S. Kjelstrup, J. Electroanal. Chem. 610 (2007) 171-178.

[122] D. Kramer, I.A. Schneider, A. Wokaun, G.G. Scherer, ECS Trans. 3 (2006) 1249-1258.

[123] G. Maranzana, J. Mainka, O. Lottin, J. Dillet, A. Lamibrac, A. Thomas, S. Didierjean, Electrochim. Acta 83 (2012) 13-27.

[124] J. Mainka, G. Maranzana, J. Dillet, S. Didierjean, O. Lottin, J. Power Sources 253 (2014) 381391.

[125] B.A. Boukamp, Solid State Ionics 62 (1993) 131-141.

[126] M.E. Orazem, B. Tribollet, Electrochim. Acta 53 (2008) 7360-7366.

[127] C. Wang, J. Electrochem. Soc. 145 (1998) 1801-1812.

[128] S.M. Rezaei Niya, M. Hoorfar, Electrochim. Acta 120 (2014) 193-203.

[129] R. O’hayre, D.M. Barnett, F.B. Prinz, J. Electrochem. Soc. 152 (2005) A439-A444.

[130] T.E. Springer, T.A. Zawodzinski, S. Gottesfeld, J. Electrochem. Soc. 138 (1991) 2334-2342.

[131] N. Zamel, X. Li, Int. J. Energy Res. 32 (2008) 698-721.

[132] W. He, J.S. Yi, T.V. Nguyen, AIChe J. 46 (2000) 2053-2064.

[133] C.Y. Wang, P. Cheng, Adv. Heat Transfer 30 (1997) 93-196.

[134] T.S. Sorensen, S. Kjelstrup, J. Colloid Interf. Sci. 248 (2002) 355-375. 
[135] J.D. Jackson, Classical Electrodynamics, John Wiley \& Sons, 1962.

[136] S.M. Rezaei Niya, M. Hoorfar, ASME ESFuelCell2014 (2014), Boston, USA.

[137] S.M. Rezaei Niya, M. Hoorfar, CSME International Congress (2014), Toronto, Canada.

[138] W. Sheng, H.A. Gasteiger, Y. Shao-Horn, J. Electrochem. Soc. 157 (2010) B1529-B1536.

[139] C.G. Zoski, J. Phys. Chem. B 107 (2003) 6401-6405.

[140] R.M.Q. Mello, E.A. Ticianelli, Electrochem. Acta 42 (1997) 1031-1039.

[141] C.Y. Jung, W.J. Kim, S.C. Yi, J. Membrane Sci. 341 (2009) 5-10.

[142] http://hydrogen.pnl.gov/cocoon/morf/hydrogen/article/701

[143] J.H. Nam, M. Kaviany, Int. J. Heat Mass Tran. 46 (2003) 4595-4611.

[144] N. Zamel, N.G.C. Astrath, X. Li, J. Shen, J. Zhou, F.B.G. Astrath, H. Wang, Z.S. Liu, Chem. Eng. Sci. 65 (2010) 931-937.

[145] J. Maruyama, M. Inaba, K. Katakura, Z. Ogumi, Z. Takehara, J. Electroanal. Chem. 447 (1998) 201-209.

[146] Y. Sun, J. Lu, L. Zhuang, Electrochim. Acta 55 (2010) 844-850.

[147] N.M. Markovic, B.N. Grgur, P.N. Ross, J. Phys. Chem. B 101 (1997) 5405-5413.

[148] T.J. Schmidt, P.N. Ross, N.M. Markovic, J. Electroanal. Chem. 524 (2002) 252-260.

[149] H.A. Gasteiger, J.E. Panels, S.G. Yan, J. Power Sources 127 (2004) 162-171.

[150] K.C. Neyerlin, W. Gu, J. Jorne, H.A. Gasteiger, J. Electrochem. Soc. 154 (2007) B631-B635.

[151] J. Jiang, A. Kucernak, J. Electroanal. Chem. 567 (2004) 123-137.

[152] K. Seto, A. Iannelli, B. Love, J. Lipkowski, J. Electroanal. Chem. 226 (1987) 351-360.

[153] J.X. Wang, S.R. Brankovic, Y. Zhu, J.C. Hanson, R.R. Adzic, J. Electrochim. Soc. 150 (2003) A1108-A1117.

[154] M.J. Croissant, T. Napporn, J.-M. Leger, C. Lamy, Electrochim. Acta 43 (1998) 2447-2457.

[155] E. Barsoukov, J.R. Macdonald, Impedance Spectroscopy: Theory, Experiment, and Applications, Second ed., John Wiley \& Sons, 2005.

[156] T. Pajkossy, D.M. Kolb, Electrochim. Acta 46 (2001) 3063-3071.

[157] M. Rosen, D.R. Flinn, S. Schuldiner, J. Electrochem. Soc. 116 (1969) 1112-1116.

[158] S. Schuldiner, R.M. Roe, J. Electrochem. Soc. 110 (1963) 332-338.

[159] B.D. Cahan, J.S. Wainright, J. Electrochem. Soc. 140 (1993) L185-L186.

[160] H.A. Gasteiger, M.F. Mathias, Proceed. Proton-Conducting Membrane Fuel Cells III Symposium (2002).

[161] T. Kadyk, S. Kirsch, R. Hanke-Rauschenbach, K. Sundmacher, Electrochim. Acta 56 (2011) 10593-10602.

[162] P.H. Bottelberghs, G.H.J. Broers, J. Electroanal. Chem. 67 (1976) 155-167.

[163] L. Nyikos, T. Pajkossy, Electrochim. Acta 30 (1985) 1533-1540.

[164] U. Rammelt, G. Reinhard, Electrochim. Acta 35 (1990) 1045-1049.

[165] B. Hirschorn, M. Orazem, B. Tribollet, V. Vivier, I. Frateur, M. Musiani, J. Electrochem. Soc. 157 (2010) C458-C463. 
[166] B. Hirschorn, M. Orazem, B. Tribollet, V. Vivier, I. Frateur, M. Musiani, J. Electrochem. Soc. 157 (2010) C452-C457.

[167] B. Hirschorn, M. Orazem, B. Tribollet, V. Vivier, I. Frateur, M. Musiani, Electrochim. Acta 55 (2010) 6218-6227.

[168] A. Parthasarathy, S. Srinivasan, A.J. Appleby, C.R. Martin, J. Electrochem. Soc. 139 (1992) 2530-2537.

[169] S. Mukerjee, S. Srinivasan, M.P. Soriaga, J. McBreen, J. Phys. Chem. 99 (1995) 4577-4589.

[170] A.J. Appleby, Electroanal. Chem. 24 (1970) 97-117.

[171] P.D. Beattie, V.I. Basura, S. Holdcroft, J. Electroanal. Chem. 468 (1999) 180-192.

[172] B.T. Huang, Y. Chatillon, C. Bonnet, F. Lapicque, S. Leclerc, M. Hinaje, S. Rael, Fuel Cells 12 (2012) 335-346.

[173] G. Maranzana, C. Moyne, J. Dillet, S. Didierjean, O. Lottin, J. Power Sources 195 (2010) 59905995.

[174] A. Damjanovic, J. Electroanal. Chem. 355 (1993) 57-77.

[175] E. Yeager, Electrochim. Acta 29 (1984) 1527-1537.

[176] T. Kim, S. Lee, H. Park, Int. J. Hydrogen Energy 35 (2010) 8631-8643.

[177] W. Sheng, M. Myint, J.G. Chen, Y. Yan, Energy Environ. Sci. 6 (2013) 1509-1512.

[178] D.W. Banham, J.N. Soderberg, V.I. Birss, J. Phys. Chem. C 113 (2009) 10103-10111.

[179] P.D. Das, X. Li, Z. Liu, J. Electroanal. Chem. 604 (2007) 72-90.

[180] A. Rowe, X. Li, J. Power Sources 102 (2001) 82-96.

[181] C. Marr, X. Li, J. Power Sources 77 (1999) 17-27.

[182] K.-M. Yin, C.-P. Chang, Fuel Cells 11 (2011) 888-896.

[183] J. Le Canut, R. Latham, W. Merida, D.A. Harrington, J. Power Sources 192 (2009) 457-466.

[184] A. Iranzo, M. Munoz, F.J. Pino, F. Rosa, J. Power Sources 196 (2011) 4264-4269.

[185] J. Lee, J. Lee, W. Choi, K. Park, H. Sun, J. Oh, J. Power Sources 195 (2010) 6001-6007.

[186] M. Mamlouk, K. Scott, Electrochim. Acta 56 (2011) 5493-5512.

[187] M. Perez-Page, V. Perez-Herranz, Int. J. Hydrogen Energy 39 (2014) 4009-4015.

[188] Y. Tang, J. Zhang, C. Song, H. Liu, J. Zhang, H. Wang, S. Mackinnon, T. Peckham, J. Li, S. McDermid, P. Kozak, J. Electrochem. Soc. 153 (2006) A2036-A2043.

[189] X. Yan, M. Hou, L. Sun, D. Liang, Q. Shen, H. Xu, P. Ming, B. Yi, Int. J. Hydrogen Energy 32 (2007) 4358-4364.

[190] J. O’Rourke, M. Ramani, M. Arcak, Int. J. Hydrogen Energy 33 (2008) 4694-4701.

[191] R. Hanke-Rauschenbach, M. Mangold, K. Sundmacher, Rev. Chem. Eng. 27 (2011) 23-52.

[192] S.K. Roy, M.E. Orazem, ECS Trans. 13 (2008) 153-169.

[193] S.K. Roy, M.E. Orazem, J. Electrochem. Soc. 154 (2007) B883-B891.

[194] D. Gerteisen, A. Hakenjos, J.O. Schumacher, J. Power Sources 173 (2007) 346-356.

[195] W.Q. Tao, C.H. Min, X.L. Liu, Y.L. He, B.H. Yin, W. Jiang, J. Power Sources 160 (2006) 359373. 
[196] C.H. Min, Y.L. He, X.L. Liu, B.H. Yin, W. Jiang, W.Q. Tao, J. Power Sources 160 (2006) 374385.

[197] J.M. Correa, F.A. Farret, V.A. Popov, M.G. Simões, IEEE T. Energy Conver. 20 (2005) 211-218.

[198] S.M. Rezaei Niya, M. Hoorfar, ASME ESFUELCELL2012 (2012) 345-349.

[199] S.M. Rezaei Niya, P.D. Barry, M. Hoorfar, ECS Trans. 58 (2014) 49-58.

[200] D.C. Montgomery, Design and analysis of experiments, Seventh edition, John Wiley \& Sons, 2009.

[201] F. Ettingshausen, J. Kleemann, M. Michel, M. Quintus, H. Fuess, C. Roth, J. Power Sources 194 (2009) 899-907.

[202] S.M. Rezaei Niya, M. Hoorfar, Int. J. Hydrogen Energy 40 (2015) 4868-4873.

[203] S.M. Rezaei Niya, M. Hoorfar, J. Electroanal. Chem. 747 (2015) 112-122. 